\title{
A TECHNIQUE FOR DUAL ADAPTIVE CONTROL
}

\author{
by \\ Jacob Alster, B.Sc., M.Sc. (Technion) \\ Department of Electrical Engineering
}

McGill University,

Montreal, Canada. 


\section{A TECHNIQUE FOR DUAL ADAPTIVE CONTROL}

by

Jacob Alster, B.Sc., M.Sc. (Technion)

A thesis submitted to the Faculty of Graduate Studies and Research in partial fulfilment of the requirements for the degree of Doctor of Philosophy

Department of Electrical Engineering, McGill University, Montreal, Canada.

December, 1972. 


\section{ABSTRACT}

Kalman filtering technique is applied to the problem of multivariable, nonlinear, steady state system identification, and to the problem of real-time adaptive control of linear, stochastic, dynamic systems with random parameters.

The steady state system outputs are modelled as polynomials of the inputs and a Kalman filter is used to estimate the polynomials parameters from the system noisy measurements. The optimal input is synthesized to minimize the estimation error covariance.

A technique for dual adaptive control of dynamic, stochastic systems is developed. For real-time control the system parameters are filtered from noisy measurements. The dual control policy supervises the model identification in addition to system regulation.

The dual controller is evaluated by system simulations and its performance is compared with a non-dual control policy.

In an environment with incomplete knowledge of noise statistics, the dual control law is implemented with an adaptive filtering technique that is estimating sequentially the noise covariance. 


\section{ACKNOWLEDGEMENTS}

I am happy to have this opportunity to express my appreciation to my thesis supervisor Professor P.R. Bélanger for his guidance, helpful suggestions, and positive criticism throughout the course of this research.

I have had many stimulating discussions with my fellow graduate students of the Control Group, which I gratefully acknowledge.

Appreciation is expressed for the financial assistance from the National Research Council and the Department of Electrical Engineering.

Thanks are dive to Miss $P$. Cunningham for her effort and patience in typing the thesis.

My warm thanks are also offered to Dr. and Mrs. D. Ginsburg, whose advice, encouragement, and active support helped in no small way to make this project possible.

I would like to offer special thanks to my parents whose confidence in me and encouragement are indelibly impressed in my memory.

Finally, let me offer my hearffelt thanks to my wife, Jonina, for her patience and understanding during these intensive effort years of my graduate studies, and to my children Sharon and Danny who have missed much weekend fun because "daddy had to study". 
ABSTRACT

$\underline{\text { Page }}$

ACKNOWLEDGEMENTS

TABLE OF CONTENTS

LIST OF SYMBOLS AND ABBREVIATIONS v

CHAPTER I INTRODUCTION I

1.1 The General Problems and Background. 1

1.2 Thesis Contributions and Organization 3

1.2.1 Identification of Steady-State Systems 3

1.2.2 Dual Adaptive Control 6

1.2.3 Control System Simulation 10

1.2.4 Adaptive Control with Adaptive Filtering 11

CHAPTER II OPTIMAL INPUT FOR IDENTIFICATION OF NON-LINEAR SYSTEMS IN STEADY-STATE 14

2.1 Introduction 14

2.2 Problem Formulation $\quad 15$

2.3 Method of Identification 17

2.4 Optimal Input Determination 21

2.4.1 Dynamic N-Stage Optimization 22

2.4.2 Conjugate Gradients Method of N-Stage

2.4.3 One-Stage Minimization 27

2.5 Properties of the Estimator 33

2.6 The Cramér-Rao Lower Bound 42

2.7 System Simulation 46

2.7.1 Example 46

CIJAPTER III . DUAL CONTROL OF DYNAMIC LINEAR STOCHASTIC SYSTEMS

3.1 Introduction 53

3.2 Formulation of the Problem 55

3.3 The Optimal Non-dual Control Law 57

3.4 A Technique for Dual Adaptive Control 66

CHAPTER IV ADAPTIVE CONIROL SYSTEM SIMULATION 80

4.1 Introduction 80

4.2 First-Order System Simulation 81

4.2.1 Example 1. Average Performance Comparison 89

4.2.2 Example 2. Single Run Results. 92 
4.2.3 Example 3. Average Performance with Incomplete Knowledge of Noise Covariance.

4.2.4 Example 4. Single Run Results with Incomplete Knowledge of Noise Covariance

4.2.5 Example 5. Repeated Turn-off Phenomena 103

4.2.6 Example 6. Two Forms of Dual Controller 106

4.2.7 Example 7. Steady State Results 109

4.2.8 Example 8. Steady State Without Exact Knowledge of Noise Covariance 110

4.2.9 Example 9. Deterministic Unknown Parameters i i2

4.2.10 Example 10. Non-Gaussian Parameter Process, Average Performance

4.2.11 Example 11. Non-Gaussian Parameter Process, Single Run Results

4.3 Second Order System Simulation 117

4.3.1 Example 1. Comparison of Average Performance 121

4.3.2 Example 2. Single Run Results 123

CHAPTER $V$

ADAPTIVE CONTROL WITH ADAPTIVE FILTERING 126

5.1 Introduction 126

5.2 Development Of The Adaptive Filter 126

5.3 Dual Control With Adaptive Filtering 132

5.4 System Simulation 135

5.4.1 Example 1. Comparison of Average Performance 137

5.4.2 Example 2. Single Run Results 140

$\begin{array}{lll}\text { CHAPTER VI CONCLUSION } & 145\end{array}$

6.1 Thesis Contributions 145

6.2 Suggestions for Further Research 146

6.3 Final Observations 146

$\begin{array}{ll}\text { REFERENCES } & 147\end{array}$

APPENDIX A $\quad \cdot 152$

$\begin{array}{ll}\text { APPENDIX B } & 155\end{array}$ 


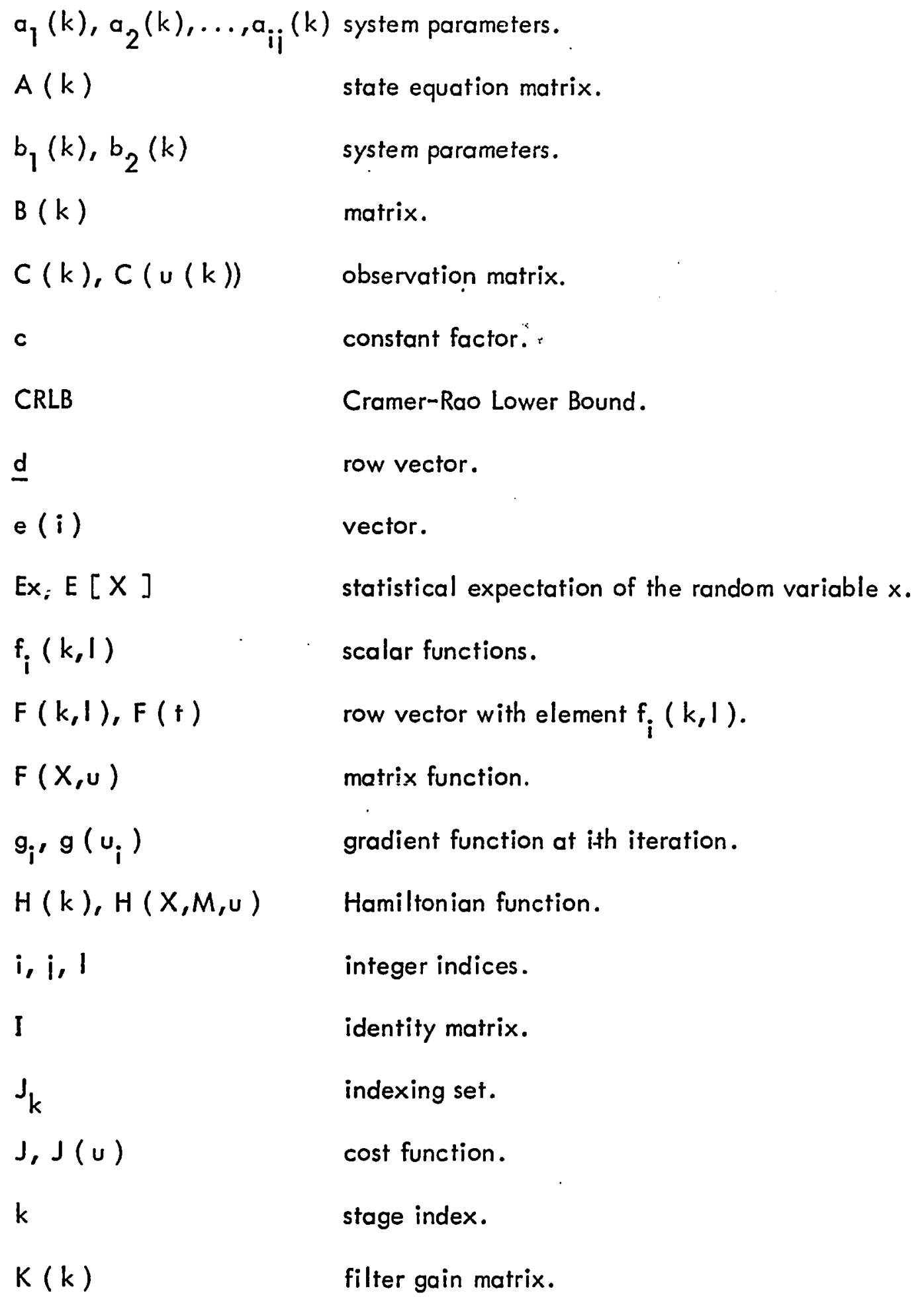




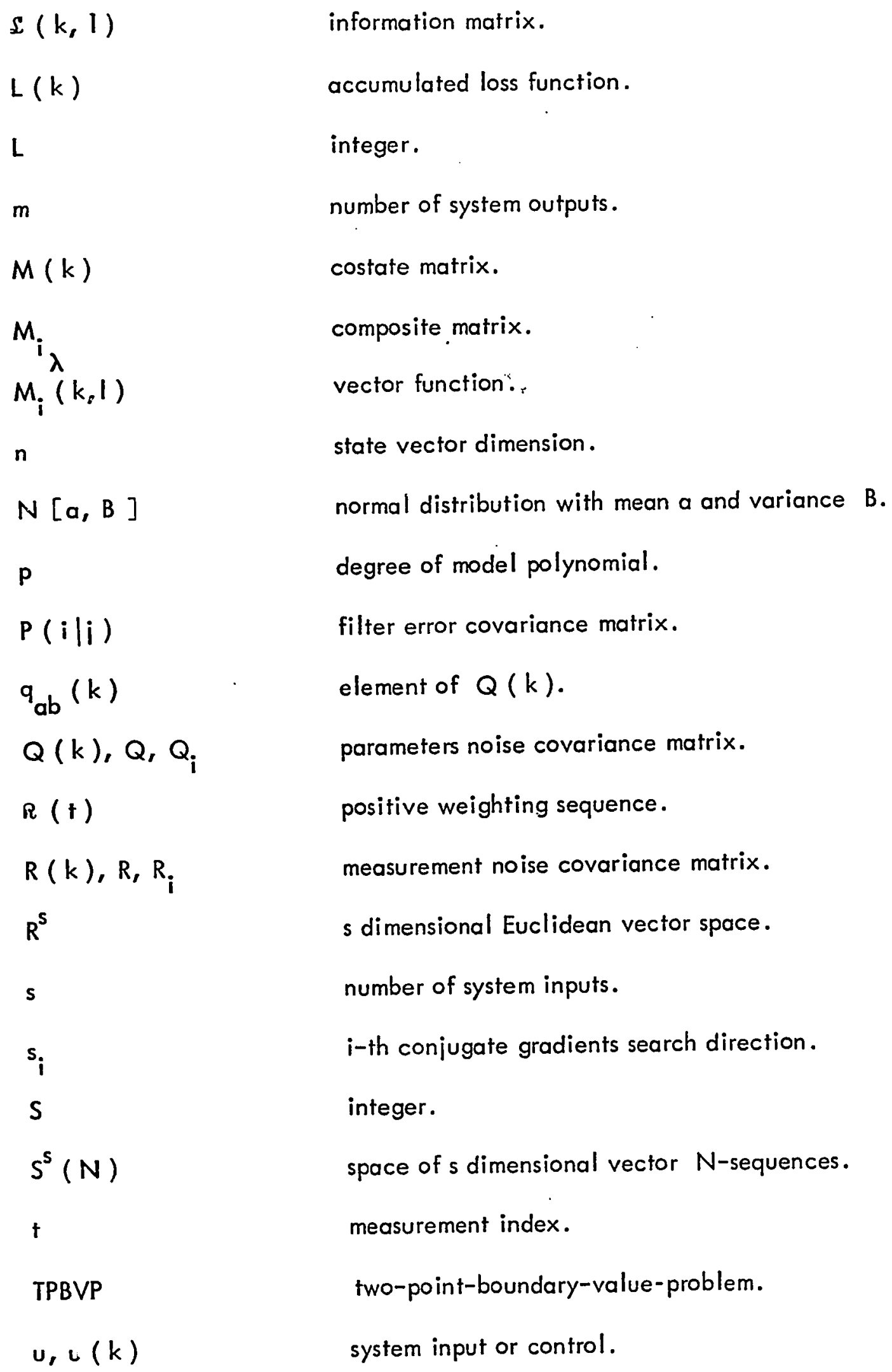




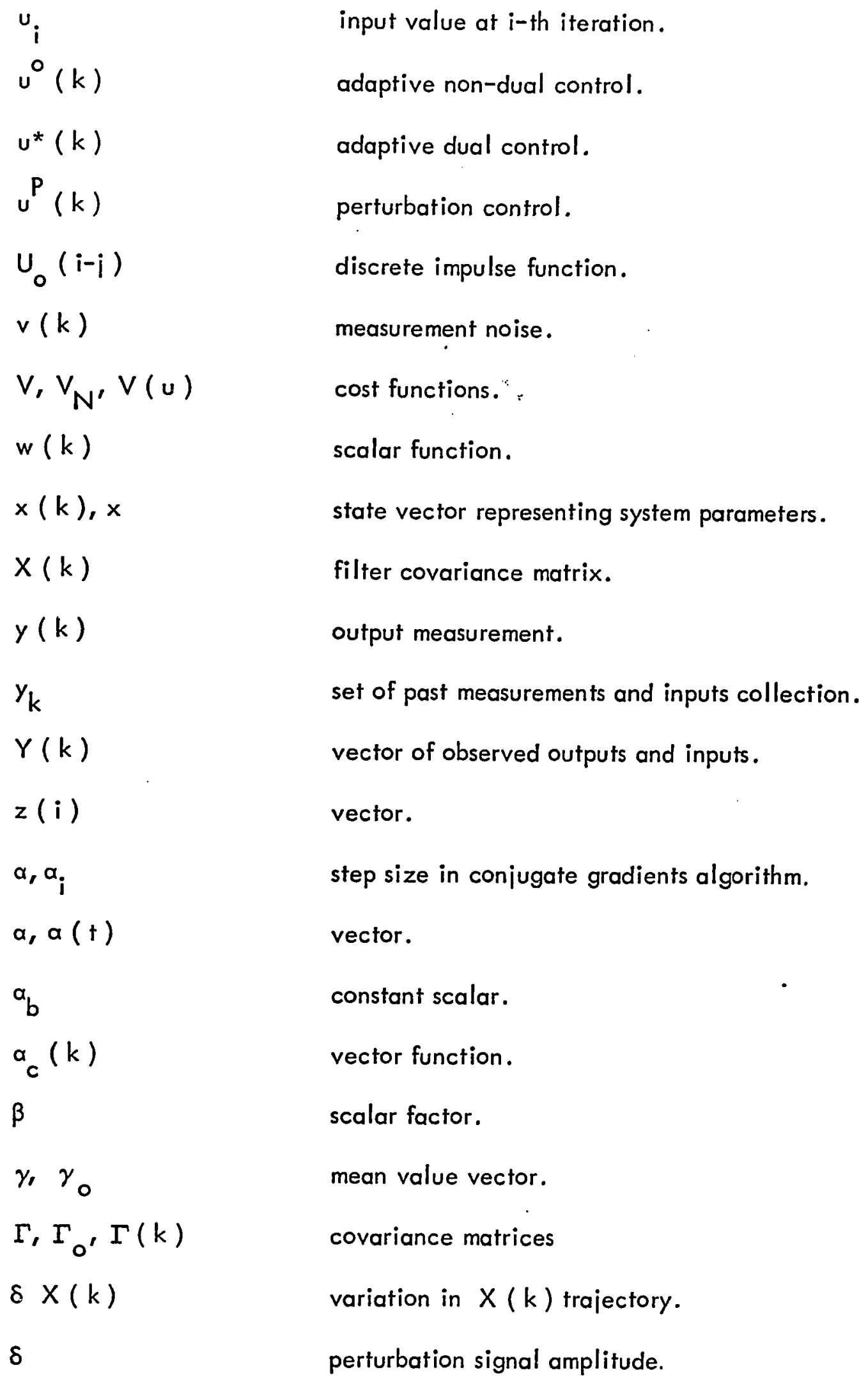




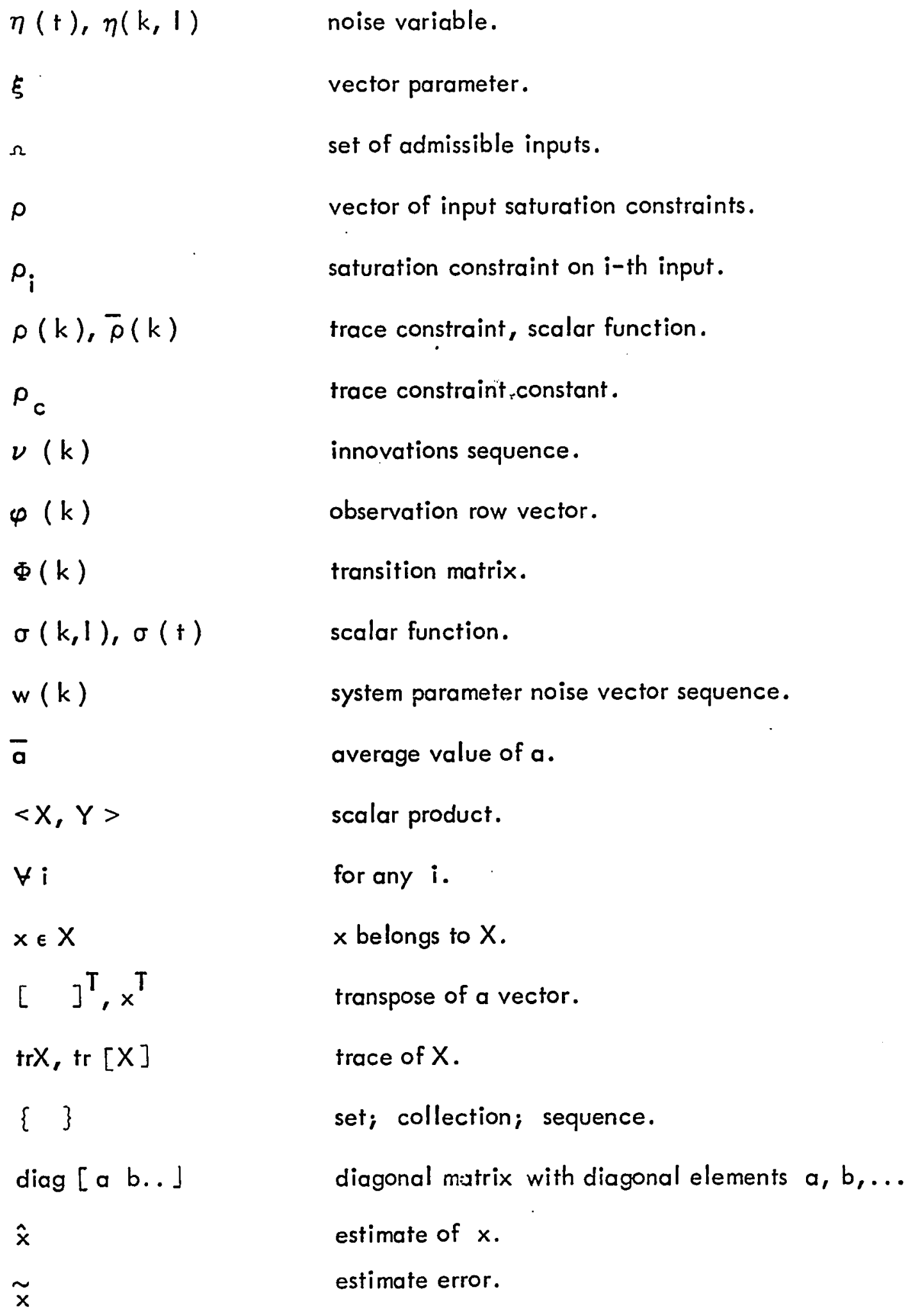




\section{CHAPTER I}

INTRODUCTION

\subsection{The General Problems and Background}

This thesis is basically concerned with the problem of input signal design for identification and control of certain classes of discrete stochastic control systems. The underlying idea is the application of Kalman filtering theory and the optimization of the identification and control processes.

There is a plethora of publications on the art and theory of filtering Kalman's paper [ 11$]$ and Kailath's paper [ 10$]$ give a foundation of the theory for linear discrete systems. References, $[1,2],[13],[35],[43]$ give a reasonably complete survey of various methods and application in the filtering art.

(1) The first class of systems to be dealt with are multivariable nonlinear systems in steady state. Here we are concarned with modelling and identification of the model parameters. The identification is done by estimating the parameters which are modelled as state variables in a dynamic system. The estimator is a Kalman filter and the filter variance diepends on the input signal. Thus, the input is optimized to yield minimum variance of the estimation erro.

The problem of designing input input signals which are optimal from the identification point of view has been discussed, using different approaches in references $[35,36]$ and $[45-47]$. 
The optimal input determinution uses an optimal control problem formulation which uses the Kalman covariance matrix equation as the dynamic equality constraint. This approach has been used by Athans and Schweppe [ 24$]$ in the design of radar waveform, and by Vander Stoep [ 48 ]and Murphy [49 ] .

An exhaustive survey of system identification and process-parameter estimation can be found in the recent survey paper of $\AA$ ström and Eykhoff [50 ]. 2. The second class of systems of interest are linear discrete stochastic dynamic systems with random parameters. We use the input-output difference equation description of such systems as our model. The problem now is the construction of an optimal adaptive control law for the regulation problem. The solution involves problems of identification and control and their interaction. A good treatment of this subject appears in Aström and Wittenmark [3 ].

To obtain real-time control the present system parameters estimates are sequentially produced by a Kalman filter, and on their basis the control strategy is chosen. This pulicy, however, separates the control and identification disregarding the intrinsic mutual dependence of estimation and control. The resulting control law is not dual (Feldbaum [16]) and is shown by Wittenmark and Wieslander $[4,5]$ and here, to be unsatisfactory.

To overcome this difficulty a new problem formulation is used which constrains the estimator error covariance to remain within certain bounds. The solution is a control law of dual nature with superior performance. 
Another approach to achieve duality is the perturbation technique used in $[4,5]$. The problems of identification and control of siochiastic systems modeled by input-output difference equations are treated also in §ström et al. $[1,4,50]$. Other references on the relationships between the problems of identification and control are $[49,51,52]$. Numerous references on many aspects of optimal stochastic control can be found in Wonham [57 ].

3. The problem of adaptive control of stochastic systems is tied with the problem of adaptive filtering, when the controller operates in an environment without complete noise statistics. The dual control law is implemented with an adaptive filter, based on a noise covariance estimator due to Bélanger [9]. Problems of adaptive filtering and other forms of adaptive filters are found in [53-56] .

\subsection{Thesis Contribu' ions and Organization}

In the followin sections we give a description of the main topics and results in the order of their appearance in the thesis.

\subsubsection{Identification of Steady-State Systems}

Chapter 2 develops a method for the determination of optimal inputs for the purpose of identification of multivariable non-linear systems in steady-state. A model is assumed in which the observed output is a polynomial of the inputs and is corrupted by additive discrete white noise. As an example consider the two-inputs one-output model 
$y(k)=x_{1}(k) u_{1}^{2}(k)+x_{2}(k) u_{2}^{2}(k)+x_{3}(k) u_{1}(k)+x_{4}(k) u_{2}(k)+x_{5}(k)+v(k)$

where $v(k) \sim N[0, R(k)]$ and the $x_{i}(k)$ are the unknown parameters to be identified, and are assumed to be stochastic Wiener processes

$$
x(k+1)=x(k)+w(k)
$$

where $x(k)=\left[x_{1}(k) \ldots x_{5}(k) .\right]^{\top}$, and $\omega(k)=\left[\omega_{1}(k) \ldots \omega_{5}(k)^{\top}\right.$ is a white noise random vector sequence $\omega(k) \sim N[0, Q(k)]$. By defining the observation matrix

$$
C(k)=\left[u_{1}^{2}(k) u_{2}^{2}(k) u_{1}(k) u_{2}(k) \quad 1\right]
$$

the model $(1.1)$ is written as

$$
y(k)=C(k) \times(k)+v(k)
$$

The parameters vector $x(k)$ is now identified by applying the Kalman filter technique to the dynamic system (1.2), (1.4).x (k) is estimated as $\hat{x}(n+1)$ using $N$ observations $\{y(1), \ldots, y(N)\}$.

The system model and the Kalman filter used for estimation are described in Sections 2.2 and 2.3. The filter consists of the equations

$$
\begin{aligned}
\hat{x}(k+1) & \hat{x}(k)+K(k)[y(k)-C(k) \hat{x}(k)] \\
K(k)= & X(k) C^{\top}(k)\left[C(k) X(k) C^{\top}(k)+R(k)\right]^{-1} \\
X(k+1)= & X(k)-X(k) C^{\top}(k)\left[C(k) X(k) C^{\top}(k)+R(k)\right]^{-1} \\
& \cdot C(k) X(k)+Q(k)
\end{aligned}
$$


The optimal input determination is done in Section 2.4. First it is noticed that the error covariance matrix $X(k)$ is a function of the input vector $u(k)=\left[\begin{array}{ll}u_{1}(k) & u_{2}(k)\end{array}\right]^{\top}$ as can be seen from Equations (1.7) and (1.3). Thus an optimal control problem is formulated, the solution of which is the optimal input sequence for best identification:

$$
\begin{aligned}
& \text { Minimize } \\
& \{u(k)\} \in \Omega
\end{aligned} \quad \operatorname{tr} X(N+1)
$$

subject to the equality constraint (1.7), with $X(1)$ given; $k=1, \ldots, N$.

The admissible control set $\Omega$ is defined by the saturation constraints $\left|u_{i}(k)\right| \leq \rho_{i}$, $k=1, \ldots, N$.

It is shown that this optimal control problem cannot be solved by the matrix minimum principle (Athans $[23]$ ) since the required convextiy assumption (Halkin [ 18 ]) does not hold. Thus, a matrix conjugate gradient algorithm is developed by which the solution is achieved. The solution essentially consists of solving a Two-Point-Boundary-Value-Problem in the space of $\mathrm{N}$-matrix sequences, and is extremely time consuming.

A simplification is suggested by a procedure of $N$ sequential function minimizations:

$$
\begin{aligned}
& \text { Minimize } \operatorname{tr} X(k+1) \text { given } X(k) \\
& u(k)
\end{aligned}
$$

where

$$
\left|u_{i}(k)\right| \leq \rho_{i}, \text { for } k=1, \ldots, N \text {. }
$$


It is proved that this sequential minimization is optimal, and solves the $\mathrm{N}$-stage optimization problem of (1.8).

Section 2.5 brings forth some properties of the model under the estimation policy, and of the estimation error covariance matrix. In particular the problem of observability and the problem of the bound on the error covariance are discussed.

Section 2.6 deals with the identification problem from the statistical parameter estimation point of view. The covariance matrix of the estimation error of any unbiased estimate of a constant parameter vector is known to be bounded from below by the Cramer-Rao lower bound (CRLB) $[35-40]$. It is shown that the optimal input determined by (1.8) or (1.9) minimizes the trace function of the CRLB. This is equivalent to maximizing the information about the system parameters.

Section 2.7 presents an example of the identification of a two-input two-output system with 8 parameters using the calculated optimal input sequence.

\subsubsection{Dual Adaptive Control}

Chapter 3 develops a technique for dual adaptive control of stochastic dynamic systems, modeled by input-output difference equations with scalar input and output. The output is observed with additive white noise sequence. As an example consider the first order model

$$
y(k)=a(k) y(k-1)+b(k) u(k-1)+v(k)
$$


where $x(k)=[a(k) b(k)]^{\top}$ is assumed a Wiener process

$$
x(k+1)=x(k)+w(k)
$$

and $\omega(k)=\left[\omega_{a}(k) \omega_{b}(k)\right] \sim N[0, Q] ; v(k) \sim N[0, R]$.

The control problem consists of the output regulation, where the controller is designed to keep the output as close to zero as possible. A natural choice of cost function is

$$
V_{N}=E\left[\sum_{k=1}^{N} y^{2}(k)\right]
$$

The minimization of $V_{N}$ yields an optimal control which is also of dual nature (Feldbaum [ 16$]$ ). It serves optimally the purpose of control and identification. Unfortunately the minimization of (1.12) necessitates the solution of a dynamic programing functional equation which cannot be done in real-time, and thus the criterion (1.12) is not of much practical value for real-time control.

In section 3.3 we derive the non-dual adaptive control law $[4]$, by minimizing the single stage cost function:

$$
\begin{aligned}
& \text { Minimize } V=E\left[y^{2}(k+1)\right] \\
& u(k)
\end{aligned}
$$

subject to equations $(1.10),(1.11)$ and where the admissible control is a function of the collection

$$
y_{k}=\{y(k), y(k-1), \ldots, u(k-1), u(k-2), \ldots\}
$$


By using a fundamental Lemma it is shown that (1.13) is equivalent to

$$
\begin{aligned}
& \underset{u(k)}{\operatorname{Minimize} E\left[y^{2}(k+1) \mid y_{k}\right]} \\
& u
\end{aligned}
$$

the solution of which defines a control law. For the system (1.10) this control law is

$$
-u^{\circ}(k)=\frac{\hat{b}(k+1 \mid k)+P_{b b}(k+1 \mid k)}{\hat{b}^{2}(k+1 \mid k) \hat{a}\left(k+l_{r} \mid k\right)+P_{a b}(k+1 \mid k)} y(k)
$$

where

$$
\begin{aligned}
& \hat{x}(k+1 \mid k)=[\hat{a}(k+1 \mid k) \hat{b}(k+1 \mid k)] \\
& P(k+1 \mid K)=\left[\begin{array}{ll}
P_{a a}(k+1 \mid k) & P_{a b}(k+1 \mid k) \\
P_{b b}(k+1 \mid k) & P_{b b}(k+1 \mid k)
\end{array}\right]
\end{aligned}
$$

are the Kalman estimate and error covariance matrix, generated by the filter

$$
\begin{aligned}
& \hat{x}(k+1 \mid k)=\hat{x}(k \mid k-1)+K(k)[y(k)-\varphi(k-1) \hat{x}(k \mid k-1)] \\
& K(k)=P(k \mid k-1) \varphi^{\top}(k-1)\left[\varphi(k-1) P(k \mid k-1) \varphi^{\top}(k-1)+R\right]^{-1} \\
& P(k+1 \mid k)=P(k \mid k-1)-K(k) \varphi(k-1) P(k \mid k-1)
\end{aligned}
$$

where

$$
\varphi(k-1)=[y(k-1) \quad u(k-1)] .
$$

The control law is not dual and fails to be satisfactory if the estimation is bad. It is not capable of improving the identification, and thus is adversely affected by two kinds of phenomena: the turn-off phenomenon and the burst phenomenon. The 
turn-off phenomenon produces almost zero control signals and leaves the system with virtually no control for long periods of time. The burst phenomenon erroneously produces very large control signals, which result in large oscillating output values.

In section 3.4 we develop a dual control policy. Motivated by the idea that the controller should supervise the estimator, as well as the system control, we design a controller that observes a measure of the estimation error variance, and controls its magnitude. The following relation is shown to exist

$$
P^{-1}(k+1 \mid k+1)=P^{-1}(k+1 \mid k)+\varphi^{\top}(k) R^{-1} \varphi(k)
$$

which for $(1.10)$ becomes after taking the trace function

$$
\operatorname{trP} P^{-1}(k+1 \mid k+1)=\operatorname{trP} P^{-1}(k+1 \mid k)+\frac{1}{R}\left[y^{2}(k)+u^{2}(k)\right]
$$

a quadratic function of the control $u(k)$. This simple dependence of the measure of information on $u(k)$ is exploited in the dual control problem formulation:

$$
\begin{aligned}
& \operatorname{Minimize} E\left[y^{2}(k+1) \mid y_{k}\right] \\
& u(k)
\end{aligned}
$$

subject to the information constraint

$$
\rho(k+1)=\operatorname{trP}^{-1}(k+1 \mid k+1) \geq \bar{\rho}(k+1)
$$

where $\bar{\rho}(k+1)$ is a predetermined function.

The solution for system (1.10) is the dual control $u^{*}(k)$ policy, 


$$
\begin{gathered}
u^{*}(k)= \begin{cases}\bar{u}(k) \operatorname{sgn} u^{0}(k) & \text { if }\left|u^{0}(k)\right| \leq \bar{u}(k) \\
u^{0}(k) & \text { otherwise }\end{cases} \\
\bar{u}(k)= \begin{cases}\sqrt{\mu(k)} & \text { if } \mu(k)>0 \\
0 & \text { otherwise }\end{cases} \\
\mu(k)=R\left[\bar{\rho}(k+1)-\operatorname{trP}^{-1}(k+1 \mid k)\right]-y^{2}(k)
\end{gathered}
$$

and $u^{\circ}(k)$ is given by (1.15).

Under the application of the dual control law the estimation of the system parameters is shown to be invariant under the system (observation) noise intensity.

\subsubsection{Control System Simulation}

Chapter 4 contains several examples of system simulation. The dual controller is compared with the non-dual one, and with the perturbation controller (Wieslander and Wittenmark $[4,5]$ ) which is obtained by adding a small perturbation square wave signal to the non-dual controller for the purpose of improving the identification

$$
u^{P}(k)=u^{0}(k)+(-1)^{k} \cdot \delta
$$

where $\delta^{\prime}$ is the square-wave amplitude.

In Section 4.2 the controllers are compared for a first order system with two parameters as given by (1.10). The comparison is done under different noise conditions 
and different random parameter processes, where the average as well as simple runs performances are compared. The implementations of the dual controller is discussed and a simple case where the information measure $\bar{\rho}(k+1)$ is taken to be constant is compared with an improved version where $\bar{\rho}(k+1)$ is time varying.

It is found that the dual and perturbation controllers are superior to the non-dual control policy, and are generally equal when the parameters noise statistics are exactly known. In the case of incomplete information about $Q$, numerical experiments show a certain advantage of the dual controller over the perturbation control policy.

Section 4.3 contains two examples of a second order system with three random parameters

$$
y(k)=a_{1}(k) y(k-1)+a_{2}(k) y(k-2)+b(k) u(k-1)+v(k)(1.26)
$$

The three controllers performance is again compared.

\subsubsection{Adaptive Control With Adaptive Filtering}

The adaptive dual control law (1.22) - (1.24) uses the Kalman filter estimates and error covariance matrix. For optimal generation of these variables the noise covariances $Q$ and $R$ are needed. In the absence of their knowledge there exist methods of estimating them. One method is that of Bélanger $[9]$, which uses a least squares filter for sequential estimation of $Q$ and $R$ based on the system observation. Chapter 5 uses this estimator to construct an adaptive filter, using the 
filtered values for $Q$ arid $R$ sequentially in a Kalman filter which estimates the system random parameters.

The covariances $Q$ and $R$ are represented in the form of a linear

combination

$$
\begin{aligned}
& R=\sum_{i=1}^{N} a_{i} R_{i} \\
& Q=\sum_{i=1}^{N} a_{i} Q_{i}
\end{aligned}
$$

where $R_{i}$ and $Q_{i}$ are known. The problem is to best estimate $\alpha=\left[\alpha_{1} \alpha_{2} \ldots \alpha_{N}\right]^{\top}$. The adaptive filter is constructed as

$$
\begin{aligned}
\hat{x}(k+1 \mid k)=\hat{x}(k \mid k-1)+K(k)[y(k)-\varphi(k-1) \hat{x}(k \mid k-1)] & \begin{aligned}
K(k) & =P(k \mid k-1) \varphi^{\top}(k-1)\left[\varphi(k-1) \rho(k \mid k-1) \varphi^{\top}(k-1)\right. \\
& +\hat{R}(k)]^{-1}
\end{aligned} \\
P(k+1 \mid k) & =P(k \mid k-1)-K(k) \varphi(k-1) \rho(k \mid k-1)+\hat{Q}(k)
\end{aligned}
$$

where $\hat{R}(k)$ and $\hat{Q}(k)$ are the estimates of $R$ and $Q$ at stage $k$, and are obtained by using the estimated vector $\hat{a}(k)$ in (1.27) and (1.28),

$$
\hat{R}(k)=\sum_{i=1}^{N} \hat{a}_{i}(k) R_{i} ; \quad \hat{Q}(k)=\sum_{i=1}^{N} \hat{a}_{i}(k) Q_{i}
$$

The estimates $\hat{a}(k)$ are obtained by using Bélanger's algorithm which is founded on the idea that the innovations sequence 


$$
\nu(k)=y(k)-\varphi(k-1) \hat{x}(k \mid k-1)
$$

should be made white. The algorithm is given in Section 5.2

Section 5.3 describes the controller which combines the dual control law and the adaptive filter.

In section 5.4 a first order system with two random parameter is simulated. The noise covariance matrices $\underset{r}{Q}$ and $R$ are assumed unknown and the average performance of four control policies is compared:

(1) non-dual control with non-adaptive filtering.

(2) dual control with non-adaptive filtering.

(3) non-dual control with adaptive filtering.

(4) dual control with adaptive filtering.

The superiority of the fourth case of dual control with adaptive filtering is clearly demonstrated.

The four control policies are also compared for a single run, and results of the covariances estimation are shown for the dual controller with adaptive filter strategy. 
CHAPTER ॥

OPTIMAL INPUT FOR IDENTIFICATION OF

NON-LINEAR SYSTEMIS IN STEADY-STATE

\subsection{Introduction}

In this chapter we aim at developing a method for optimal input design. The purpose of this input signal is to facilitate optimal identification of multivariable, non-linear models of systems in steady-state.

The system outputs are modeled as polynomials of the inputs, and with this structure, the polynomial coefficients are to be identified.

The unknown parameters are assumed constant, or slowly time varying, and their identification is to be achieved by an estimate, based on $N$ measurements of the system output vector. The identification strongly depends on the input vector sequence of length $N$, as the measurement is a function of the inputs.

At each stage a certain input vector is applied, and a measurement of the system output vector is taken, after the steady-state is reached. Hence, this is a discrete process. The output measurement is assumed to include an additive white noise vector.

The unknown parameters are assumed to be states in a discrete dynamical linear system driven by white noise. This state vector is to be identified. This representation, fogether with the observation equation, is amenable to the 
application of Kalman filtering theory.

The parameters vector is estimated by a Kalman filter, and the dependence of the estimation error covariance matrix on the input is shown.

The identification problem is formulated as an optimal control problem, involving matrix equations, and a solution is derived using the conjugate gradient method in a dynamic, $\mathrm{N}$-stage, trajectory optimization.

The resulting Two-Point-Boundary-Value-Problem solution is then simplified by a single-stage function optimization procedure. The two solutions are shown to be identical.

Some properties of the estimator are investigated and the optimal input is shown to minimize the Cramer-Rao lower bound on the error covariance matrix, thus demonstrating the optimality of the identification policy.

\subsection{Problem Formulation}

We are given a discrete measurement model in the form of input-output relation

$$
\begin{aligned}
& y_{i}(k)=a_{i, 0,0}(k)+\sum_{i=1}^{p} \sum_{i=1}^{s} a_{i, i, l}(k) u_{i}^{i}+v_{i}(k) \\
& i=1,2, \ldots, m \quad ; k=1,2, \ldots
\end{aligned}
$$

where

$$
\begin{aligned}
& y_{i}(k) \text { is the } i \text {-th ouput at stage } k \\
& u_{l}^{\beta}(k) \text { is the } 1 \text {-th input (control at stage } k \text {, raised to power } \beta
\end{aligned}
$$


$v_{i}(k)$ is white, gaussian, independent random sequence, with zero mean and covariance

$$
\begin{aligned}
& \operatorname{cov}\left[v_{i}(k) ; v_{i}(a)\right]=r_{i i}(k) U_{0}(k-q) \\
& U_{0}(k-q)= \begin{cases}1 & k=q \\
0 & k \neq q\end{cases}
\end{aligned}
$$

and it represents the measurement noise.

$$
\begin{aligned}
& a_{i j, l}(k) \text { is an unknown system parameter } \\
& m, s, p \text { are given integers representing the number of outputs, } \\
& \text { inputs and highest power of the input respectively. }
\end{aligned}
$$

An example of such a system is one with two outputs (measurements) and two inputs (controls) which are represented as second order polynomials

$$
\begin{aligned}
y_{1}(k)= & a_{1,0,0}(k)+a_{1,1,1}(k) u_{1}(k)+a_{1,1,2}(k) u_{2}(k)+a_{1,2,1}(k) u_{1}^{2}(k) \\
& +a_{1,2,2}(k) u_{2}^{2}(k)+v_{1}(k) \\
y_{2}(k)= & a_{2,0,0}(k)+a_{2,1,1}(k) u_{1}(k)+a_{2,1,2}(k) u_{2}(k)+a_{2,2,1}(k) u_{1}^{2}(k) \\
& +a_{2,2,2}(k) u_{2}^{2}(k)+v_{2}(k)
\end{aligned}
$$

The $m(p+l) s$ model parameters are unknown. They are assumed either constants or time varying, and it is required to identify them at stage $N+1$. The identification is to be done by estimating their values at stage $N+1$, based on 
the noisy measurements $y_{i}(k), i=1, \ldots, m ; k=1,2, \ldots, N$. Note that each measurement here consists of $m$ simultaneous observations at the $m$ outputs.

The measurement at stage $k$ is controlled by the $s$ input signals $u_{1}(k) \quad 1=1, \ldots, s$, through the algebraic input-output relation of Equation (2.1). Hence by selecting different control policies we get different measurement sequences, and this leads to the following problem:

Choose the optimal input sequence from an admissible set, that results in the best identification of the model parameters.

This general problem will be made precise below, after discussing the method used for identification.

\subsection{Method of Identification}

The system parameters are identified, using Kalman's filtering technique [11]. They are represented as states in a dynamical system, and are estimated using a discrete Kalman filter.

For this purpose we arrange the parameters in vector form

$$
x=\left[\begin{array}{llll}
\alpha_{1}^{\top} & \alpha_{2}^{\top} & \cdots & \alpha_{m}^{\top}
\end{array}\right]^{\top}
$$

where $a_{i}$ is a vector of the form 


$$
\alpha_{i}=\left[\begin{array}{llll}
\alpha_{i 1}^{\top} & \alpha_{i 2}^{\top} & \ldots & \alpha_{i P}^{\top}
\end{array}\right]^{\top} ; i=1, \ldots, m
$$

and $a_{i j}$ is again a vector

$$
a_{i i}=\left[a_{i, 0,0} a_{i, i, 1} a_{i, i, 2} \cdots a_{i, i, s}\right] ; i=1, \ldots, p
$$

Thus the parameters are arranged now in a certain order, and we may use either one of two assumptions. In the first instance we assume the parameters vector to be a constant, and thus we write

$$
x(k+1)=x(k)
$$

or alternatively assume $x(k)$ to be a random vector generated by the Wiener stochastic process

$$
x(k+1)=x(k)+\omega(k)
$$

where

$$
\omega(k) \sim N[0, Q(k)]
$$

is a sequence of normal, independent random vectors, with zero mean and covariance matrix $Q(k) . x(k)$ and $\omega(k)$ are of dimension $n$, and $n=m\left(p^{+1}\right) s$.

The observation Equations (2.1) can be written in vector form as

$$
y(k)=C(k) \times(k)+v(k)
$$


where $y(k)$ and $v(k)$ are $m$ dimensional vectors

$$
\begin{aligned}
& y(k)=\left[y_{1}(k) y_{2}(k) \ldots y_{m}(k)\right]^{\top} \\
& v(k)=\left[v_{1}(k) v_{2}(k) \ldots v_{n}(k)\right]^{\top} \\
& v(k) \sim N[0, R(k)]
\end{aligned}
$$

where $R(k)$ is the covariance matrix of the white, gaussian, vector sequence $v(k)$, with elements $\tau_{i j}(k)$ as in Equation (2.2).

$C(k)=C(u(k))$ is an $m \times n$ matrix function of the input vector $u(k)$, where

$$
u(k)=\left[u_{1}(k) u_{2}(k) \ldots u_{s}(k)\right]
$$

and is of the form

$$
C=\left[\begin{array}{ccccc}
\underline{d} & \underline{0} & \cdot & \cdot & \underline{0} \\
\underline{0} & \underline{d} & \cdot & \cdot & \underline{0} \\
\cdot & \cdot & \cdot & \cdot & \cdot \\
\cdot & \cdot & \cdot & \underline{d} & \underline{0} \\
\underline{0} & \underline{0} & \cdot & 0 & \underline{d}
\end{array}\right]
$$

where $\underline{d}$ and $\underline{0}$ are row vectors of dimension $s(p+1), \underline{0}$ has zero entries and

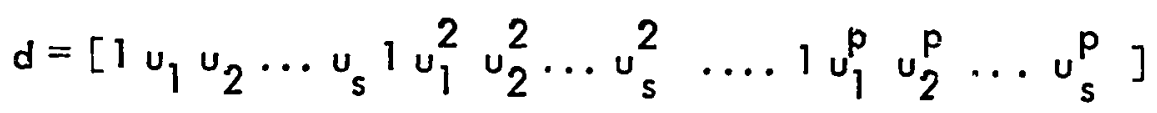

The Equations (2.7), (2.9) represent a discrete linear dynamic system driven by white noise, and with noisy observations, to which the Kalman filter theory can be applied [1], [2]. 
Define the observations sequence

$$
Y_{k}=\{y(1), y(2), \ldots, y(k)\}
$$

The state estimate is the conditional mean

$$
\hat{x}(k \mid i)=E\left\{x(k) \mid Y_{i}\right\}
$$

The estimation error is

$$
\tilde{x}(k \mid i)=x(k)-\hat{x}(k \mid i)
$$

and the error covariance matrix is defined as

$$
P(k \mid i)=\operatorname{cov}[\tilde{x}(k \mid i), \tilde{x}(k \mid i)]
$$

The filter consists of the following equations. Between observations

$$
\begin{aligned}
& \hat{x}(k+1 \mid k)=\hat{x}(k \mid k) \\
& P(k+1 \mid k)=P(k \mid k)+Q(k)
\end{aligned}
$$

At observations

$$
\begin{aligned}
& \hat{x}(k \mid k)=\hat{x}(k \mid k-1)+K(k)[y(k)-C(k) \hat{x}(k \mid k-1)] \\
& P(k \mid k)=P(k \mid k-1)-K(k) C(k) P(k \mid k-1)
\end{aligned}
$$

with the gain $K(k)$ given by

$$
K(k)=P(k \mid k-1) C^{\top}(k)\left[C(k) P(k \mid k-1) C^{\top}(k)+R(k)\right]^{-1}
$$


For simplification, we define

$$
\begin{aligned}
& \hat{x}(k)=\hat{x}(k \mid k-1) \\
& P(k)=P(k \mid k) \\
& X(k)=P(k \mid k-1)
\end{aligned}
$$

and using these variables the filter equations become

$$
\begin{aligned}
\hat{x}(k+1)= & x(k)+k(k) \stackrel{r}{[} y(k)-C(k) \hat{x}(k)] \\
x(k+1)= & x(k)-x(k) C^{\top}(k)\left[C(k) x(k) C^{\top}(k)+R(k)\right]^{-1} \\
& \cdot C(k) X(k)+Q(k)
\end{aligned}
$$

The last equation describes the sequential update of the error covariance matrix of the estimate at stage $k$, based on observations up to stage $k-1$.

The filter consisting of Equations (2.28), (2.29) with the gain

$$
K(k)=X(k) C^{\top}(k)\left[C(k) X(k) C^{\top}(k)+R(k)\right]^{-1}
$$

and the initial estimate and error covariance matrix $\hat{x}(1)$ and $X(1)$, represent the identification method.

\subsection{Optimal Input Determination}

The estimation error variance time-evolution is described by Equation (2.29). It is seen that it depends on the input vector $u(k)$, since $C(k)=C(u(k))$ is a function of the input signal. Thus it is clear that the choice of the input sequence 
$\{u(k)\}$ directly influences the identification. To achieve the best identification using the abovementioned filter, we shall look for an input sequence that will min imize the estimation error. For this purpose we define a cost function and minimize it over all admissible control sequences. A suitable cost function is one which is a non-negative functional of the estimation error. A natural choice is the trace of the error covariance matrix $\operatorname{tr} X(k)$, which is non-negative for initial error covariance matrix, which is positive semi-definite.

\subsubsection{Dynamic N-Stage Optimization}

The discussion above leads naturally to the optimal control problem

formulation:

$$
\text { Minimize } J(u)=\operatorname{tr} X(N+1)
$$

over all admissible control sequences $\{u(k)\} \in \Omega$, where

$$
\Omega=\{\{u(1), u(2), \ldots, u(N)\}:|u(k)| \leq \rho\}
$$

subject to the equality constraint $(2.29)$ with given initial condition $X(1)$. $\rho$ is the vector of control magnitude constraints

$$
\rho=\left[\rho_{1}, \dot{p}_{2}, \ldots, \rho_{s}\right]^{\top}
$$

and the control vector absolute value is in the sense of its components

$$
|u(k)|=\left[\left|u_{1}(k)\right|\left|u_{2}(k)\right| \ldots\left|u_{s}(k)\right|\right]^{\top}
$$


This optimal control problem seems amenable to a solution by the matrix minimum principle as formulated by Athans [23]. This however is not the case, as the needed assumptions of convexity (Halkin [ 18$]$, or directional convexity (Holtzman [19]) do not hold. This is shown in Appendix A.

The dynamic $\mathrm{N}$-stage optimization problem is thus solved using a programming approach, employing the conjugate gradients method.

\subsubsection{Conjugate Gradients Method of N-Stage Minimization}

We use now concepts of variational theory to derive the conjugate gradient matrix functional form. Rewrite the dynamic equality constraint (2.25) in the form.

$$
X(k+1)=F(X(k), u(k))
$$

and adjoin it to the cost function (2.31) [23], [24]

$J(u)=\operatorname{tr} X(N+1)+\sum_{k=1}^{N} \operatorname{tr}[F(X(k), u(k))-X(k+1)] M^{\top}(k+1)$

where $M(k)$ is the costate matrix. Next define the Hamiltonian function

$H(k)=H[X(k), M(k+1), u(k)]=\operatorname{tr} F(x(k), u(k)) M^{\top}(k+1)$

substitute this in (2.36)

$$
J(u)=\operatorname{tr} X(N+1)+\sum_{k=1}^{N} H(k)-\operatorname{tr} X(k+1) M^{\top}(k+1)
$$


The first variation in $J(u)$ under control and state pertubation

is

$$
\begin{aligned}
\delta J(u)= & \operatorname{tr} \delta X(N+1)+\operatorname{tr} \sum_{k=1}^{V} \frac{\partial H(k)}{\partial X(k)} \delta X^{\top}(k)+\frac{\partial H(k)}{\partial u(k)} \delta u^{\top}(k) \\
& -M(k+1) \delta X^{\top}(k+1)
\end{aligned}
$$

The concept of gradient matrix of the form $\frac{\partial f(X)}{\partial X}$ where $f(X)$ is a scalar function of the matrix $X$, and the inner product $\langle X, Y\rangle=\operatorname{tr}\left[X Y^{\top}\right]$ is found in Athens [23], and an extensive treatment appears in [32]. Now,

$$
\begin{aligned}
-\sum_{k=1}^{N} M(k+1) \delta x(k+1) & =-\sum_{k=1}^{N+1} M(k) \delta x^{\top}(k)+M(1) \delta x^{\top}(1) \\
& =-\sum_{k=1}^{N} M(k) \delta x^{\top}(k)-M(N+1) \delta x^{\top}(N+1) \\
& +M(1) \delta x^{\top}(1)
\end{aligned}
$$

Substitute this expression, with $\delta X^{\top}(1)=0$, back into (2.39)

$$
\begin{aligned}
\delta J(u)= & \operatorname{tr}[I-M(N+1)] \delta X(N+1)+\operatorname{tr} \sum_{k=1}^{N}\left\{\frac{\partial H(k)}{\partial X(k)}-M(k)\right] \delta X^{\top}(k) \\
& \left.+\frac{\partial H(k)}{\partial u(k)} \delta u^{\top}(k)\right\}
\end{aligned}
$$

To minimize the first variation in $J(u)$ set

$$
M(k)=\frac{\partial H(k)}{\partial X(k)} \quad ; \quad k=1, \ldots, N
$$


with boundary (terminal) condition

$$
M(N+1)=I
$$

The first variation in the cost function becomes

$$
\delta J(u)=\sum_{k=1}^{N}\left[\frac{\partial H(k)}{\partial u(k)}\right] \delta u^{T}(k)
$$

where $\frac{\partial H(k)}{\partial u(k)}$ is the gradient of $J(u)$ with respect to $u(k)$, while Equations (2.35) and (2.41) are satisfied.

$\delta \mathrm{J}(\mathrm{u})$ is now minimized by choosing (the gradient method)

$$
\delta u(k)=-\epsilon \frac{\partial H(k)}{\partial u(k)}
$$

where $\epsilon$ is a positive number. The conjugate gradients method is more efficient and has better convergence properties $[25]-[23]$. The algorithm used is based on the one described by Lasdon et al. [25 ], with modifications to allow for bounded controls, as suggested by Pagurek and Woodside [27 ],

Define:

$u_{i}=\left\{u_{i}(1), \ldots, u_{i}(N)\right\}-$ the control sequence at the ith iteration $J\left(u_{i}\right)$ - cost function value corresponding to $u_{i}$

$\left.g_{i}=\left\{g_{i}(1), \ldots, g_{i}(N)\right\}=\left\{\frac{\partial H_{i}(1)}{\partial u_{i}(1)}\right], \ldots, \frac{\partial H_{i}(N)}{\partial u_{i}(N)}\right\}$ - the gradient trajectory (sequence) corresponding to $u_{i}$ 
$s_{i}$ - the ith search direction

$\alpha, \alpha_{i}, \beta_{i}$-scalars

The Conjugate Gradients Algorithm

Chosen initially are $u_{0}$ and

$$
\begin{aligned}
& g_{0}=g\left(u_{0}\right) . \\
& s_{0}=-g_{0},
\end{aligned}
$$

then iteratively

$$
\begin{aligned}
& a_{i}=\alpha \text { to minimize } J\left(u_{i}+a_{i}\right) \\
& s_{i+1}=u_{i}+a_{i} s_{i} \\
& g_{i+1}=g\left(u_{i+1}\right) \\
& \beta_{i}=\frac{\left\langle g_{i+1}, g_{i+1}\right\rangle}{\left\langle g_{i}, g_{i}\right\rangle} \\
& s_{i+1}=-g_{i+1}+\beta_{i} s_{i}
\end{aligned}
$$

where $\left\langle g_{i}, g_{i}\right\rangle$ is the inner product, defined by

$$
\left.<g_{i}, g_{i}\right\rangle=\sum_{k=1}^{N} g_{i}^{\top}(k) g_{i}(k)
$$

At each iteration a one-dimensional search for $\alpha_{i}$ must be carried out. This requires repeated evaluations of the cost function and thus is very 
time consuming. An efficient method for this linear search, is one which requires a minimum number of cost function evaluations, with good convergence rate. A quadratic interpolation technique was found to yield satisfactory resilts.

At each iteration the elements of the new control vector are truncated at lower and upper bounds, using the method of Pagurek and Woodside [27]. The elements of $g_{i}$ are considered to be zero when the corresponding control elements saturate, and are used as such while performing the inner product summation. That is, over the interval in which the $i$-th element of $u_{i}$ saturates, the $i$-th element of $g_{i}$ is taken to be zero.

At each iteration the evaluation of $g_{i}$ requires the evaluation of $H\left(u_{i}\right)$, which is done by forward "integration" of the state equation (2.35), up to stage $N+1$, with initial condition $X(1)$, and then "integrating" backwards. equation (2.41) with the initial condition (2.42). The evaluation of the gradient vector follows, $g_{i}=\frac{\partial H\left(u_{i}\right)}{\partial u_{i}}$

\subsubsection{One-Stage Minimization}

The $N$-stage dynamic optimization method, described above solves a Two-Point-Boundary-Value-Problem (TPBVP) in order to minimize the trace of the Kalman filter error variance, at stage $\mathrm{N}+1$. The solution of this TPBVP requires the trajectories computation of matrix equations and is computationally very cumbersome, as well as extensively time consuming.

A much simpler approach is the minimization of the error variance 
at stage $\mathrm{N}+1$ by sequentially minimizing it at each stage. The optimal stage input is obtained, that minimizes the error variance trace function. The problem is thus reduced to a procedure of repeated function minimizations in the $R^{S}$ space, as compared to the dynamic minimization in the space $S^{S}(N)$ - of s dimensional vector sequences of length $\mathrm{N}$.

The one-stage optimization procedure seems as a suboptimal solution to the problem of

$$
\begin{aligned}
& \min _{u(1), \ldots, u(N)} \operatorname{tr} X(N+1)
\end{aligned}
$$

but as we shall prove later, it is identical to the $\mathrm{N}$-stage dynamic optimization.

One-Stage Optimization Procedure:

$$
\text { Minimize } J_{1}(u(k))=\operatorname{tr} X(k+1) ; k=1,2, \ldots, N
$$

over all admissible control vectors $u(k) \in \Omega$,

$$
\Omega_{1}=\left\{u(k):\left|u_{i}(k)\right| \leq \rho_{i}, i=1,2, \ldots, s\right\}
$$

where

$$
\begin{aligned}
J_{1}(u(k))= & \operatorname{tr} X(k)-\operatorname{tr}\left\{X ( k ) C ^ { \top } ( u ( k ) ) \left[C(u(k)) X(k) C^{\top}(u(k))\right.\right. \\
& \left.+R(k)]^{-1} C(u(k)) X(k)\right\}
\end{aligned}
$$

with given initial $\times(1)$.

This is a sequence of identical function minimizations over a closed 
bounded set in the real Euclidean space $R^{5}$.

An efficient numerical method of minimization is again the conjugate gradients procedure which is summarized below for function minimization [24], [30].

Define:

$u_{i}$ the minimizing arguement at the $i-$ th iteration,

$J_{j}\left(u_{i}\right)$ the function value corresponding to $u_{i}$,

$g_{i}=\left.\frac{\partial J_{1}(u)}{\partial u}\right|_{u=u_{i}}$ the gradient vector corresponding to $u_{i}$.

Choose initially $u_{0}$ and evaluate $g_{0}$

$$
s_{0}=-g_{0}
$$

Repeat at each iteration

$$
\begin{aligned}
& \alpha_{i}=\alpha \text { to minimize } J\left(u_{i}+\alpha s_{i}\right) \\
& u_{i+1}=u_{i}+\alpha_{i} s_{i} \\
& g_{i+1}=g\left(u_{i+1}\right) \\
& \beta_{i}=\frac{g_{i+1}^{T} g_{i+1}}{g_{i}^{T} g_{i}} \\
& s_{i+1}=-g_{i+1}+\beta_{i} s_{i}
\end{aligned}
$$

The elements of the minimizing argument $u_{i}$ are truncated at their saturation levels, and the corresponding elements of $g_{i}$ are set to zero in the 
computation of $\beta_{i}$, as mentioned in Section 2.4.2.

in the sequel we make use of an important matrix identity.

Lemma 2.1

Let $X, R$ and $C$ be $n \times n, m \times m$ and $m \times n$ matrices, respectively. Suppose that $X$ is positive definite and $R$ positive definite. Then

$$
\left[X^{-1}+C^{\top} R^{-1} C\right]^{-1}=x-X C^{\top}\left[C X \cdot C^{\top}+R\right]^{-1} C X
$$

Proof:

See e.g. [2], appendix 7B.

We are now prepared to prove that the sequential one-stage optimization solution is optimal.

Theorem 2.1

For the model $(2.6),(2.9)$

$$
\begin{aligned}
& x(k+1)=x(k) \\
& y(k)=C(u(k)) x(k)+v(k)
\end{aligned}
$$

and the $\mathrm{N}$-stage optimization problem (2.31) - (2.34), the one-stage optimization procedure $(2.53)-(2.55)$, is optimal.

Proof:

Using lemma 2.1, the state equation (2.29) can be written as 


$$
X^{-1}(k+1)=X^{-1}(k)+C^{\top}(u(k)) R^{-1}(k) C(u(k))
$$

since $Q(k)=0$, and from the Kalman filtering theory it follows that $X(k)$ is positive definite if $R(k)$ and $X(1)$ are positive definite. Thus we can write

$$
\begin{aligned}
X^{-1}(N+1) & =X^{-1}(N)+C^{\top}(u(N)) R^{-1}(N) C(u(N)) \\
& =X^{-1}(N-1)+C^{\top}(u(N-1)) R^{-1}(N-1) C(u(N-1) \\
& +C^{\top}(u(N)) R^{-1}(N) C(u(N)) \\
& \vdots \\
= & X^{-1}(1)+\sum_{i=1}^{N} C^{\top}(u(i)) R^{-1}(i) C(u(i))
\end{aligned}
$$

Hence the cost function (2.31) can be written as

$$
J(u)=\operatorname{tr} X(N+1)=\operatorname{tr}\left[X^{-1}(1)+\sum_{i=1}^{N} C^{\top}(u(i)) R^{-1}(i) C(u(i))\right]^{-1}
$$

The $N$-stage minimization calls for the minimization of $\mathrm{J}(\mathrm{u}(\mathrm{l})$, $u(2), \ldots, u(N))$ with respect to its arguments $u(i), i=1, \ldots, N$. From $(2.61)$ this function is seen to be symmetric at all the variable vectors. We denote by $u^{*}(i)$ the minimizing vector $u(i)$ such that the minimum is attained as

$$
J^{*}(u)=J\left(u^{*}(1), \ldots, u^{*}(N)\right)
$$

One way to minimize the cost function $J(u)$ is by first determining $u^{*}(1)$, then $u^{*}(2)$ etc., up to $u^{*}(N)$. Suppose that $u^{*}(1)$ was determined 
then

$$
\begin{aligned}
J^{\prime}(u) & =\min _{u(1)} J(u) \\
& =\operatorname{tr}\left[X^{-1}(1)+C^{\top}\left(u^{*}(1)\right) R^{-1}(1) C\left(u^{*}(1)\right)+\sum_{i=2}^{N} C^{T}(u(i)) R^{-1}(i) C(u(i))\right]^{-1} \\
& =\operatorname{tr}\left[X^{*-1}(2)+\sum_{i=2}^{N} C^{T}(u(i)) R^{-1}(i) C(u(i))\right]^{-1}
\end{aligned}
$$

Now

$$
J^{\prime}(u) \leq J(u) \quad \forall u(i), i=2, \ldots, N
$$

and in particular set $u(i)=0, i=2, \ldots, N$. Then

$$
J^{\prime}(u)=\min _{u(1)} \operatorname{tr} X(2), \text { given } X(1)
$$

Next determine $u^{*}(2)$. Then

$$
\begin{aligned}
J^{2}(u)= & \min _{v(2)} J^{1}(u) \\
= & \operatorname{tr}\left[X^{*-1}(2)+C^{\top}\left(u^{*}(2)\right) R^{-1}(2) C\left(u^{*}(2)\right)\right. \\
& \left.+\sum_{i=3}^{N} C^{\top}(u(i)) R^{-1}(i) C(u(i))\right]^{-1} \\
= & \operatorname{tr}\left[X^{*-1}(3)+\sum_{i=3}^{N} C^{\top}(u(i)) R^{-1}(i) C(u(i))\right]^{-1}
\end{aligned}
$$


$J^{2}(u) \leq J^{1}(u) \leq J(u) \quad \forall u(i), \quad i=3, \ldots, N$

and set in particular $u(i) 0, i=3, \ldots, N$. Then

$$
J^{2}(u)=\min _{u(2)} \operatorname{tr} X(3), \text { given } X *(2)
$$

and carrying this procedure to the end, we arrive at

$$
\begin{aligned}
J^{N}(u)=J^{*}(u)= & \min _{u(1), \ldots, u(N)} J(u(1), \ldots, u(N))
\end{aligned}
$$

the required optimum, which is achieved by the sequential one-stage minimization:

$$
\min _{u(k)} \operatorname{tr} x(k+1) \text {, given } X(k), k=1,2, \ldots, N .
$$

In addition to the computational simplicity of the single-stage optimization method, it has another significant advantage. While the dynamic procedure solves for an input sequence of length $N$, where $N$ is a fixed integer, the stage optimization method con be applied on-line, and can be terminated when the error covariance trace function decreases under a certain limit. For the case where $Q=0$, see Appendix B.

\subsection{Properties of the Estimator}

Some properties of the identified model and filter, under the optimal input policy are of interest. For easy reference we rewrire the model $(2.7),(2.9)$ 


$$
\begin{aligned}
& x(k+1)=x(k)+\omega(k) ; \omega(k) \sim N[0, Q(k)] \\
& y(k)=C(u(k)) x(k)+v(k) ; v(k) \sim N[0, R(k)]
\end{aligned}
$$

For the case where we assume $\omega(k)=0$, which is a practical assumption, especially in the cases where the system is constant, or near constant and the observation sequence is short, we use identity (2.56) repeatedly, as was done in the proof of Theorem 2.1, to obtain

$$
X(k+1)=\left[X^{-1}(1)+\sum_{i=1}^{k} C^{T}(u(i)) R^{-1}(i) C(u(i))\right]^{-1}
$$

\section{Definition $2.1[2]$}

The information matrix of the system (2.65), (2.66) is

$$
\mathcal{L}(k, 1)=\sum_{i=1}^{k} C^{\top}(u(i)) R^{-1}(i) C(u(i))
$$

Using the information matrix Equation (2.67) is rewritten

$$
X(k+1)=\left[X^{-1}(1)+\mathcal{L}(k, 1)\right]^{-1}
$$

In order to be able to estimate all the state variables, the system has to be observable. We give now two definitions of observability which are due to Kalman [ 33$],[34]$. For detailed discussion of the problem of observability of discrete linear systems, and the filtering problem, the reader is referred to [2], [13]. 


\section{Definition 2.2}

The system $(2.65),(2.66)$ is said to be k-completely observable

if, and only if,

$$
£(k, 1)>0 \text { for some } k \geq 1 \text {. }
$$

It is $S$-uniformly completely observable if there exists a positive interger $S$ eand positive constants $\alpha, \beta$ such that

$$
0<\alpha I \leq \mathcal{L}(k, k-S) \leq \beta I
$$

for all $k>S$.

If $A$ and $B$ are matrices then the relation $A>B$ or $A-B>0$, means that $A-B$ is positive definite. We introduce now some notations.

$$
\begin{aligned}
& \text { Let } J_{k} \text { be an indexing set } \\
& J_{k}=\{1,2, \ldots, k\}
\end{aligned}
$$

and let $M_{i_{\lambda}}$ be the composite matrix

$$
M_{i_{\lambda}}=\left[\begin{array}{c}
c\left(i_{1}\right) \\
\vdots \\
c\left(i_{\lambda}\right)
\end{array}\right] ; i_{1}, i_{2}, \ldots, i_{\lambda} \in J_{k}
$$

where $C(i)=C(u(i))$.

In order to be able to estimate all the system parameters we must 
establish the observability property of the system $(2.65),(2.66)$, which is done in the following theorem. It is assumed that the dimension of the observation vector $y$ is smaller than that of the state vector $x$

Theorem 2.2

The system (2.65), (2.66) is k-completely observable, if and only if, the input sequence $\{u(1), \ldots, u(k)\}$ contains vectors $u\left(i_{1}\right), u\left(i_{2}\right)$, $\ldots, u\left(i_{\lambda}\right)$ such that $M_{i_{\lambda}}$ is of maximum rank, $n$ - the demension of $x$.

Proof:

By definition 2.2 the system is k-comple ely observable if, $\mathcal{L}(k, 1)>0$, where $\mathcal{L}(k, 1)=\sum^{k} C^{\top}(i) R^{-1}(i) C(i)$. By definition, $\mathcal{L}(k, 1)$ is positive definite if $i=1$

$\mu=z^{T}\left[\sum_{i=1}^{k} C^{T}(i) R^{-1}(i) C(i)\right] z>0 ; \forall z \neq 0$

where $z$ is a vector of appropriate dimension $n$. Define the vectors

$$
e(i)=C(i) z ; i=1, \ldots, k
$$

and the scalar $\mu$ in (2.73) can be written as

$$
\mu=\sum_{i=1}^{k} e^{T}(i) R^{-1}(i) e(i)
$$

By assumption $R(i)>0$ thus from (2.75), $\mu \geq 0$. 
To prove sufficiency assume that there exists $M_{i_{\lambda}}$ of maximum

rank. Then

$$
M_{i_{\lambda}} z=\left[\begin{array}{c}
c\left(i_{1}\right) \\
\vdots \\
c\left(i_{\lambda}\right)
\end{array}\right] z=\left[\begin{array}{c}
e\left(i_{1}\right) \\
\vdots \\
e\left(i_{\lambda}\right)
\end{array}\right] \neq 0 ; \forall z \neq 0
$$

Hence for every non-zero vector $z$ there exist a non-zero vector $e(i)$, and $\mu>0$. Consequently $\delta(k, 1)>0$ and the system is $k$-completely observable.

To prove necessity, assume that the system is k-completely observable. Then by Equation (2.73) $\quad \dot{\mu}>0 \forall z \neq 0$, and by Equation (2.75) there must exist at least one $e(i) \neq 0, i \in J_{k^{\prime}}$, for every $z \neq 0$. Use. Equation (2.74) to obtain the composite equation

$$
\left[\begin{array}{c}
e\left(i_{1}\right) \\
\vdots \\
e\left(i_{\lambda}\right)
\end{array}\right]=\left[\begin{array}{c}
c\left(i_{1}\right) \\
\vdots \\
c\left(i_{\lambda}\right)
\end{array}\right] z=M_{i_{\lambda}} z \neq 0 ; \forall z \neq 0
$$

which means that there exists an $M_{i}$ of full rank.

Q.E.D.

\section{Corollary 2.1}

The system $(2.65),(2.66)$ is S-uniformly completely observable if and only if, for each subsequence of length $S$ of the input sequence, the necessary and sufficient condition of the Theorem 2.2 is satisfied, and the control vector is bounded 
Proof:

If the necessary and sufficient condition of the theorem is satisfied for each control subsequence of length $S$, then

$$
\mathcal{L}(i, i-S)>0 ; \quad \forall i>S ; \quad i \in J_{k}
$$

and taking $k$ to be finite, there exists $\alpha$ and $\beta$ such that (since $u(i)$ is bounded $\forall$ i)

$$
\mathcal{L}(i, i-S)-\alpha I \geq 0 \text { and } \beta I-\mathcal{L}(i, i-S) \geq 0
$$

for every $i>S, i \in J_{k}$. Thus $(2.70)$ is satisfied.

Corollary 2.2

For the system $(2.65),(2.66)$ to be $k$-completely observable, it is necessary and sufficient that the input sequence of length $k$, has at least $\mathrm{n} / \mathrm{m}$ unequal vectors.

Proof:

Since the rows of the observation matrix $C(i)=\dot{C}(u(i))$ are Polynomials of the elements of the input vector $u(i)$, then the rows of $C(u(i))$ will be linearly independent of the rows of $C(u(i))$ whenever $u(i)=u(i)$. Thus $M_{i}$ is of full rank and by Theorem 22 the system is $k$-completely observable. The following theorem establishes a property of the functional used in the input optimization problem. 
The error functional that was used in the input optimization problem is the trace function of the error covariance matrix, that is

$$
J(u)=\operatorname{tr} X(k+1)
$$

Obviously it contains the sum of the individual error variances of all the parameters estimates. We can show that for the noise free parameters model it is a non-increasing function of time.

Theorem 2.3

For the constant parameter model

$$
\begin{aligned}
& x(k+1)=x(k) \\
& y(k)=C(k) x(k)+v(k)
\end{aligned}
$$

the functional $\operatorname{tr} X(k)$ is a monotone non-increasing function of the stage $k$

Proof:

For the given system assuming $X(1)>0$, the error covariance matrix is given by $(2.67)$ and there readily follows that

$$
X(k)>0, \forall k \text {. }
$$

Thus also

$$
C(k) X(k) c^{\top}(k) \geq 0 \text {. }
$$


and assuming $R(k)>0$ also

$$
C(k) X(k) C^{\top}(k)+R(k)>0
$$

Using now Equation (2.29) for the covariance matrix we have

$$
\begin{aligned}
X(k+1)= & x(k) C^{\top}(k)\left[C(k) x(k) C^{\top}(k)\right. \\
& +R(k)]^{-1} C(k) x(k)
\end{aligned}
$$

Denoting

$$
\begin{aligned}
& Y(k)=X(k) C^{\top}(k) \\
& B(k)=C(k) X(k) C^{\top}(k)+R(k)
\end{aligned}
$$

Equation (2.73) becomes

$$
X(k+1)=X(k)-Y(k) B(k) Y^{\top}(k)
$$

where $Y(k) B(k) Y^{\top}(k) \geq 0$ since $B(k)>0$. Thus from (2.81) it follows

$$
\operatorname{tr} X(k+1)=\operatorname{tr} X(k)-\operatorname{tr} Y(k) B(k) Y^{\top}(k)
$$

and

$$
\operatorname{tr} X(k+1) \leq \operatorname{tr} X(k)
$$


since $\quad \operatorname{tr} Y(k) B(k) Y^{\top}(k) \geq 0$

Q.E.D.

By the last theorem, the error variance at any stage is not greater than the one in the previous stage, for any input used. The input optimization achieves the fastest decrease of the error covariance.

The next theorem establishes a bound on the error covariance matrix.

Theorem 2.4

If the system (2.69), (2.70) is S-uniformly completely observable, and $X(1)$ is positive definite, then $X(k)$ is uniformly bounded from above by $\Sigma^{-1}(k, 1)$ for all $k>S$ and $X(k)$ vanishes asymptotically, $X(k) \rightarrow 0$ as $k \rightarrow \infty$. Proof:

By Equation (2.68) we have

$$
x^{-1}(k+1)=x^{-1}(k)+\&(k, 1)
$$

and thus

$$
x^{-1}(k+1) \approx \mathcal{L}(k, 1)
$$

Since by assumption the system is S-uniformly completely observable, then for $k>S, \mathcal{S}(k, 1)>0$ and

$$
X(k+1)<s^{-1}(k, 1)
$$

In addition, for large $k$ 


$$
\mathcal{L}(k, 1)=£(s+1,1)+\mathcal{L}(2 s+2, s+2)+\ldots
$$

a sum of positive definite matrices. Thus

$$
X(k+1)<\mathcal{L}^{-1}(k, 1) \rightarrow 0 \text { as } k \rightarrow \infty .
$$

Q.E.D.

Corollary 2.3

Under the conditions of theorem $2.4, X(k)$ does not depend on the initial condition of the covariance matrix $X(1)$, for $k$ sufficientiy large.

Proof:

From Equations (2.84) and (2.87) it follows that

$$
x^{-1}(k+1) \cong \Sigma(k, 1)
$$

for sufficiently large $k$, since $\mathcal{L}(k, 1)$ is a growing sequence of positive definite matrices, and $X(1)$ is constant.

\subsection{The Cramér-Rao Lower Bound}

The Cramér-Rao Lower Bound (CRLB) plays an important role in the area of statistical parameter estimation. We present now a short discussion on its connection with our identification problem. References [35] and [38 ]- [40] are some among many which derive and discuss the ideas and results that we use.

Consider the following problem. We are given the observation 
equation

$$
y(k)=g(k, \xi)+v(k) ; k=1,2, \ldots, N
$$

where $y(k)$ is the observation m-vector, $g(k, \xi)$ is an m-vector function of the parameter n-vector $\xi . \xi$ is a fixed but unknown vector. $v(k)$ is a zero mean stationary, white Gaussian random vector sequence, with the covariance matrix

$$
R=\operatorname{diag}\left[r_{1} r_{2} \ldots r_{m}\right] "
$$

Let

$$
F(y(1), y(2), \ldots, y(N), \xi)=F(y, \xi)
$$

be the cumulative distribution function of the observations. Let $\hat{\xi}$ be any unbiased estimate of $\xi$, made from $y(k), k=1, \ldots, N$. Let $\Gamma(N)$ be the covariance matrix of the estimation error, i.e.,

$$
\Gamma(N)=E\left[(\hat{\xi}-\xi)(\hat{\xi}-\xi)^{\top}\right]
$$

\section{Define}

$$
B=\int\left[\frac{\partial \log F(y, \xi)}{\partial \xi}\right]\left[\frac{\partial \log F(y, \xi)}{\partial \xi}\right]^{\top} d F(y, \xi)
$$

Then the Cramér-Rao or information inequality states, [35 ]- [40 ],

$$
\Gamma(N) \geq B^{-1}
$$

$B$ is the Fisher information matrix, and $B^{-1}$ is the Cramer-Rao lower bound on the 
error covariance matrix. In (2.93), the equality holds for efficient estimators. It can be shown that maximum likelihood estimators are asymptotically efficient, and when the observation is linear in the unknown parameter the estimator (which is the Kalman filter in our case) is efficient so that $\Gamma(N)=B^{-1}$.

Equation (2.93) holds for any $v(k)$ which is some discrete stochastic process, but $B$ is extremely hard to evaluate, since $F(y, \xi)$ is generally not known. But for $v(k)$ which is zero mean, stationary, white discrete Gaussian process with covariance $R$ as in (2.89) B becomes

$$
B=\sum_{k=1}^{N}\left[\frac{\partial g(k, \xi)}{\partial \xi}\right] R^{-1}\left[\frac{\partial g(k, \xi)}{\partial \xi}\right]^{T}
$$

where we note that $\frac{\partial g(k, \xi)}{\partial \xi}$ is an $(n \times m)$ matrix with columns

$$
\frac{\partial g_{i}(k, \xi)}{\partial \xi}, i=1,2, \ldots, m .
$$

Using our observation model (2.9) we have

$$
g(k, \xi)=C(k) \xi
$$

where $\xi=x(k), k=1, \ldots, N$ and $\hat{\xi}=\hat{x}(N+1)$. Hence

$$
\frac{\partial g(k, \xi)}{\partial \xi}=C^{\top}(k)
$$

so that Equations (2.94) and (2.93) become

$$
B=\sum_{k=1}^{N} C^{T}(k) R^{-1} C(k)
$$




$$
\Gamma(N)=\left[\sum_{k=1}^{N} C^{T}(k) R^{-1} C(k)\right]^{-1}
$$

Nahi and Wallis [ 36$]$ determine optimal input for identification of dynamic systems by minimizing a functional of the CRLB. In our case, this would mean for the trace function:

$\min _{u(k) \in \Omega} \operatorname{tr}\left[\sum_{k=1}^{N} C^{\top}(u(k)) R^{-1} C(u(k))\right]^{-1}, k=1, \ldots, N$

In section 2.4.1 we formulated the optimal input problem as

$$
\min _{u(k) \in \Omega} \operatorname{tr} X(N+1) ; k=1, \ldots, N
$$

which for the noise free parameter model

$$
x(k+1)=x(k)
$$

can be written by using (2.67) as

$$
\min _{u(k) \in \Omega} \operatorname{tr}\left[X^{-1}(1)+\sum_{k=1}^{N} C^{\top}(u(k)) R^{-1} C(u(k))\right]^{-1} ; k=1, \ldots, N
$$

and since $X^{-1}(1)$ is constant the optimal input sequence that satisfies (2.99), also satisfied (2.101) and thus minimizes the trace of the CRLB. This is the best any input sequence can do, under the trace functional, irrespective of the filter that is used to estimate the parameters. 


\subsection{System Simulation}

Several systems were simulated, and the optimal input sequence obtained by straightforward application of the stage minimization method. The following is one example.

\subsubsection{Example}

The optimal input sequence is calculated in this example for a system with two inputs and two outputs. The inputs-output relation is a second order polynomial

$$
\begin{aligned}
& y_{2}(k)=a_{11} u_{1}^{2}(k)+a_{12} u_{2}^{2}(k)+a_{13} u_{1}(k)+a_{14} u_{2}(k)+v_{1}(k) \\
& y_{2}(k)=a_{21} u_{1}^{2}(k)+a_{22} u_{2}^{2}(k)+a_{23} u_{1}(k)+a_{24} u_{2}(k)+v_{2}(k)
\end{aligned}
$$

where the parameters $a_{i j}$ are unknown constants. The measurement vector $y(k)$ is generated by this system with

$$
\begin{aligned}
& v(k)=\left[\begin{array}{ll}
v_{1}(k) & v_{2}(k)
\end{array}\right]^{\top} \\
& v(k) \sim N[0, R] ; R=\operatorname{diag}\left[\begin{array}{ll}
2.0 & 1.0
\end{array}\right]
\end{aligned}
$$

The parameters are modeled as

$$
x(k+1)=x(k)
$$

where

$$
x(k)=\left[a_{11}(k) \ldots a_{14}(k) a_{21}(k) \ldots a_{24}(k)\right]^{\top}
$$


and the observation equation is

$$
y(k)=C(k) \times(k)+v(k)
$$

where

$$
\begin{aligned}
& y(k)=\left[\begin{array}{ll}
y_{1}(k) & y_{2}(k)
\end{array}\right]^{\top} ; u(k)=\left[\begin{array}{ll}
u_{1}(k) & u_{2}(k)
\end{array}\right]^{\top} \\
& C(k)=\left[\begin{array}{cccccccc}
u_{1}^{2}(k) & u_{2}^{2}(k) & u_{1}(k) & u_{2}(k) & 0 & 0 & 0 & 0 \\
0 & 0 & 0 & 0 & u_{1}^{2}(k) & u_{2}^{2}(k) & u_{1}(k) & u_{2}(k)
\end{array}\right]
\end{aligned}
$$

The initial covariance matrix is taken to be large,

$$
X(1)=\left[\begin{array}{cccc}
100 & 20 & \ldots & 20 \\
20 & 100 & \ldots & 20 \\
\vdots & & & \vdots \\
20 & 20 & \ldots & 100
\end{array}\right]
$$

and the control has the saturation constraint

$$
\left|u_{i}(k)\right| \leq \text { SAT } i=1,2, k=1,2, \ldots
$$

The optimal input sequence of length $8,\{u(1), \ldots, u(8)\}$, is computed by the stage minimization method, for two control saturation constraints
(1) SAT $=1$.
(2) $\quad$ SAT $=5$. 
The solution does not depend on the actual system observations, but only on the assumed model and noise statistics. It is not unique, as can be expected from the local convergence properties of all gradient numerical techniques. Starting at each stage-minimization with an initial guess of 0.5 for the two control components, we arrive at the solution shown in Figure 2.1 for SAT $=1$, and Figure 2.2 for SAT = 5. The initial value of the cost function is $\operatorname{tr} X(1)=800$, and it is reduced to 4.559 in the first case and to 0.0815 in the second case.

The two optimal input sequences are applied to two systems having two different sets of parameters, and the estimates as well as the absolute percentage errors are averaged over 50 runs with randomly selected noise samples. These averages are compared for the cases of SAT $=1$, and SAT $=5$ in Table 2.1. As can be expected the control with the larger saturation constraint yields smaller identification errors, and better average estimates. The estimates are those obtained by filtering eight system observations, $\{y(1), \ldots, y(8)\}$, and thus are the elements of the vector $\hat{x}(9 \mid 8)$. The initial estimates are taken as

$$
\hat{x}(1)=[4.0 \ldots 4.0]^{\top}
$$

and the error in the parameter estimates is calculated as

$$
\epsilon_{1}=\left|\frac{\hat{a}_{i}-a_{i}}{a_{i}}\right| \cdot 100 ; \quad i=1, \ldots, 8
$$

The conjugate gradients method stops the search iterations whenever the improvement in the cost at two consecutive iterations is smaller than a certain 

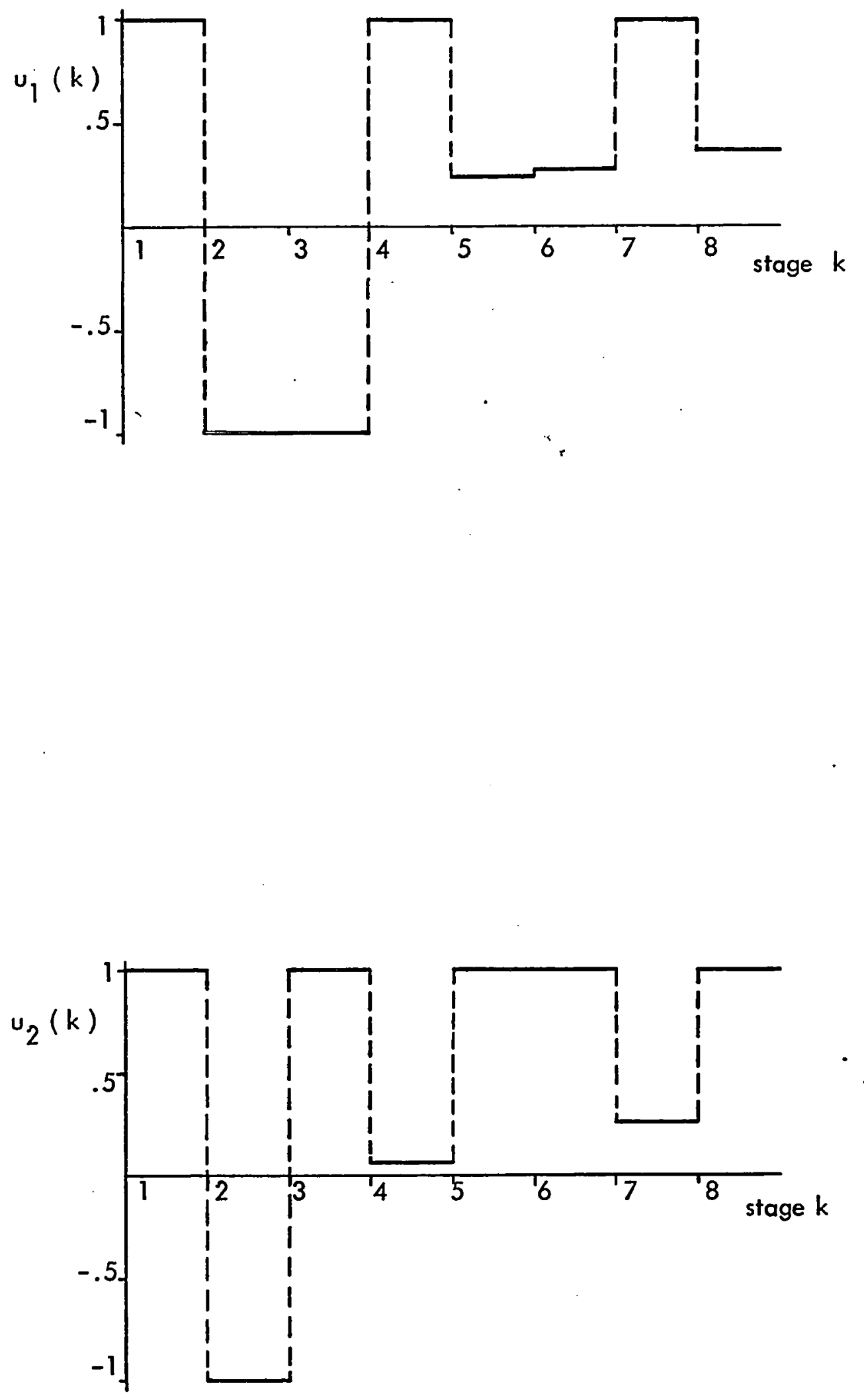

FIGURE 2.1 OPTIMAL INPUT FOR CONTROL-BOUND SAT $=1$ 

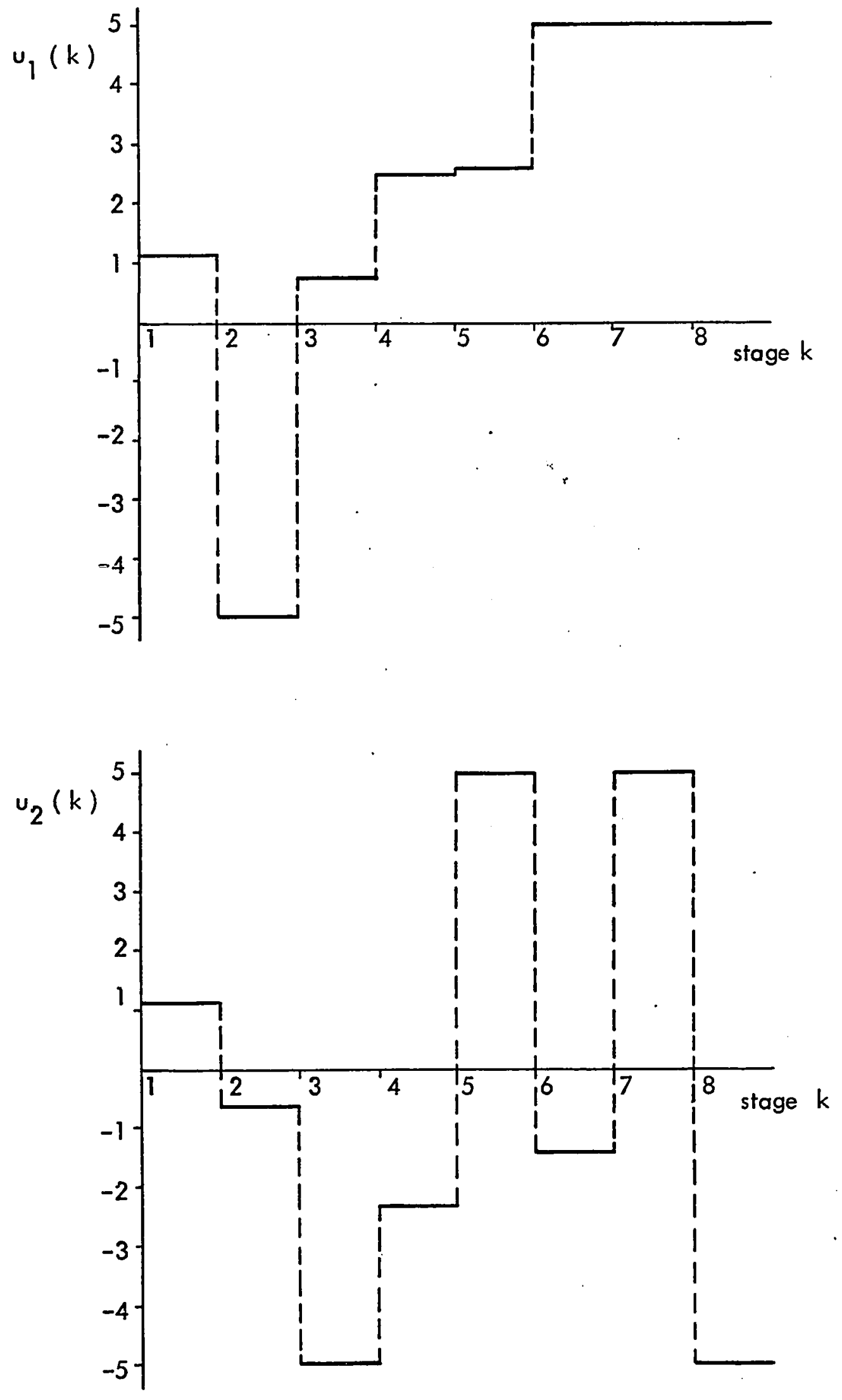

FIGURE 2.2 OPTIMAL INPUT FOR CONTROL-BOUND SAT=5. 


\begin{tabular}{|c|c|c|c|c|}
\hline $\begin{array}{c}\text { actual } \\
\text { parameters }\end{array}$ & $\begin{array}{l}\text { average } \\
\text { estimates }\end{array}$ & $\begin{array}{c}\text { AT }=5 . \\
\text { average } \\
\text { errors }\end{array}$ & $\begin{array}{l}\text { input } \\
\text { average } \\
\text { estimates }\end{array}$ & $\begin{array}{l}\text { SAT }=1 . \\
\begin{array}{c}\text { average } \\
\text { errors }\end{array}\end{array}$ \\
\hline$a_{11}=3.0$ & 3.01 & 1.02 & 2.85 & 24.81 \\
\hline$a_{12}=-4.0$ & -4.00 & 0.77 & -3.73 & 28.12 \\
\hline$a_{13}=6.0$ & 6.02 & 2.46 & 6.28 & 12.11 \\
\hline$a_{14}=-1.5$ & -1.47 & 5.46 & -1.75 & 54.26 \\
\hline$a_{21}=-0.5$ & -0.49 & 4.45 & -0.61 & 107.8: \\
\hline$a_{22}=1.5$ & 1.49 & 1.66 & 1.64 & 45.80 \\
\hline$a_{23}=0.8$ & 0.82 & 13.22 & 0.94 & 55.39 \\
\hline$a_{24}=2.5$ & 2.52 & 3.39 & 2.33 & 24.58 \\
\hline
\end{tabular}

\section{System 1}

\begin{tabular}{|rr|r|r|r|r|}
$a_{11}=$ & 0.3 & 0.31 & 10.25 & 0.20 & 248.13 \\
$a_{12}=-1.5$ & -1.50 & 2.06 & -1.30 & 75.25 \\
$a_{13}=3.0$ & 3.02 & 4.92 & 3.26 & 24.09 \\
$a_{14}=-4.0$ & -3.97 & 2.04 & -4.20 & 19.97 \\
$a_{21}=12.0$ & 12.01 & 0.19 & 11.84 & 4.59 \\
$a_{22}=4.0$ & 3.99 & 0.62 & 4.18 & 17.23 \\
$a_{23}=-0.5$ & -0.48 & 21.16 & -0.34 & 90.37 \\
$a_{24}=1.5$ & 1.52 & 5.65 & 1.31 & 41.18 \\
\hline
\end{tabular}

System 2

TABLE 2.1 PARAMETER ESTIMATES AND ERRORS. 
value, where the last search is in the direction of steepest descent. After several conjugate gradient steps the solution algorithm switches to ordinary gradient search for one or more iterations before continuing with conjugate gradients. This action is found to improve the rate of convergence toward the final solution.

The simulation was done using the IBM $360 / 75$. The execution time was 11.29 seconds for this example. 


\section{CHAPTER III}

\section{DUAL CONTROL OF DYNAMIC}

\section{LINEAR STOCHASTIC SYSTEMS}

\subsection{Introduction}

Using the approach of $\AA$ ström, Wittenmark and Wieslander $[3,5]$, an adaptive control law is derived for the regulation problem of a linear stochastic system. The system is modeled by a dynamic discrete input-output relation with one input and one output, with parameters which are assumed to be stochastic processes. For systems with known parameters the output regulator consists of the minimum-variance policy $[1]$. A natural extension to the case of unknown parameters is still the minimum-variance policy, with the estimates substituted for the unknown parameters. This policy however, completely separates the identification and control, and the control is computed with complete disregard to the identification error variance.

The adaptive regulator policy takes into consideration the error variance of the parameter estimates and adapt itself, in producing the control signals, to the uncertainty of the estimation. It is compared by Wittenmark [ 5$]$, with the extended minimum variance regulator that uses the parameter estimates only, and is found superior.

The adaptive control policy is derived by minimizing the expected value of the squared output at each stage. The result is a feedback law, which depends 
on the system parameter estimates and their error variance. But the estimation and control are separated and the controller does not act in any way to improve the identification. Thus it is not dual $[16,17]$, and allows two kinds of phenomena to occur, which result in low performance. These are burst and turnoff phenomena. Wittenmark and Wieslander $[4,5]$ use the adaptive control with additive perturbation signal to improve the parameter estimation and hence improve the controller performance.

Our motivation is to design the control signal by considering directly the estimation error covariance, as well as the expected squared output. We use a measure of this error covariance to constrain the control signal in such a way that it acts to keep good estimation together with good control.

Since our controller, and the one used by Wittenmark and Wieslander, are concerned with both identification and control they are of dual nature (Feldbaum [16]). But the perturbation added to the adaptive control signal contributes to improving the identification in a passive way, while our controller seeks and plays an active role in supervising the identification - process.

We start by developing the adaptive non-dual control law, and consequently, formulate and design the adaptive dual control policy. 


\subsection{Formulation of the Problem}

A discrete linear mociel is considered with scalar input and scalar output. The model describes the input-output relation in the plant

$$
\begin{aligned}
y(k)= & a_{1}(k) y(k-1)+a_{2}(k) y(k-2)+\ldots+a_{n}(k) y(k-n) \\
& +b_{1}(k) \cup(k-1)+b_{2}(k) u(k-2)+\ldots b_{n}(k) \cup(k-n) \\
& +v(k)
\end{aligned}
$$

where

$k=1,2, \ldots$ discrete time or stage index

$y(k)$ - output

$u(k)$ - input (control)

$a_{i}(k), b_{i}(k), i=1, \ldots, n$ time varying unknown parameters

$v(k), k=1,2, \ldots$ a sequence of equally distributed, independent,

normal random variables, with zero mean and variance $R$,

$$
\begin{aligned}
& v(k) \sim N(0, R), k=1,2, \ldots \\
& \operatorname{cov}[v(i), v(i)]=R U_{0}(i-i), i, i=1,2, \ldots
\end{aligned}
$$

where

$$
U_{0}(i-i) \text { is the discrete impulse function }
$$$$
U_{0}(i-i)= \begin{cases}1 & i=i \\ 0 & i \neq i\end{cases}
$$

We define as admissible, any control law, such that the control signal at stage $k$ is a function of the observed outputs to stage $k$, and past control signals 
to time $k-1, i . e, u(k)$ is a function of $y(k), y(k-1), \ldots, u(k-1)$, $u(k-2), \ldots$

We wish to design an optimal controller, that will keep the output of the system as near zero as possible. This is the regulator problem. A suitable performance index to be minimized is

$$
V_{N}=E\left[\sum_{k=1}^{N} y^{2}(k)\right]_{r}
$$

The minimization of this loss function yields an $\mathrm{N}$-stage control policy which is of dual nature $[16],[17]$. It not only computes the best control, based on past and present observations, but also acts to improve the system identification and increase the information about the system.

This problem, however, leads to a dynamic programming solution which does not possess an analytic form, and which can not be obtained in real time even for a first order system, $[3],[5]$.

To arrive at an on-line solution we choose the single-stage performance index

$$
V=E\left[y^{2}(k)\right]
$$

It turns out that the solution using this loss function separates the system indentification and control parts. The control law will make the predicted value of $y(t)$ equal to zero, but will not make any attempt to improve the 
parameter estimates. Thus it is not a dual control. The result of this may be, as will be later demonstrated, an unsatisfactory controller. By adding a certain requirement involving the identification quality, we later develop a technique for a dual control strategy.

The control law that minimizes the loss function $(3.3)$ is called optimal non dual control.

\subsection{The Optimal Non-Dual Control Law}

Defining the vector

$$
x(k)=\left[a_{1}(k) \ldots a_{n}(k) b_{1}(k) \ldots b_{n}(k)\right]
$$

We rewrite the modei equation (3.i)

$$
y(k)=\varphi(k-1) \times(k)+v(k)
$$

where,

$$
\varphi(k-1)=[y(k-1) \ldots y(k-n) \cup(k-1) \ldots v(k-n)]
$$

The unknown parameiers are assumed to be stochastic, Gauss-Markov

processes

$$
x(k+1)=x(k)+\omega(k)
$$

where $\omega(k)$ is a sequence of independent, equally distributed, normal vector random variables, with zero mean and covariance matrix $Q$,

$$
\omega(k) \sim N[0, Q]
$$


The adaptive control problem:

$\min E\left[y^{2}(k+1)\right]$

$u(k)$

subject to equations (3.5) and (3.7).

To proceed we make use of a fundamental lemma of stochastic control theory. Following Aström [1] let $x \in X$ and $y \in Y$ be a vector and $a$ scalar random variables respectively, defined on a probability space, and let the control variable be $u \in U$. Let the loss function $g$ be a function which maps $X \times Y X U$ into the real numbers. The expected loss is $E[g(x, y, u)]$ where $E$ is the mathematical expectation with respect to $x$ and $y$. We are interested in the minimization of the expected loss $E[g(x, y, u)]^{\prime}$, with respect to the control variable $v \in U$, which is restricted to all functions that map $Y$ into $U$. Let $\min E[g(x, y, u)]$ denote the minimum of $E[g(x, y, u)]$ with respect to $u(y)$ all admissable control strategies.

Lemma 3.1 (Aström)

Let $E[\cdot \mid y]$ denote the conditional mean given. y. Assume that the function $f(y, u)=E[g(x, y, u) \mid y]$ has a unique minimum with respect to $u \in U$ for all $y \in Y$. Let $u^{\circ}(y)$ denote the value of $u$ for which the minimum is achieved. Then

$$
\begin{aligned}
\min _{u(y)} E[g(x, y, u)] & =E\left[g\left(x, y, u^{\circ}(y)\right)\right] \\
& =E_{y}\{\min E[g(x, y, u) \mid y]\}
\end{aligned}
$$

where $E_{y}$ denote the mean value with respect to the distribution of $y$. 
Since $E[\cdot \mid y]$ is a function of $y$, and the operation $\min f(x, u)$ defines a function $x \rightarrow U$, we note that the operation $\min E[\cdot \mid y]$ $u$ thus defines a function $Y \rightarrow U$.

Using lemma 3.1, expression (3.8) is equivalent to

$$
\min _{u(k)} E\left[y^{2}(k+1) \mid y_{k}\right]
$$

subject to equations (3.5) and (3.7), where

$$
y_{k}=\{y(k) y(k-1) \ldots u(k-1) u(k-2) \ldots\}
$$

is the collection of past measurements and control values.

The expected loss of (3.8) is a function of the collection $y_{k}$ as well as the systems parameters. We know only $y_{k}$ and thus use the fundamental lemma 3.1 to obtain the admissible minimizing control $u(k)=u(y(k), y(k-1)$, $\ldots, u(k-1), u(k-2), \ldots)$.

The solution of (3.9) proceeds as follows:

$$
\begin{aligned}
V^{0}= & \min _{u(k)} E\left[y^{2}(k+1) \mid y_{k}\right] \\
= & \min _{u(k)} E\left[\left(a_{1}(k+1) y(k)+\ldots+a_{n}(k+1) y(k-n+1)\right.\right. \\
& \left.+b_{1}(k+1) u(k)+\ldots+b_{n}(k+1) u(k-n+1)+v(k+1)^{2} \mid y_{k}\right] \\
= & \min E\left[b_{1}^{2}(k+1) u^{2}(k)+2 b_{1}(k+1) u(k) a^{\top}(k+1)\right. \\
& \left.\cdot Y(k)+2 b_{1}(k+1) u(k) v(k+1) \mid y_{k}\right] \\
& + \text { terms independent of } u(k)
\end{aligned}
$$


where we define the vectors

$$
\begin{aligned}
& a_{c}(k)=\left[a_{1}(k) a_{2}(k) \ldots a_{n}(k) 0 b_{2}(k) b_{3}(k) \ldots b_{n}(k)\right]^{\top} \\
& Y(k)=[y(k) y(k-1) \ldots y(k-n+1) 0 u(k-1) u(k-2) \ldots u(k-n+1)]^{\top}(3.13)
\end{aligned}
$$

We define now

$$
\begin{aligned}
\hat{x}(k \mid i) & =E\left[x(k) \mid y_{i}\right] \\
\tilde{x}(k \mid i) & =x(k)-\hat{x}(k \mid i) \\
P(k \mid i) & =\operatorname{cov}[\tilde{x}(k \mid i), \tilde{x}(k \mid i)] \\
& =E\left[\tilde{x}(k \mid i) \tilde{x}^{\top}(k \mid i)\right]
\end{aligned}
$$

which are recognized as the optimal Kalman Filter state estimate, error and error covariance matrix, for the linear system of equations (3.7) and (3.5).

Using (3.15) equation (3.11) becomes

$$
\begin{aligned}
V^{0}=\min _{u(k)} E & {\left[\left(\hat{b}_{1}(k+1 \mid k)+\tilde{b}_{1}(k+1 \mid k)\right)^{2} u^{2}(k)+2\left(\hat{b}_{1}(k+1 \mid k)\right.\right.} \\
+ & \left.\tilde{b}_{1}(k+1 \mid k)\right) u(k) v(k+1)+2\left(\hat{b}_{1}(k+1 \mid k)\right. \\
& \left.+\tilde{b}_{1}(k+1 \mid k)\right) u(k)\left(\hat{a}_{c}^{\top}(k+1 \mid k)+\tilde{a}_{c}^{\top}(k+1 \mid k)\right) \\
& \left.\cdot Y(k) \mid y_{k}\right] \\
& + \text { terms indepdent of } u(k) .
\end{aligned}
$$

Using (3.14) and (3.16) and taking the conditional expectation

$$
v^{0}=\min _{u(k)}\left[\left(\hat{b}_{1}^{2}(k+1 \mid k)+P_{b_{1} b_{1}}(k+1 \mid k)\right) u^{2}(k)\right.
$$




$$
\begin{aligned}
& \left.+2\left(\hat{b}_{1}(k+1 \mid k) \hat{a}_{c}^{\top}(k+1 \mid k)+P_{n+1}^{\top}(k+1 \mid k)\right) Y(k) u(k)\right] \\
& + \text { terms indepdent of } u(k)
\end{aligned}
$$

where

$$
\begin{aligned}
& \hat{a}_{c}(k+1 \mid k)=\left[\hat{a}_{1}(k+1 \mid k) \ldots \hat{a}_{n}(k+1 \mid k) 0 b_{2}(k+1 \mid k) \ldots b_{n}(k+\mid k)\right]^{\top}(3 \\
& P_{b_{1} b_{1}}(k+1 \mid k)=\operatorname{cov}\left[\tilde{b}_{1}(k+1 \mid k), \tilde{b}_{1}(k+1 \mid k)\right]=E\left[\tilde{b}_{1}^{2}(k+1 \mid k)\right]
\end{aligned}
$$

$P_{b_{1} b_{1}}(k+1 \mid k)$ is the $(n+1) \times(n+1)$ element of $P(k+1 \mid k)$, and $P_{n+1}(k+1 \mid k)$ is the $n+1$ column of the matrix $P(k+1 \mid \dot{k})$. Note that the transition from (3.17) to $(3.18)$ used the orthogonal projection property of the optimal filter - the estimate and error are orthogonal, i.e.,

$$
E\left[\hat{x}^{\top}(i \mid i) \tilde{x}(i \mid i)\right]=0
$$

by the orthogonal projection lemma [6]. Equation (3.21) also holds componentwise [2].

The minimizing $u(k)$ is now found by differentiating the expression to be minimized in $(3.18)$ and equating the result to zero.

$$
\begin{aligned}
& 2\left(\hat{b}_{1}^{2}(k+1 \mid k)+P_{b_{1} b_{1}}(k+1 \mid k)\right) u(k)+2\left(\hat{b}_{1}(k+1 \mid k) \hat{a}_{c}^{\top}(k+1 \mid k)\right. \\
& \left.+P_{n+1}^{\top}(k+1 \mid k)\right) Y(k)=0
\end{aligned}
$$

yielding the optimal control $u^{\circ}(k)$

$$
u^{\circ}(k)=-\frac{\left[\hat{b}_{1}(k+1 \mid k) \hat{a}_{c}^{\top}(k+1 \mid k)+P_{n+1}^{\top}(k+1 \mid k)\right]}{\hat{b}_{1}^{2}(k+1 \mid k)+P_{b_{1} b_{1}}(k+1 \mid k)} Y(k)
$$


This is the optimal non dual control law. It is not acting to improve the parameter estimation.

It is adaptive and depends not only on the predicted values of the parameters, but also on the measure of uncertainty in their estimation - the error variances of the parameters prediction.

The estimates and error variance are obtained using the Kalman filter equations (e.g. [2 ]), with the system (3.7) and observation (3.5).

$$
\begin{aligned}
& \hat{x}(k+1 \mid k)=\hat{x}(k \mid k) \quad ; \quad x(1 \mid 0)=\bar{x} \\
& P(k+1 \mid k)=P(k \mid k)+Q ; \quad P(1 \mid 0)=\bar{P} \\
& \hat{x}(k \mid k)=\hat{x}(k \mid k-1)+K(k)[y(k)-\varphi(k-1) \hat{x}(k \mid k-1)] \\
& P(k \mid k)=P(k \mid k-1)-K(k) \varphi(k-1) P(k \mid k-1) \\
& K(k)=P(k \mid k-1) P^{\top}(k-1)\left[\varphi(k-1) P(k \mid k-1) \varphi^{\top}(k-1)+R\right]^{-1}
\end{aligned}
$$

The foregoing is summarized in the following theorem.

Theorem 3.1

For the system given by equations (3.5) and (3.7), let the admissible control $u(k)$ be a function of past iniputs and outputs, up to stage $k$. Then the control law that minimizes the criterion $(3.3)$ is given by (3.22) and the Kalman filter equations $(3.23)-(3.27)$.

The choice of performance criterion (3.3) separates the identification 
and control parts. The parameters are first predicted as $\hat{x}(k+l \mid k)$ and the control is then computed using these predicted values, and their estimation error variances. If however the identification is poor then the control will be computed in error and in addition, nothing will be done by the controller to improve the identification. If the estimation was perfect then the error variances are all equal to zero, and the controller reduces to the minimum variance strategy $[1]$, with the estimates substituted for the true parameters. Here the error variances are added to the estimates and thus influence their relative weight in (3.22), according to their accuracy.

A serious drawback in the use of this non dual adaptive control law is the frequent occurence of the turn off and burst phenomena. These phemomena were noticed and investigated by Aström, Weislander and Wittenmark [3 ] - [5 ].

The burst phenomenon may occur after a period of good identification. The estimates are then close to the plants parameters and the error variances are small. If the plants parameters change in such a way, that the identifier is too slow in catching up, large output values occur which result in large input signals as well. This in turn improves the identification and reduces the output values.

The burst phenomenon is especially sensitive to the gain parameter estimate. From the control law (3.22) it is seen that if the gain is identified as a small number (this can easily happen even if $b_{1}$ is not very small) and if its estimate error variance $P_{b_{1} b_{1}}$ is at the same time small too, then the controller (3.22) produces large values and the output oscillates with large signals. 
The turn-off phenomenon shuts off the controller, in the sense that the control values are almost zero (in computer simulations these values were of the order $\left.10^{-4}-10^{-8}\right)$. The reason for the control turn-off can be explained as follows. If after a period of good estimation the gain parameter $b(k)$, and then also its estimate $\hat{b}(k)$, become small over a period of time, then the control $u^{\circ}(k)$ will be small too and consequently the filter gain $\mathrm{K}_{b_{1}}(k)$ will decrease, as there is not much information about $b_{1}(k)$ in the observation equation. This in turn may result in the next estimate being even smaller while the error variance $P_{b_{1} b_{1}}(k+1 \mid k)$ will increase, and $u(k+1)$ and $K(k+1)$ decrease further. The controller may remain in the turn-off mode, or as frequently occurs, switch between the turn-off and turn-on modes. It was observed during systems simulations that the turn-off occurs when the gain parameter becomes small or crosses zero while switching signs. In these cases $\hat{b}_{1}(k)$ quickly became very small, of the order $10^{-5}-10^{-8}$. The turn-off occurred also in some cases when the gain $b_{1}(k)$ was not small but was poorly identified as a small number. Thus the small $\hat{b}_{j}(k)$ together with large $P_{b_{1} b_{1}}(k+1 \mid k)$ result in small control signal and small filter gain which does not improve and leaves the bad estimate at its low value, while the error variance increases.

The turn-off phenomenon will terminate if, as a result of virtually no control, the output signal will increase to a large enough value such that the control will increase, being a function of the output, and improve the gain parameter identification. 
Wittenmark and Wieslander [4] give an explanation to this phenomenon by observing that the identifier, being a Kalman filter acis to whiten the innovation process $[10]$. For a system with two parameters $a$ and $b$,

$$
\begin{aligned}
\because(k) & =y(k)-\varphi(k-1) \hat{x}(k \mid k-1) \\
& =y(k)-y(k-1) \hat{a}(k \mid k-1)-u(k-1) \hat{b}(k \mid k-1) \\
& =y(k-1)[a(k)-\hat{a}(k \mid k-1)]+u(k-1)[b(k)-\hat{b}(k \mid k-1)]+v(k)
\end{aligned}
$$

This can be done in two ways. Making the estimates $\hat{a}(k \mid k-1)$ and $\hat{b}(k \mid k-1)$ as close to $a(k)$ and $b(k)$ as possible, or making $\hat{b}(k \mid k-1)$ and consequently $u(k-1)$ almost equal to zero, while $y(k-1)$ is small. In either case

$$
v(k) \cong v(k)
$$

a white noise process.

A rigorous analysis of this phenomenon is extremely difficult, since the system with the adaptive controller (3.22), and the filter equations constitute a complex nonlinear stochastic process.

In general we see that there is a conflict here between identification and control. For good identification there is a need for large control signals (large signal to noise ratio) while the minimum output variance strategy, requires small control values. Wieslander and Wittenmark $[4],[5]$, suggest a method to overcome the above-mentioned drawbacks of the adaptive non dual controller. They argue that since the controller is good if the identification part works well, a small signal which is time varying may be added to the control, thus obtaining a suboptimal dual adaptive confroller. 
The additional small signal can be a square wave or a pseudo random binary sequence. This perturbation while adding somewhat to the loss function keeps the system stimulated and improves the identification. They investigate $[4],[5]$ the perturbation controller

$$
-u^{P}(k)=u^{0}(k)+(-1) \cdot \delta
$$

where $\delta$ is some constant arrived at through system simulation and experimentation. This square wave perturbation controller is simulated and compared to the non dual controller. Their results show that the perturbation controller successfully prevents the turn-off and burst phenomena in their examples.

In the next section we develop a new technique for a suboptimal dual adaptive control.

\subsection{A Technique for Dual Adaptive Control}

As is demonstrated by Wittenmark and Wieslander $[4,5]$, the perturbation controller improves the performance mainly by preventing the turnoff phenomenon. The pertubation signal does not allow the control to be completely turned off, and thus maintains continuous tracking of the gain parameter. It is not however, concerned with improving in general the identification, and is not of active dual nature in this sense 
We are thus motivated to seek a controller that will not only control the system output, but one that will also observe the estimation error covariance and will actively maintain it at a low leve!.

Substituting (3.27) in (3.26), obtain

$$
\begin{aligned}
P(k \mid k)= & P(k \mid k-1)-P(k \mid k-1) \varphi(k-1)\left[\varphi(k-1) P(k \mid k-1) P^{\top}(k-1)+R\right]^{-1} \\
& \varphi(k \mid k-1)
\end{aligned}
$$

and after applying lemma 2.1 this becomes $(P(k \mid k-1), R>0)$

$$
P^{-1}(k \mid k)=P^{-1}(k \mid k-1)+\varphi^{\top}(k-1) R^{-1} \varphi(k-1)
$$

The error covariance matrix of the parameter estimate $\hat{x}(k+1 \mid k)$ appearing in the control law (3.22) is $P(k+1 \mid k)$ and is given as a function of $P(k \mid k)$ by equation (3.24).

$$
P(k+1 \mid k)=P(k \mid k)+Q
$$

Our intention is to modify the control law (3.22) in order to improve the identification of the random system parameters, and thus to improve the overall performance of the controller. To achieve that we wish to monitor some functional of the identification error variance, which indicates the quality of the parameter estimates. We then would like the control law to act in relation to the measure of uncerfainty, in such a way that the identification error remains small. This is to be accomplished in real-time, and hence we require this error functional to be such a function of the control signal that will permit us to compute $u(k)$ analytically. 
The matrix $\mathrm{P}^{-1}(k \mid k)$ in $(3.31)$ is related to the information matrix of definition 2.1 [ $[2]$. It is actually identical to $\{(k, 1)$ of definition 2.1 for the case where the parameters are constants, $Q=0 . \quad P^{-1}(k \mid k)$ contains a measure of the information available about the system parameters. It is also a function of the control. It is in addition, the inverse of the error matrix, and hence the following functional

$$
\rho(k+1)=\operatorname{tr}^{-1}(k+1 \mid k+1)
$$

satisfies all the requirements mentioned above as it is an estimation error functional, and at the same time a quadratic function of the control as is seen by using equation (3.31).

$$
\rho(k+1)=\operatorname{tr} P^{-1}(k+1 \mid k+1)=\operatorname{tr} P^{-1}(k+1 \mid k)+\operatorname{tr} \varphi^{\top}(k) R^{-1} \varphi(k)
$$

using the identity

$$
\operatorname{tr} z z^{T}=z^{\top} z
$$

for any vector $z$, equation $(3.34)$ becomes

$$
P(k+1)=\operatorname{tr} P^{-1}(k+1 \mid k)+R^{-1} \varphi(k) \varphi^{\top}(k)
$$

from equations (3.6) and (3.13) we have

$$
\begin{aligned}
\varphi(k) \varphi^{\top}(k)= & y^{2}(k)+\ldots+y^{2}(k-n+1)+u^{2}(k)+u^{2}(k-1) \\
& +\ldots+u(k-n+1) \\
= & u^{2}(k)+Y^{\top}(k) Y(k)
\end{aligned}
$$


thus (3.35) becomes

$$
\rho(k+1)=\operatorname{tr} P^{-1}(k+1 \mid k)+R^{-1}\left[u^{2}(k)+Y^{\top}(k) Y(k)\right]
$$

We are now ready to formulate the new adaptive control problem.

Dual Control problem formulation:

Choose the control $u(k)$ to minimize the loss function

$$
v=E\left[y^{2}(k+1) \mid k\right]
$$

subject to the constraint

$$
\rho(k+1)=\operatorname{tr} P^{-1}(k+1 \mid k+1) \geq \bar{\rho}(k+1)
$$

where $\bar{\rho}(k+1)$ is a predetermined function.

The constraint (3.39) comes to ensure that the amount of information about the system parameters is keptabove a certain level.

Equation (3.37) yields for the constraint (3.39)

$$
\operatorname{tr} P^{-1}(k+1 \mid k)+R^{-1} Y^{\top}(k) Y(k)+R^{-1} u^{2}(k) \geq \bar{\rho}(k+1)
$$

clearly, from this inequality the control that satisfies the constraint is

$$
|u(k)| \geq \bar{u}(k)
$$

where

$$
\bar{v}(k)=\left|\left[R \bar{\rho}(k+1)-R \operatorname{tr} P^{-1}(k+1 \mid k)-Y^{\top}(k) Y(k)\right]^{1 / 2}\right|
$$


assuming that the square root exists as a real number.

To determine the control law that minimizes the loss function (3.38), and satisfied (3.41), we make use of the diagram in figure 3.1. We plot the part of the loss function which is dependent on $u(k)$. Since we use the same loss function here as in the previous section where the non dual controller was developed, we can use expression (3.18). The first term is quadratic in $u(k)$ and the second is linear in $u(k)$. These are plotted in Figure 3.i.

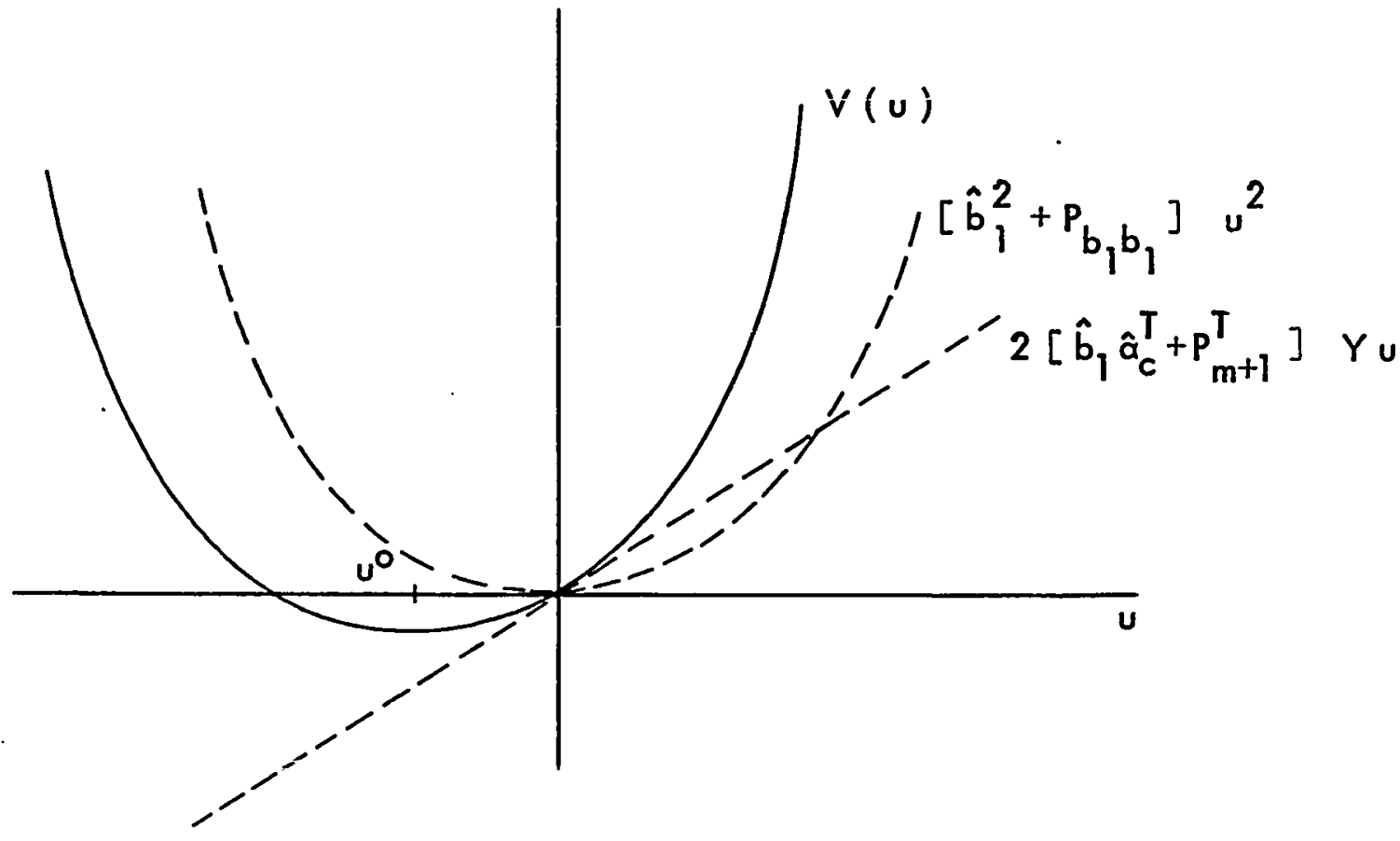

FIGURE 3.1 LOSS FUNCTION PART, DEPENDING ON $u(k)$ 
In Figure $3.1 u^{\circ}(k)$ is the adaptive non dual optimal control found earlier. It is seen that in order to keep the loss function $V(u)$ as low as possible while observing the constraint (3.41), we must choose the control

$$
u^{*}(k)=\bar{u}(k) \operatorname{sgn} u^{\circ}(k) \text { if } \bar{u}(k)>\left|u^{\circ}(k)\right|
$$

and

$$
u^{*}(k)=u^{0}(k) \text { if } \bar{\varphi}(k) \leq\left|u^{0}(k)\right|
$$

where

$$
\operatorname{sgn} c=\left\{\begin{array}{r}
1 \text { if } c>0 \\
-1 \text { if } c<0
\end{array}\right.
$$

The trace constraint (3.39) implies by (3.41) and (3.42) a minimal value for the control at each stage. Any control value larger than this minimum value $\bar{u}(k)$, is better from the point of view of information. Thus if the minimizing control, from the loss function point of view, is larger than the minimum $\bar{u}(k)$ we just use it, since it will give us more information in any event, with the best control at the same time, i.e., it will make $\operatorname{tr}^{-1}(k+1 \mid k+1)$ larger than $\bar{\rho}(k+1)$ and will provide the best control simultaneously.

We assumed above that the square root in $(3.42)$ is a real number. If it is not so, it means that $\operatorname{tr} P^{-1}(k+1 \mid k+1)$ is larger than $\bar{\rho}(k+1)$, even with no control at all. This simply means that the identification is good and the information measure is large enough without the weight of $u(k)$ in the observation $y(k+1)$. 
Thus we take,

$$
\bar{u}(k)=0 \text { if } \mu(k)<0
$$

where

$$
\mu(k)=R\left[\bar{\rho}(k+1)-\operatorname{tr} P^{\cdot 1}(k+1 \mid k)\right]-Y^{\top}(k) Y(k)
$$

We may now summarize the foregoing in a theorem.

Theorem 3.2: Dual adaptive control law.

For the system given by equations (3.5) and (3.7), let the admissible control $u(k)$ be a function of past inputs and outputs, up to stage $k$. Then the control law that minimizes the cost function,

$$
V=E\left[y^{2}(k+1) \mid y_{k}\right]
$$

subject to the constraint

$$
\operatorname{tr} P^{-1}(k+1 \mid k+1) \geq \bar{\rho}(k+1)
$$

with $\bar{\rho}(k+1)$ predetermined, is given by

$$
u^{*}(k)= \begin{cases}\bar{u}(k) \operatorname{sgn} u^{\circ}(k) & \text { if }\left|u^{\circ}(k)\right|<\bar{u}(k) \\ u^{\circ}(k) & \text { if }\left|u^{\circ}(k)\right| \geq \bar{u}(k)\end{cases}
$$

where

$$
u^{o}(k)=\frac{\left.\hat{b}_{1}(k+1 \mid k) \hat{a}_{c}(k+1 \mid k)+P_{n+1}(k+1 \mid k)\right]^{T} Y(k)}{b_{1}^{2}(k+1 \mid k)+P_{b_{1} b_{1}}(k+1 \mid k)}
$$


is the non dual adaptive control law (3.22), and

$$
\bar{v}(k)=\left\{\begin{array}{cl}
{[\mu(k)]^{1 / 2}} & \text { if } \mu(k)>0 \\
0 & \text { if } \mu(k) \leq 0
\end{array}\right.
$$

where

$$
\mu(k)=\bar{R}\left[\bar{\rho}(k+1)-\operatorname{tr} P^{-1}(k+1 \mid k)\right]-Y^{\top}(k) Y(k)
$$

From (3.49) we note that disregarding the trace constraint (i.e., $\bar{u}(k) \equiv 0)$, the dual controller becomes identical to the non dual one, $u^{*}(k)=u^{0}(k)$.

As was said above the function $p(k+1)$ must be predetermined. This can only be done through system simulation and experimentation. Practical considerations of this aspect and applications are left for the next chapter. Of major importance is also the knowledge of the system and parameters noise statistics, without which the filter is not estimating optimally. This aspect is dealt with later, but here we bring forth an interesting property of the adaptive control laws (3.22) and (3.29), which is the controller invariancy under amplification of the system noise samples. This will be made clear in the sequel.

Theorem 3.3:

For the system given by (3.5) and (3.7) and the dual control law (3.49), the parameter estimates and error covariance, are invariant under amplification of the system noise sample paths. Moreover, if the noise is amplified by a factor $c$ then 
the resulting control and loss function are likewise amplified by $c$ and $c^{2}$ respectively.

Proof:

The proof follows by induction. We assume that the theorem holds for some stage $i(e . g . i=1)$, and show that if then holds for stage $i+1$.

Combine equations $(3.26)$ and $(3.27)$

$$
\begin{aligned}
P(i+1 \mid i+1) & =P(i+1 \mid i)-P(i+1 \mid i) \varphi^{\top}(i) \\
& \cdot\left[\varphi(i) P(i+1 \mid i) \varphi^{\top}(i)+R\right]^{-1} \varphi(i) P(i+1 \mid i)
\end{aligned}
$$

and by $(3.24)$ and $(3.6)$

$$
\begin{gathered}
P(i+1 \mid i)=P(i \mid i)+Q \\
\varphi(i)=[y(i) y(i-1) \ldots y(i-n+1) \cup(i) \cup(i-1) \ldots u(i-n+1)]
\end{gathered}
$$

We denote all variables correponding to the case where the noise sample is amplified by factor $c$, with superscript $c, e . g ., u^{c}(k), \hat{x}^{c}(i)$, etc. Hence,

$$
R^{c}=c^{2} R
$$

By assumption and (3.54)

$$
P^{c}(i \mid i)=P(i \mid i), P^{c}(i+1 \mid i)=P(i+1 \mid i)
$$




$$
\varphi^{c}(i)=c \varphi(i)
$$

equation (3.53) yields using (3.56) - (3.58)

$$
\begin{aligned}
P^{c}(i+1 \mid i+1) & =P^{c}(i+1 \mid i)-P^{c}(i+1 \mid i) \varphi^{c T}(i) \\
& \cdot\left[\varphi^{c}(i) P^{c}(i+1 \mid i) p^{c T}(i)+R^{c}\right]^{-1} \varphi^{c}(i) P^{c}(i+1 \mid i) \\
& =P(i+1 \mid i)-P(i+1 \mid i) c \varphi^{\top}(i) \\
& \cdot[c \varphi(i) P(i+1 \mid i) p \\
& =P(i+1 \mid i)-c^{2} c^{-2} P(i+1 \mid i) D^{\top}(i) \\
& \cdot\left[\varphi(i) P(i+1 \mid i) \varphi^{\top}(i)+R\right]^{-1} \varphi(i) P(i+1 \mid i) \\
& =P(i+1 \mid i+1)
\end{aligned}
$$

By definition,

$$
v^{c}(i+1)=c v(i+1)
$$

and by assumption and (3.23)

$$
\hat{x}^{c} \cdot(i \mid i)=\hat{x}(i \mid i), \hat{x}^{c}(i+1 \mid i)=\hat{x}(i+1 \mid i)
$$

From (3.5) and using (3.58) obtain

$$
\begin{aligned}
y^{c}(i+1) & =\varphi^{c}(i) x^{c}(i+1)+v^{c}(i+1) \\
& =c \varphi(i) \times(i+1)+c v(i+1) \\
& =c y(i+1)
\end{aligned}
$$


using the fact that $x^{c}(i+1)=\times(i+1)$.

Rewrite equation (3.25) using (3.27), and the above results

$$
\begin{aligned}
\hat{x}^{c}(i+1 \mid i+1)= & \hat{x}^{c}(i+1 \mid i)+P^{c}(i+1 \mid i) \varphi^{c T}(i) \\
& \cdot\left[\varphi^{c}(i) P^{c}(i+1 \mid i) \varphi^{c T}(i)+R\right]^{-1}\left[y^{c}(i+1)-\varphi^{c}(i) \hat{x}^{c}(i+1 \mid i)\right] \\
= & \hat{x}(i+1 \mid i)+P(i+1 \mid i) C \varphi^{T}(i) c^{-2} \\
& \cdot\left[\varphi(i) P(i+1 \mid i) \varphi^{T}(i)+R\right]^{-1} c[y(i+1)-\varphi(i) \hat{x}(i+1 \mid i)] \\
= & \hat{x}(i+1 \mid i+1)
\end{aligned}
$$

(3.59) and (3.63) prove the first part of the theorem, and from (3.62) it is seen immediately that

$$
V^{c}=E\left[y^{c}(i+1)\right]^{2}=E[c y(i+1)]^{2}=c^{2} V
$$

It thus remains to show that $u^{c}(i+1)=c u(i+1)$. We first show this for the non dual control. From (3.50)

$$
\begin{aligned}
u^{c o}(i+1) & =-\left[\hat{b}_{1}^{c}(i+1 \mid i)+P_{b_{1} b_{1}}^{c}(i+1 \mid i)^{-1}\right. \\
& \cdot\left[\hat{b}_{1}^{c}(i+1 \mid i) \hat{a}_{c}^{c}(i+1 \mid i)+P_{n+1}^{c}(i+1 \mid i]^{\top} Y^{c}(i+1)\right. \\
= & -\left[\hat{b}_{1}^{2}(i+1 \mid i)+P_{b_{1} b_{1}}(i+1 \mid i)\right]^{-1} \\
& \cdot\left[\hat{b}_{1}(i+1 \mid i) \hat{a}_{c}^{c}(i+1 \mid i)+P_{n+1}(i+1 \mid i)\right]^{\top} \subset Y(i+1) \\
& =c u^{0}(i+1)
\end{aligned}
$$


using (3.59), (3.62), (3.63) and the definition of $Y(i+1)$ as in (3.13).

Now from (3.52)

$$
\begin{aligned}
\mu^{c}(i+1) & =R^{c}\left[\rho^{c}(i+2)-\operatorname{tr} P^{c}(i+2 \mid i+1)\right]-Y^{c T}(i+1) Y^{c}(i+1) \\
& =c^{2}\left[\bar{\rho}(i+2)-\operatorname{tr} P^{-1}(i+2 \mid i+1)\right]-c^{2} Y^{\top}(i+1) Y(i+1) \\
& =c^{2} \mu(i+1) .
\end{aligned}
$$

and thus by $(3.51)$

$$
\begin{aligned}
\bar{v}^{c}(i+1) & =\left\{\begin{array}{cl}
c[\mu(i+1)]^{1 / 2} & \text { if } c \mu(i+1)>0 \\
0 & \text { otherwise }
\end{array}\right. \\
& =c \bar{u}(i+1)
\end{aligned}
$$

and using (3.65) and (3.67) in (3.49), we finally prove for the dual control law

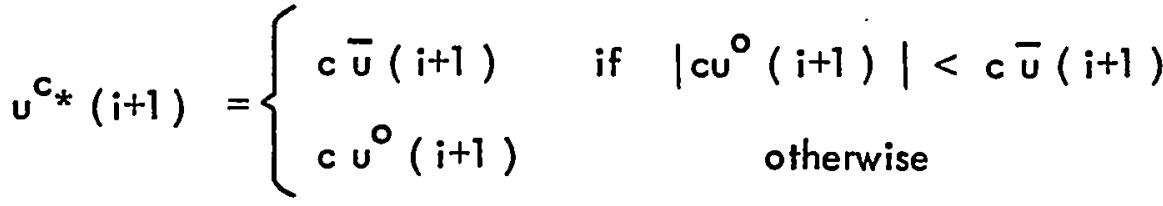

$$
\begin{aligned}
& =c u^{*}(i+1)
\end{aligned}
$$

We must now show that the theorem holds for $i=1$. Assume the initial conditions

$$
\begin{aligned}
& y(k)=0, u(k)=0 ; k \leq 0 \\
& \hat{x}(0)=\bar{x}_{0}, P(0)=P_{0} ; x(0)=x_{0}
\end{aligned}
$$


Now

$$
\begin{aligned}
y^{c}(1)=p(0) x_{0}+v^{c}(1) & =c v(1) \\
& =c y(1)
\end{aligned}
$$

since

$$
\varphi^{c}(0)=\varphi(0)=0 \text {. }
$$

By (3.24)

$$
\begin{aligned}
P^{c}(1 \mid 0)=P^{c}(0 \mid 0)+Q & =P_{0}+Q \\
& =P(1 \mid 0)
\end{aligned}
$$

and thus by (3.52)

$$
\mu^{c}(1)=c^{2} \mu(1)
$$

since $R^{c}=c^{2} R$ and $Y^{c}(1)=c Y(1)$. This in turn points to (by (3.51))

$$
\bar{u}^{c}(1)=c \bar{u}(1)
$$

and lastly in this line, by (3.49)

$$
u^{c} *(1)=c u^{*}(1)
$$

It is now easily verified by using the above results, and the filter equations ( 3.23$)$ (3.27) that

$$
\hat{x}^{c}(1 \mid 1)=\hat{x}(1 \mid 1) \text { and } P^{c}(1 \mid 1)=P(1 \mid 1)
$$


and this concludes the proof.

The implication of theorem 3.3 is the following. The system noise which is the noise in the observation, is of little importance to the adaptive control policy, assuming of course that this noise covariance $R$, is known. Whether this noise intensity is very low or very high, does not matter to the systems identification and control. This of course, is not the case with the parameter process noise intensity which has a direct bearing on the estimation error variances. In chapter 5 it is shown that the observation noise covariance $R$, is easily identified, using an adaptive filter in a system simulation with incomplete knowledge of noise covariances. The control performance depends on good identification which is not easy to accomplish with large noise covariance in the parameter process. But this performance is invariant under small or large observation noise. 


\section{CHAPTER IV}

\section{ADAPTIVE CONTROL SYSTEM SIMULATION}

\subsection{Introduction}

In this Chapter we investigate the performance of the dual adaptive control policy, as applied to several stochastic systems. The systems parameters are stochastic processes, with different driving"noise intensities, or deterministic unknown time functions. The model parameters are assumed to be random processes in every case, as was assumed in Chapter 3. The noise covariance of the parameter process is used in the control policy and must be known.

In some examples we assume exact knowledge of this covariance, and in others the actual value is assumed different from the value used.

The dual controller is compared with the non-dual control law in all the examples, and in some it is also compared with the perturbation control policy [3]- [5]. For the purpose of comparison we use the accumulated loss function

$$
L(k)=\sum_{i=1}^{k} y^{2}(i)
$$

All the examples but two, deal with first order systems, i.e., systems with first order dynamics, containing two unknown parameters, one of which is the gain $b_{1}$. Two examples are concerned with a second order system, having three random parameters. The simulation was done on an IBM 360 model 75 computer. 


\subsection{First-Order System Simulations}

The systems simulated here are of first-order, and have two unknown parameters. Thus the input-output representation of the model is

$$
y(k)=a(k) y(k-1)+b(k) u(k-1)+v(k)
$$

or in the form of equations (3.5), (3.7)

$$
\begin{aligned}
& y(k)=\varphi(k-1) x(k)+v(k) \\
& x(k+1)=x(k)+\omega(k)
\end{aligned}
$$

where the state representation of the parameter process is of second order, and

$$
\begin{aligned}
& \varphi(k-1) \approx\left[\begin{array}{ll}
y(k-1) & u(k-1)
\end{array}\right] \\
& x(k)=\left[\begin{array}{ll}
a(k) & b(k)
\end{array}\right]^{\top} \\
& \omega(k)=\left[\begin{array}{ll}
\omega_{a}(k) & \omega_{b}(k)
\end{array}\right]^{\top}
\end{aligned}
$$

and where

$$
\begin{aligned}
& v(k) \sim N[0, R] \\
& \omega(k) \sim N[0, Q] \\
& Q=\operatorname{diag}\left[\begin{array}{ll}
q_{a} & q_{b}
\end{array}\right]
\end{aligned}
$$

For this model the non-dual adaptive control law, Equation (3.22), is

$$
u^{0}(k)=-\left[\hat{b}^{2}(k+1 \mid k)+P_{b b}(k+1 \mid k)\right]^{-1}\left[\hat{b}(k+i \mid k) \hat{a}(k+1 \mid k)+P_{a b}(k+1 \mid k)\right] y(k)
$$


since here by $(3.12),(3.13)$,

$$
\begin{aligned}
& \hat{a}_{g}(k+1 \mid k)=\left[\begin{array}{lll}
\hat{a}(k+1 \mid k) & 0
\end{array}\right]^{\top} \\
& Y(k)=\left[\begin{array}{lll}
y(k) & 0
\end{array}\right]^{\top}
\end{aligned}
$$

and by definition

$$
\therefore P=\left[\begin{array}{cc}
P_{a a} & P_{a b} \\
P_{a b} & P_{b b}
\end{array}\right] \quad P_{n+1}=P_{2}^{-}=\left[\begin{array}{ll}
P_{a b} & P_{b b}
\end{array}\right]^{\top}
$$

The perturbation controller, Equation (3.28) is

$$
u^{P}(k)=u^{0}(k)+(-1)^{k} \cdot \delta
$$

and the dual control law is by (3.49) - (3.52)

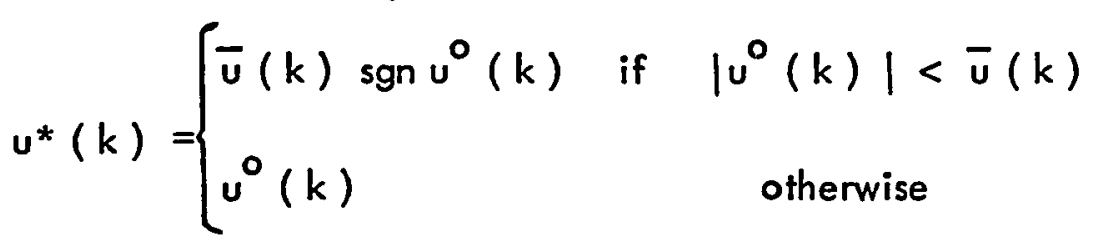

$$
\begin{aligned}
& \bar{v}(k)=\left\{\begin{array}{cc}
\sqrt{\mu}(k) & \text { if } \mu(k)>0 \\
0 & \text { otherwise }
\end{array}\right. \\
& \mu(k)=R\left[\bar{\rho}(k+1)-\operatorname{tr} P^{-1}(k+1 \mid k)\right]-y^{2}(k)
\end{aligned}
$$

The Filter equations are by $(3.23)-(3,27)$

$$
\hat{x}(k+1 \mid k)=\hat{x}(k \mid k-1)+K(k)[y(k)-\varphi(k-1) \hat{x}((k \mid k-1)](4.19)
$$




$$
\begin{aligned}
& K(k)=P(k \mid k-1) \varphi^{\top}(k-1)\left[\varphi(k-1) P(k \mid k-1) \varphi^{\top}(k-1)+R\right]^{-1}(4.20) \\
& P(k+1 \mid k)= {[I-K(k) \varphi(k-1)] P(k \mid k-1)[I-K(k) \varphi(k-1)]^{\top} } \\
&+K(k) P(k \mid k+1) K(k)+Q
\end{aligned}
$$

Equation (4.21) is used for the error covariance matrix computation, because in this form $P$ is always updated as a positive definite matrix, even if the gain is computed in error, due to computer round off. 'It is important for the filter stability that $P$ is positive definite $[13]$.

It should be noted that the error covariance update as in (4.21), is more time consuming than the conventional method of equation $(3.26)$, but its numerical precision is better [ 15$]$.

The trace of the covariance matrix inverse, is needed for the dual controller and is given in the present case very simply as

$$
\operatorname{tr} P^{-1}=\frac{\operatorname{tr} P}{\operatorname{det} P}
$$

In its simplest form, the controller uses for $\bar{p}(k+1)$ a constant

$$
\bar{\rho}(k+1)=\rho_{c}
$$

this form requires the minimum amount of computation, and the implementation is the simplest. There are however two shortcomings of this form. In the first instance since at the beginning of control action we have very little information, we must assume large initial error variances, which means that $\operatorname{tr} \mathrm{P}^{-1}$ is small, and thus the controller produces large signals, whose function is to improve quickly the parameter 
estimates. But the loss function at the same time increases sharply as the control is bad. If however we argue that at steady state we don't care about the poor initial control, then it is obvious that employing this kind of control, the transient initial period of much uncertainty, becomes very short, and the filter "catches ori" fast. It was found that if $\rho_{c}$ is designed as in Figure 4.1 then it is possible to shorten the filter transient period, and to prevent very large control signals at the same time. In the initial period up to $t_{1}, \rho_{c}$ is increased evenly, as more information is

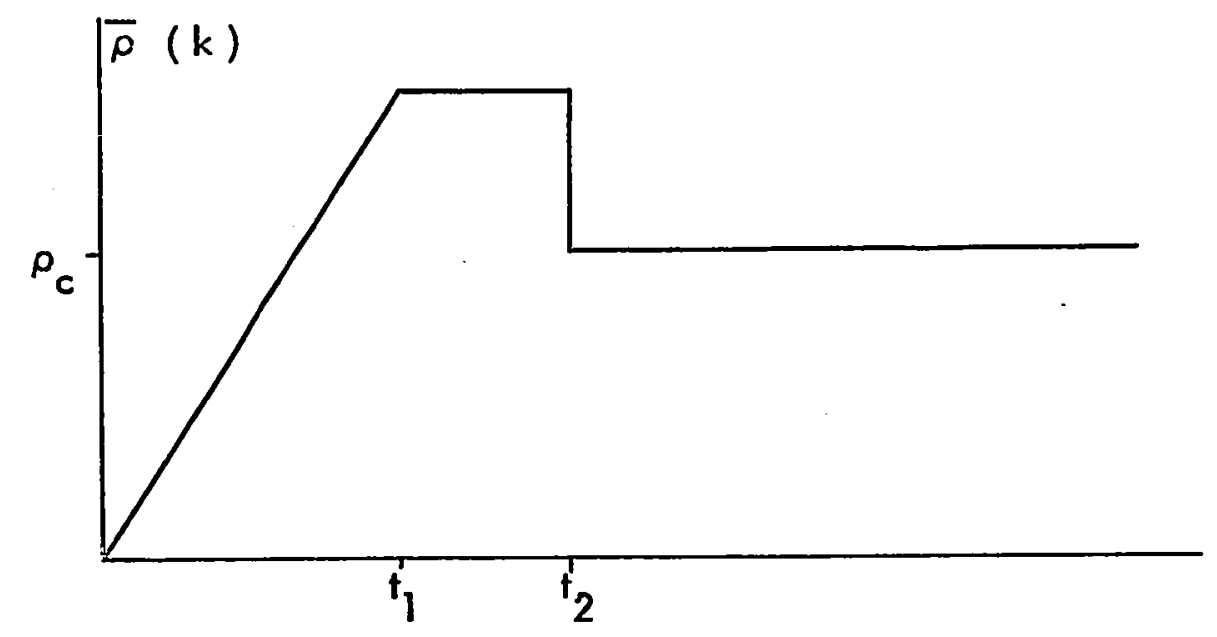

FIGURE 4.1 A DESIGN FOR THE TRACE CONSTRAINT

obtained by the filter. At the interval $\left[t_{1}, t_{2}\right] \rho_{c}$ is kept constant to ensure that the information does not decrease. At stage $t_{2}$ the filter is assumed to be already in steady state and from then on more attention is given to the control part, assuming that the filter is capable of tracking the parameters without much interference. Thus $\rho_{c}$ is decreased and kept a constant, so that the controller will interfere with the 
filtering part, only when the information measure decreases sharply.

The determination of $\bar{p}(k+1)$ as in Figure 4.1 still makes it a constant (except for a short transient period), and the controller depends strongly on the $\operatorname{tr} P^{-1}(k+1 \mid k)$ function as is seen from Equation (4.18). This was found unsatisfactory in some cases or at least, given to improvement. It was observed during system simulations that the controller using a constant $\bar{\rho}(k+1)$, was slow in many cases, to detect the turn-off phenomenon, and take the necessary action to end it. The reason is that because the $\operatorname{tr}^{-1}$ is a nonlinear function of the different parameter error variances, it is possible that one of these variances will increase appreciably, without decreasing the $\operatorname{tr} \mathrm{P}^{-1}$ function significantly. From the relation

$$
\operatorname{tr} P^{-1}=\frac{P_{a a}+P_{b b}}{P_{a a b b} P_{a b}-P_{a b}^{2}}=\frac{P_{a a}+P_{b b}}{\operatorname{det} P}
$$

it is seen that $\operatorname{tr}^{-1}$ is a function of the denominator difference which is the $P$ matrix determinant. The inverse trace may remain quite large even when $P_{b b}$ increases, because the determinant may remain small.

During the turn-off phenomenon the filter loses track of the gain parameter $b(k)$, and the error variance $P_{b b}$ thus increases. But the $\operatorname{tr} P^{-1}$ may not decrease, and may even increase for some length of time. The filter later adjusts $\mathrm{P}^{-1}$ to the information loss, and $\operatorname{tr}^{-1}$ eventually decreases. It is also observed that during the furn-off state, only the parameter $a(k)$ is being 
effectively identified since from the observation equation

$$
y(k+1)=a(k+1) y(k)+b(k+1) u(k)+e(k+1)
$$

it is seen that without the $u(k)$ signal, the filter receives information only about $a(k+1)$ and thus it has more confitence in its estimate. Under these conditions we have

$$
P_{b b} \gg P_{a a} \cdot
$$

and (4.24) is approximated as

$$
\operatorname{tr} \mathrm{P}^{-1} \cong \frac{\mathrm{P}_{b b}}{\mathrm{P}_{a a_{b b}} \mathrm{P}_{b b}}=\frac{1}{\mathrm{P}_{a a}}
$$

assuming that

$$
P_{a a} P_{b b} \gg P_{a b}^{2}
$$

We now argue that we would like to have approximately the situation, in which

$$
P_{a d} \cong P_{b b}
$$

then (4.24) becomes

$$
\operatorname{tr} P^{-1} \cong \frac{2 P_{a d}}{P_{a d}^{2}}=\frac{2}{P_{a d}}
$$

Comparing (4.26) and (4.29), we modify the dual controller to improve its ability to defect the information loss about the gain b, by modifying $\bar{\rho}(k+1)$ of Equation (4.23) to be 


$$
\bar{\rho}(k+1)=\left\{\begin{array}{cll}
P_{c} & \text { if } & P_{b b}(k+1 \mid k) \leq P_{b b}^{M} \\
\frac{a_{b}}{P_{a a}(k+1 \mid k)} \text { if } & P_{b b}(k+1 \mid k)>P_{b b}^{M}
\end{array}\right.
$$

where $P_{b b}^{M}$ is a fixed value which is sort of upper bound for $P_{b b}(k+1 \mid k)$, over which $\bar{\rho}(k+1)$ departs from its constant value $\rho_{c}$, and $\alpha_{b}$ is fixed in the range $1<q_{b}<2$. Again, these two values must be determined by experimentation through simulation. The value of $P_{b b}^{M}$ determines how closely $P_{b b}(k+1 \mid k)$ is being tracked separately, in order to be kept below a certain level.

This intuitive technique is not quite possible for higher order systems, but a simpler way is just to leave $\bar{\rho}(k+1)$ as a constant, and change $\bar{u}(k)$ of equation ( 4.17 ) as follows

$$
\bar{u}(k)=\left\{\begin{array}{cll}
\sqrt{\mu}(k) & \text { if } & \mu(k)>0 \\
0 & \text { if } & \mu(k) \leq 0 \text { and } P_{b b}(k+1 \mid k) \leq P_{b b}^{M} \\
u^{M} & \text { if } & \mu(k) \leq 0 \text { and } P_{b b}(k+1 \mid k)>P_{b b}^{M}
\end{array}\right.
$$

where $u^{M}$ is a constant. This technique simply forces a relatively large control signal when the gain parameter estimation error reaches above a certain value, thus keeping a good estimate of the gain parameter and preventing the turn-off phenomenon right at its start.

These modifications of the dual controller are not necessary at all, if we choose $\rho_{c}$ (the constant value of $\bar{\rho}(k+1)$ ) large enough. But then, together 
with very good estimation, there will be a cerfain degradation of the control, and a resulting increase in the loss function.

We next derive the lowest possible average loss-funetion. For this purpose we derive the expected loss for the minimum variance policy of a system with known parameters. Thus the system is given by

$$
\begin{aligned}
y(k)= & a_{1} y(k-1)+a_{2} y(k-2)+\ldots+a_{n} y(k-n) \\
& +b_{1} u(k-1)+b_{2} u(k-2)+\ldots+b_{n} u(k-n)+v(k)
\end{aligned}
$$

The expected loss is

$$
\begin{aligned}
& E[V(k+1)]=E\left[y^{2}(k+1)\right] \\
&=E\left[\left(a_{1} y(k)+\ldots+a_{n} y(k-n+1)+b_{2} u(k-1)\right.\right. \\
&+\ldots+b_{n} u(k-n+1)+b_{1} u(k) \\
&\left.+v(k+1))^{2}\right]
\end{aligned}
$$

differentiating (4.33) with respect to $u(k)$ and equating to zero

$$
\begin{aligned}
& 2 b_{1}^{2} u(k)+2 b_{1}\left[a_{1} y(k)+\ldots+a_{n} y(k-n+1)+b_{2} u(k-1)\right. \\
& \left.+\ldots+b_{n} u(k-n+1)\right]=0
\end{aligned}
$$

yields the minimizing control

$$
\begin{aligned}
u_{\min }(k)= & -\frac{1}{b_{1}}\left[a_{1} y(k)+\ldots+a_{n} y(k-n+1)\right. \\
& +b_{2} u(k-1)+\ldots+b_{n} u(k-n+1)
\end{aligned}
$$


To find the optimal loss, substitute $(4.35)$ in $(4.33)$

$$
E\left[V_{\min }(k+1)\right]=E\left[v^{2}(k+1)\right]=R
$$

and the optimal accumulated expected loss is

$$
E\left[L_{\min }(k)\right]=\sum_{i=1}^{k} E\left[V_{\min }(k)\right]=k R
$$

The average execution time for the dual controller system simulation

was about $4.7 \mathrm{msec}$. per stage, on the IBM 360 model 75 computer.

\subsubsection{Example 1. Average Performance Comparison}

In this example the non-dual, dual and perturbation controllers are compared, by obtaining their respective average accumulated loss functions. This is done by averaging the loss over fifty runs, using different randomly selected noise samples. The initial state vector $\times(0)$ (parameters), is taken randomly from a normal distribution,

$$
\begin{aligned}
& N\left[\gamma_{0}, \Gamma_{0}\right] \text { where } \\
& \gamma_{0}=\left[\begin{array}{ll}
0.7 & 1.5
\end{array}\right]^{\top} \\
& \Gamma_{0}=\operatorname{diag}\left[\begin{array}{ll}
0.01 & 0.1
\end{array}\right]
\end{aligned}
$$

The initial parameters vector estimate is

$$
\hat{x}(1 \mid 0)=\left[\begin{array}{l}
\hat{a}(1 \mid 0) \\
\hat{b}(1 \mid 0)
\end{array}\right]=\left[\begin{array}{l}
0.1 \\
0.1
\end{array}\right]
$$


and the initial error covariance is taken as,

$$
P(1 \mid 0)=\operatorname{diag}\left[\begin{array}{ll}
4.0 & 4.0
\end{array}\right]
$$

reflecting high uncertainty in $\hat{x}(1 \mid 0)$.

The noise data is,

$$
\begin{aligned}
& R=0.04 \\
& Q=\operatorname{diag}\left[\begin{array}{ll}
q_{a} & q_{b}
\end{array}\right]=\operatorname{dieg}\left[\begin{array}{lll}
0.0002 & 0.02
\end{array}\right]
\end{aligned}
$$

The dual controller used $\bar{\rho}(k+1)$ as in equation (4.30) with

the parameters,

$$
\begin{array}{ll}
\rho_{c}=20 . & P_{b b}^{M}=2 . \\
a_{b}=1.2 &
\end{array}
$$

and the periurbation controller used a square wave with amplitude (Equation (4.15))

$$
\delta=0.02
$$

These constants where found to yield best results by simulation.

The three control policies performances, are compared in Figure 4.2, which shows the average accumulated loss curves of Equation (4.1).

Figure 4.3 shows the same curves as Figure 4.2 but with the loss of the initial 50 stages eliminated i.e., $L(k)-L(50)$ where $L(k)$ is the average accumulated loss at stage $k$. 


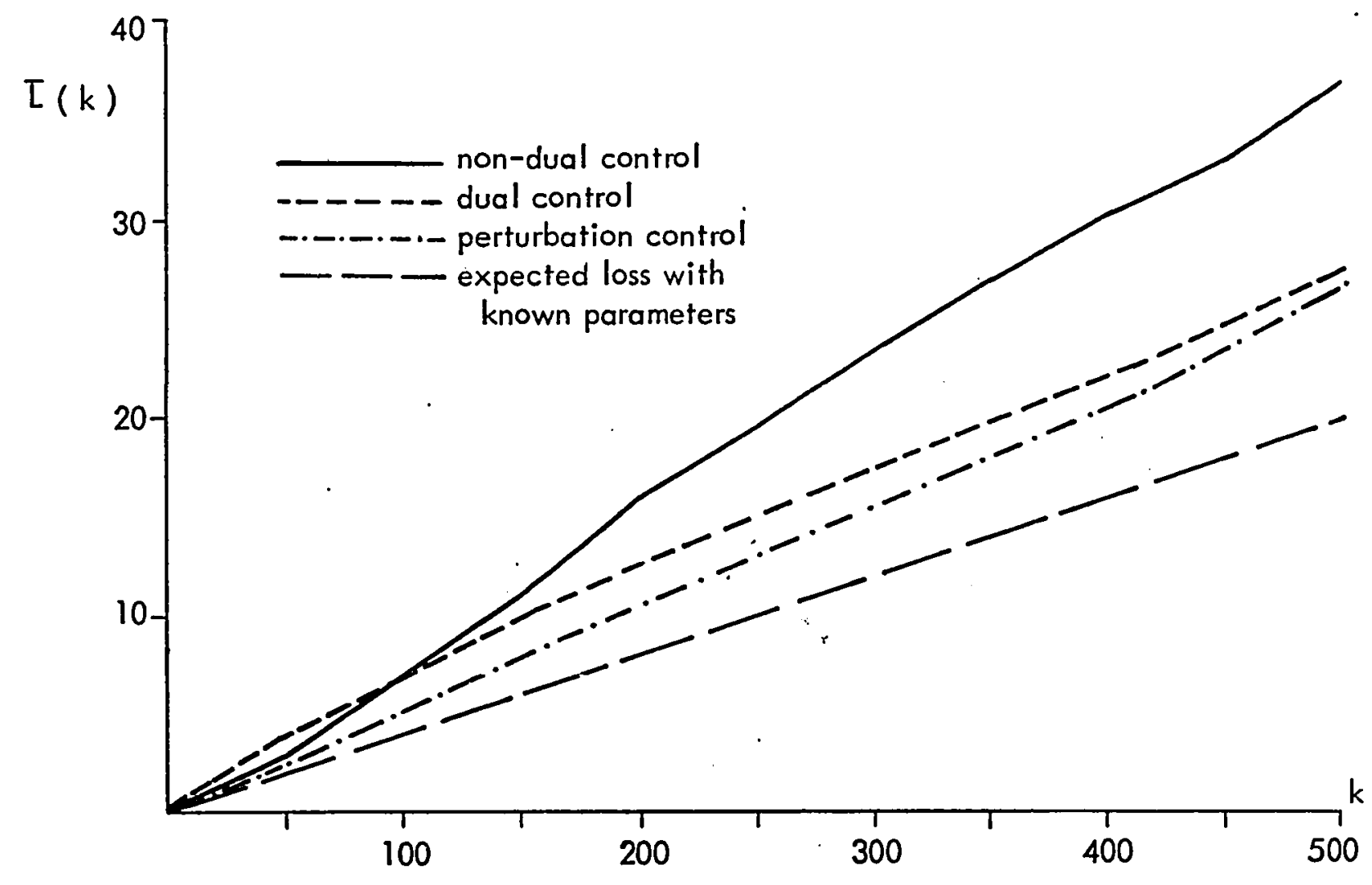

FIGURE 4.2 AVERAGE ACCUMULATED LOSS FUNCTIONS, EXAMPLE 1.

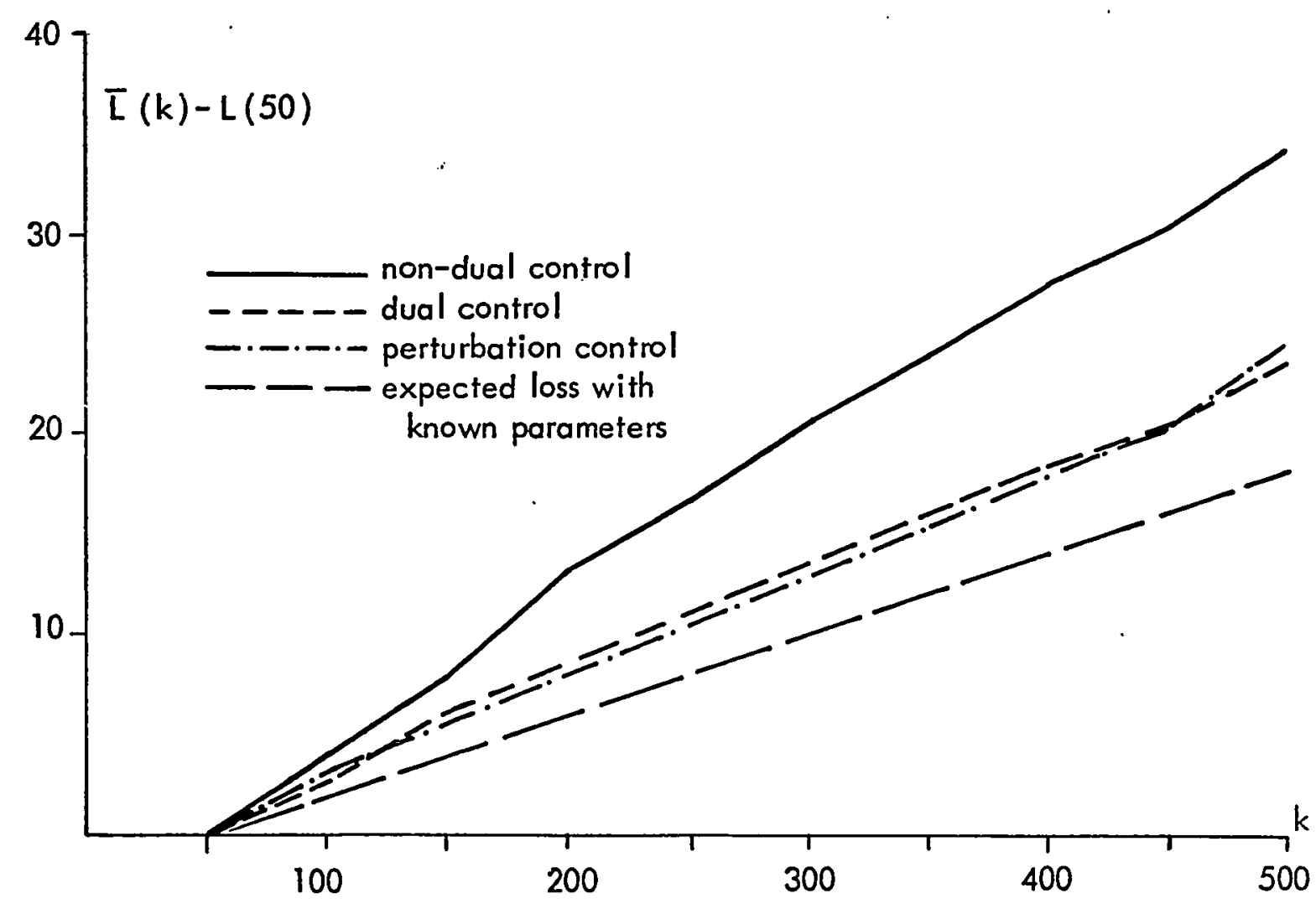

FIGURE 4.3 NORMALIZED AVERAGE ACCUMULATED LOSS FUNCTIONS, EXAMPLE 1. 
Comparing the results described in Figures 4.2 and 4.3, we can conclude that the dual and perturbation controller are about equal and superior to the non-dual control policy.

\subsubsection{Example 2. Single Run Results.}

In this example we investigate one run of the 50 runs batch, that was used in Example 1. The simulation datá and parameters are the same as in Example 1. We wish here to study in more detail the differences in the operation of the dual and non-dual adaptive control laws.

Figure 4.4 compares the accumulated loss functions of the two control policies. Since the performance depends directly on the quality of identification, we show in Figure 4.6 the random gain parameter time evolution $b(k)$, and its estimates $\hat{b}(k)$, using the two controllers. Figure 4.7 describes the gain parameter error covariance $P_{b b}(k)$, and Figure 4.5 shows the parameter a (k) sample path, as well as its estimates $\hat{a}(k)$, under the two control strategies.

From Figure 4.4 we observe that the two controllers are almost identical in their performance, up to stage 180. Very different in the interval 180 to 350 approximately, and equal again from stage 350 onward, as the slopes of the two loss functions are about the same. In the interval 180 to 350 we see first, that the slope of the non-dual controller loss function is greater than that of the dual one, and has a sharp jump in the interval 320 to 350 . 
The reason for the deterioration in performance under the non-dual policy is seen in Figure 4.6, where the turn-off phenomenon is observed. The gain parameter $b(k)$ is identified as almost zero (the control signals are also almost zero). The rapid increase in the loss function starts at about stage 200, as from then on, the gain $b(k)$ is quite large and hence its estimation error is also large, and at the same time the parameter $a(k)$ is growing at a fast rate as is seen in Figure 4.5. At stage 320 a large jump in the loss function is seen. The reason for this is that the control signals being effectively zero and the fact that a $(k)$ is greater than one combine to make an unstable free system. Since there is no control, the uncontrolled system is

$$
y(k)=a(k) y(k-1)+v(k) ; a(k)>1
$$

an unstable system. This mode of control-turn-off, coupled with instability ends when the large output values $y(k)$, force an increase in the control values as is seen in Equation (4.11), where $y(k)$ becomes dominant. The increased control signals that occur around stage 340 improve drastically the estimate of $b(k)$, and $P_{b b}(k)$ reduces very sharply as is seen in Figures 4.6 and 4.7. The control turn-off thus ends and the system experiences good control.

The dual controller prevents the turn-off phenomenon by monitoring a measure of the error covariance and acts to maintain good identification.

The importance of good identification of the gain parameter is clear, as its absence leads to the turn-off of the controller. The dual controller in producing 


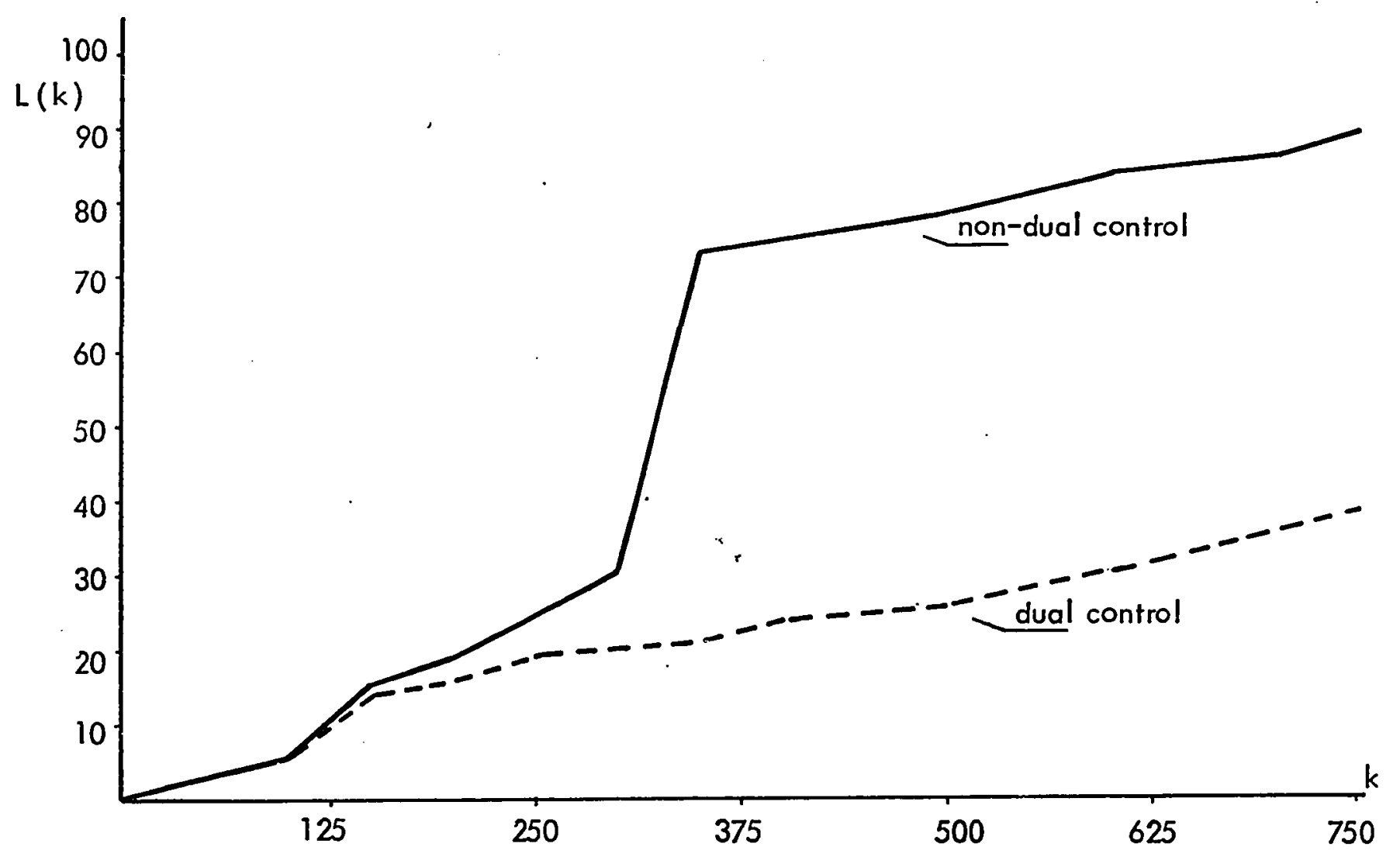

FIGURE 4.4 ACCUMULATED LOSS FUNCTIONS, EXAMPLE 2.

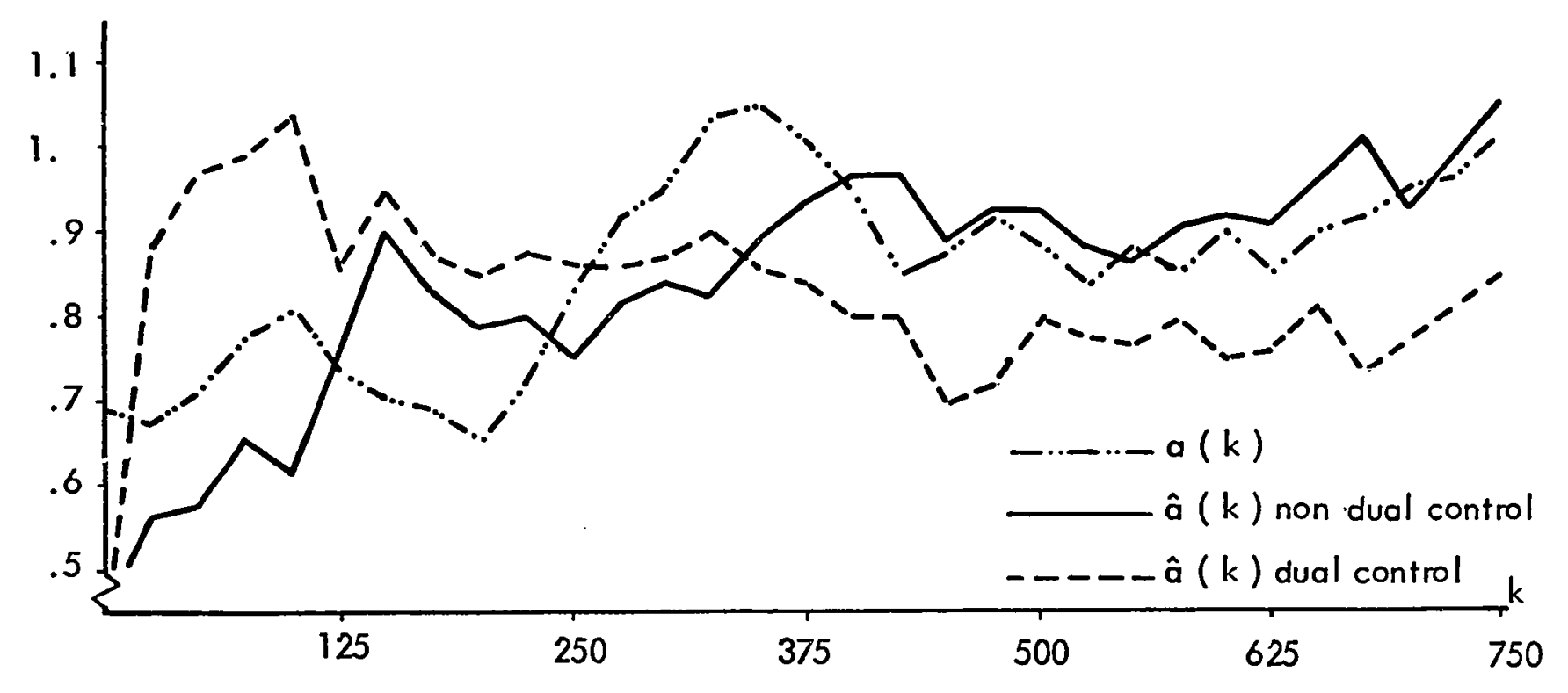

FIGURE 4.5 THE a $(k)$ PARAMETER AND ITS ESTIMATES, EXAMPLE 2. 


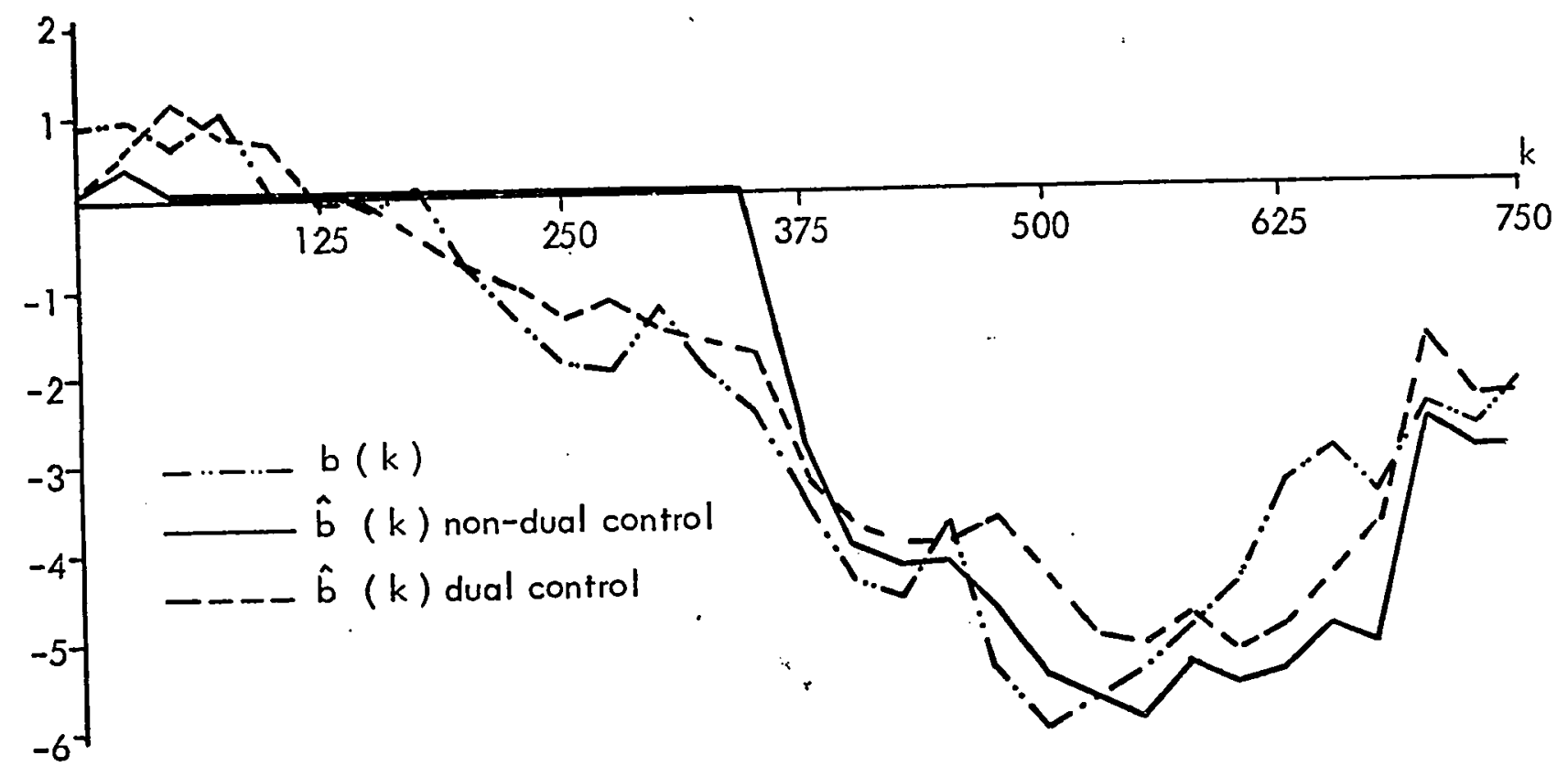

FIGURE 4.6 THE GAIN $b(k)$ AND ITS ESTIMATES, EXAMPLE 2.

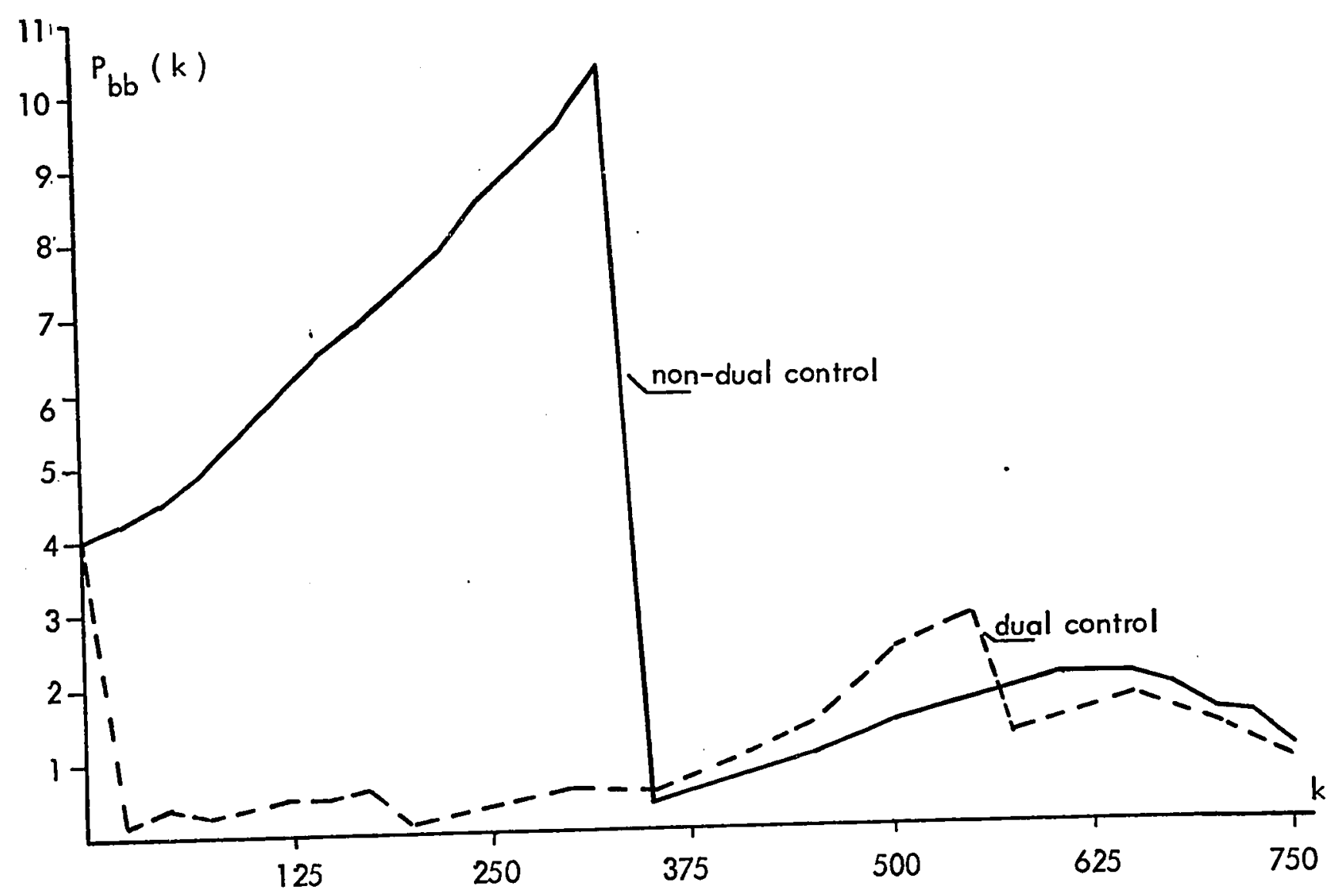

FIGURE 4.7 THE GAIN PARAMETER ERROR COVARIANCE, EXAMPLE 2. 
relatively large signals for information correction, brings about better identification of $b(k)$, but somewhat inferior identification of $a(k)$, in comparison to the non-dual controller action. The season is very simply the fact that the gain parameter is being weighted more importantly than $a(k)$ in the observation $y(k)$ when using the dual control policy. This can be observed in Figure 4.7. This fact however has a negligible influence on the resulting control signals. It is important to note that the relatively large control signals are not produced constantly but only when there is a reduction in the information available. Mostly the control signals are those needed for good control, as are produced by the non-dual controller.

\subsubsection{Example 3. Average Performance with Incomplete Knowledge of Noise Covariance.}

In this example we once more wish to compare the non-dual, dual and perturbation control policies, by obtaining the average accumulated lossfunctions in the three cases. Unlike Example I however, here we do not assume exact knowledge of the random parameters noise statistics. The actual naise covariance $Q$, is not known and an assumed value $Q_{\text {as }}$ is used in the filter. Under this condition the estimates are not optimal any more. It was shown in the literature $[2],[6]-[8]$, that if the assumed noise covariance is larger than the actual one, then the estimation error covariance, is bounded by the error covariance resulting from the assumed $Q_{a s}$. Note that $Q_{a s}$ is greater than $Q$ if $Q_{a s}-Q$ is positive definite. It thus seems logical to choose the unknown noise covariance, 
as larger than the actual value.

As in Example 1 we simulate 50 runs of the system using random noise samples, and random initial conditions of the parameters. We use the same values as in Example 1 for $x(0), \hat{x}(1 / 0)$ and $\rho(1 \mid 0)$, as is given below

$$
\begin{aligned}
& x(0) \sim N\left[\gamma_{0}, \Gamma_{0}\right]_{r} \\
& \gamma_{0}=\left[\begin{array}{ll}
0.7 & 1.5
\end{array}\right]^{\top} \\
& \Gamma_{0}=\operatorname{diag}\left[\begin{array}{lll}
0.01 & 0.1
\end{array}\right] \\
& \hat{x}(1 \mid 0)=\left[\begin{array}{ll}
1 & (1 \mid 0) \hat{b}(1 \mid 0)
\end{array}\right]^{\top}=\left[\begin{array}{lll}
0.1 & 0.1
\end{array}\right]^{\top} \\
& P(1 \mid 0)=\operatorname{diag}\left[\begin{array}{ll}
4.0 & 4.0
\end{array}\right]
\end{aligned}
$$

The noise parameters are

$$
\begin{aligned}
& R=0.04 \\
& Q_{a s}=\operatorname{diag}\left[a_{a} q_{b}\right]=\operatorname{diag}\left[\begin{array}{ll}
0.0002 & 0.02
\end{array}\right]
\end{aligned}
$$

the assumed covariance of the parameter process which is used in the filter equations.

The actual noise covariance is

$$
Q=\frac{1}{36} Q_{\text {as }}
$$

and this value is used in the plant simulation. 
The accumulated loss functions are averaged over the 50 runs in the three control cases, and are shown in Figure 4.8. The non-dual control loss curve exhibits a large rise in the interval of stage 400 to stage 450 approximately. This is the contribution of one particular run. Eliminating this run from the average calculation yields the loss curves of Figure 4.9.

\section{A comparison of the loss curves in Figures 4.8 and 4.9 clearly} indicates the superiority of the dual controller in this case where the noise covariance is not known exactly.

Since the assumed noise covariance $Q_{\text {as }}$ is the same as in Example 1, the dual and perturbation controllers used the same parameters that were found to yields best results in the system of Example 1. The dual controller parameters

$$
\rho_{c}=20 . \quad p_{b b}^{M}=2 . \quad a_{b}=1.2
$$

The perturbation square wave amplitude

$$
\delta=0.02 \text {. }
$$

Under these conditions the dual controller performed better than the perturbation controller. It should be noted however, that this conclusion is based solely on simulation and numerical experimentation of limited nature, and as such is not general. 


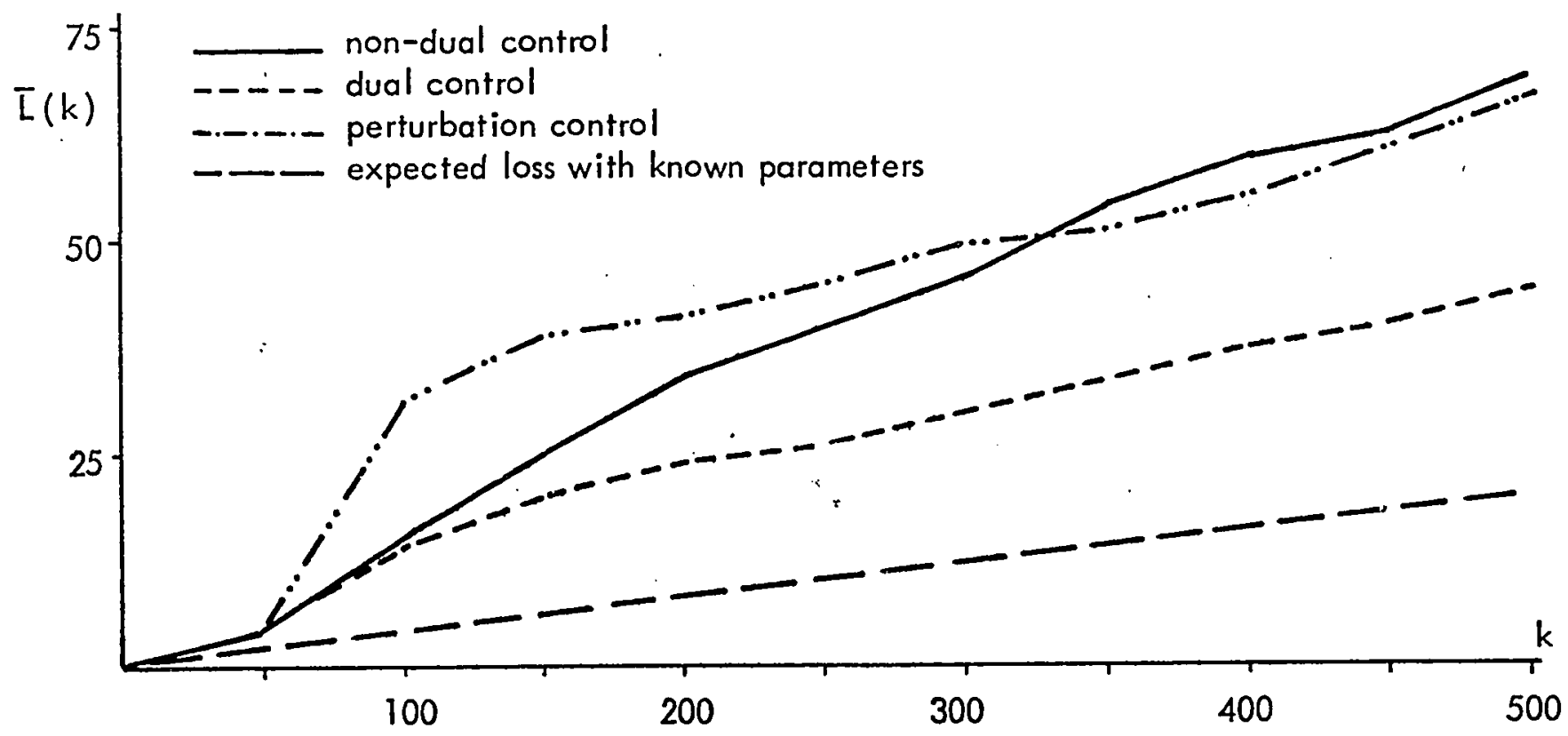

FIGURE 4.9 AVERAGE ACCUMULATED LOSS FUNCTIONS, EXAMPLE 3.

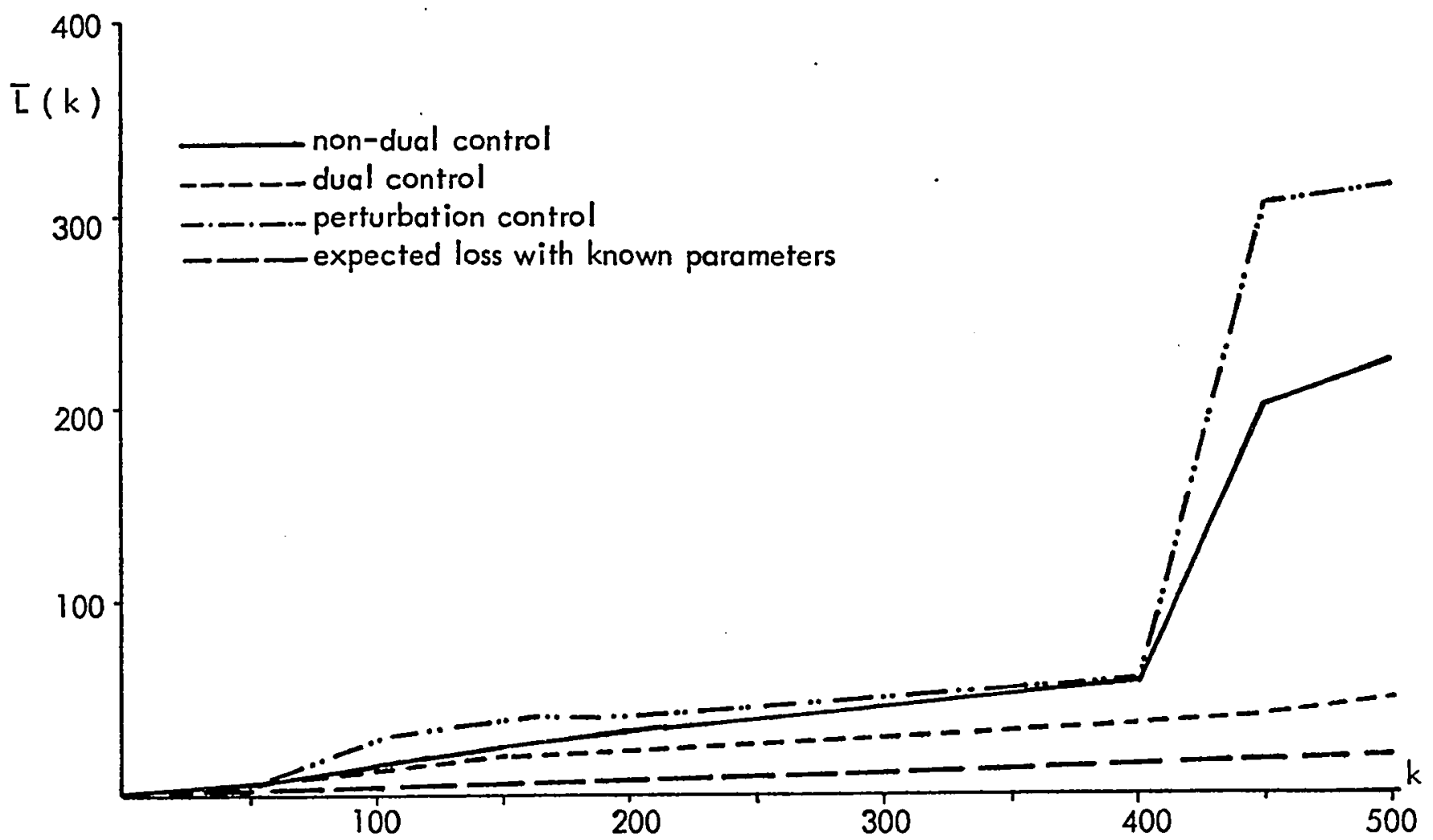

FIGURE 4.8 AVERAGE ACCUMULATED LOSS FUNCTIONS, EXAMPLE 3. 
4.2.4. Example 4 Single Run Results With Incomplete Knowledge of Noise

\section{Covariance.}

As was done in Example 2, we investigate here one run of the fifty

used in Example 3. The dual and non-dual control policies are compared, simulating the system with the same noise samples and initial conditions. The simulation

parameters are those given in Example 3. Results are shown in Figures 4.10-4.13. The accumulated loss curves are compared in Figure 4.11. Figure 4.10 shows the $a(k)$ parameter smaple path and its estimates $\hat{a}(k)$. The same information is given in Figure 4.12 for the gain parameter $b(k)$ and its estimates $\hat{b}(k)$, while Figure 4.13 describes the gain parameter error variances under the two control regimes.

The weak performance of the non-dual control policy for this case, where the parameters noise covariance is not exactly known, is clearly seen in Figure 4.11, which demonstrates a sharp rise in the loss function, starting around stage 100 and terminating around stage 160 . The reason for this is clear from Figures 4.10,4.12 and 4.13. The control is turned-off as is seen in Figure 4.12, where $\hat{b}(k)$ is practically zero in the interval $k \in[20,160]$. There follows a free system of the form

$$
y(k)=a(k) y(k-1)+v(k)
$$

but as long as $a(k)<1$ the loss is not very large. At stage 120 and over, the value of $a(k)$ is closer to, and even greater than 1 . The output values become 


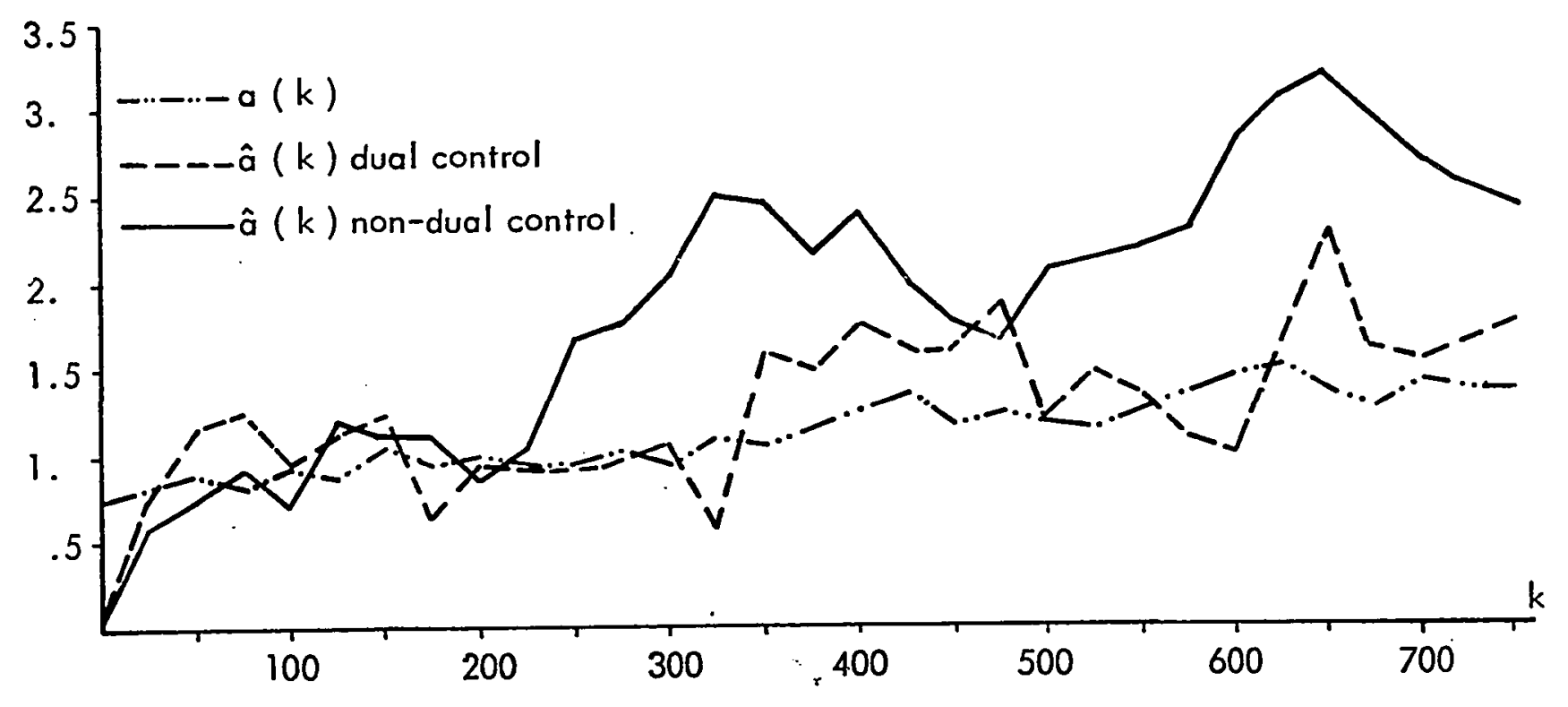

FIGURE 4.10 THE a ( $k$ ) PARAMETER AND ITS ESTIMATES, EXAMPLE 4.

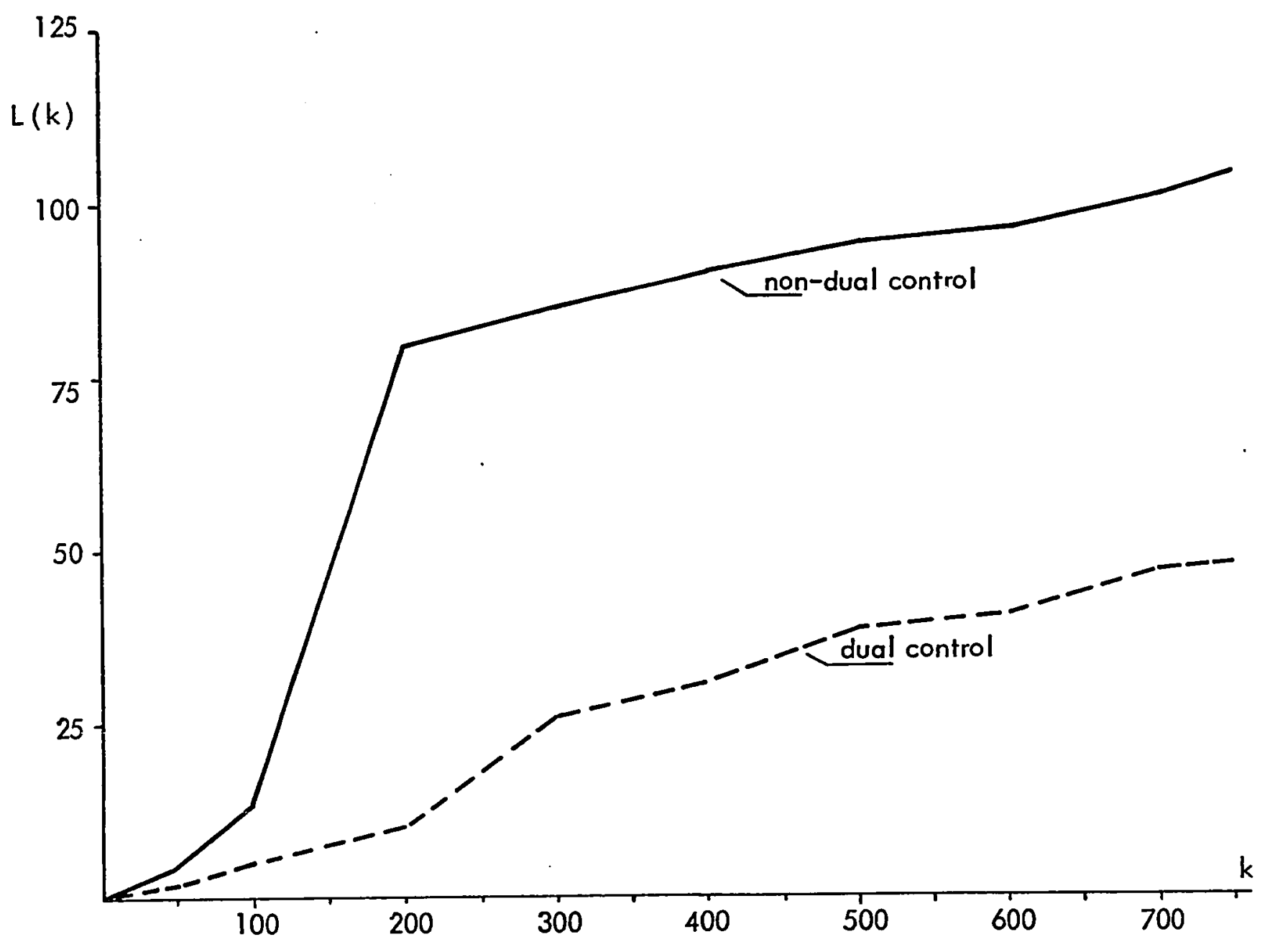

FIGURE 4.11 ACCUMULATED LOSS FUNCTIONS, EXAMPLE 4. 


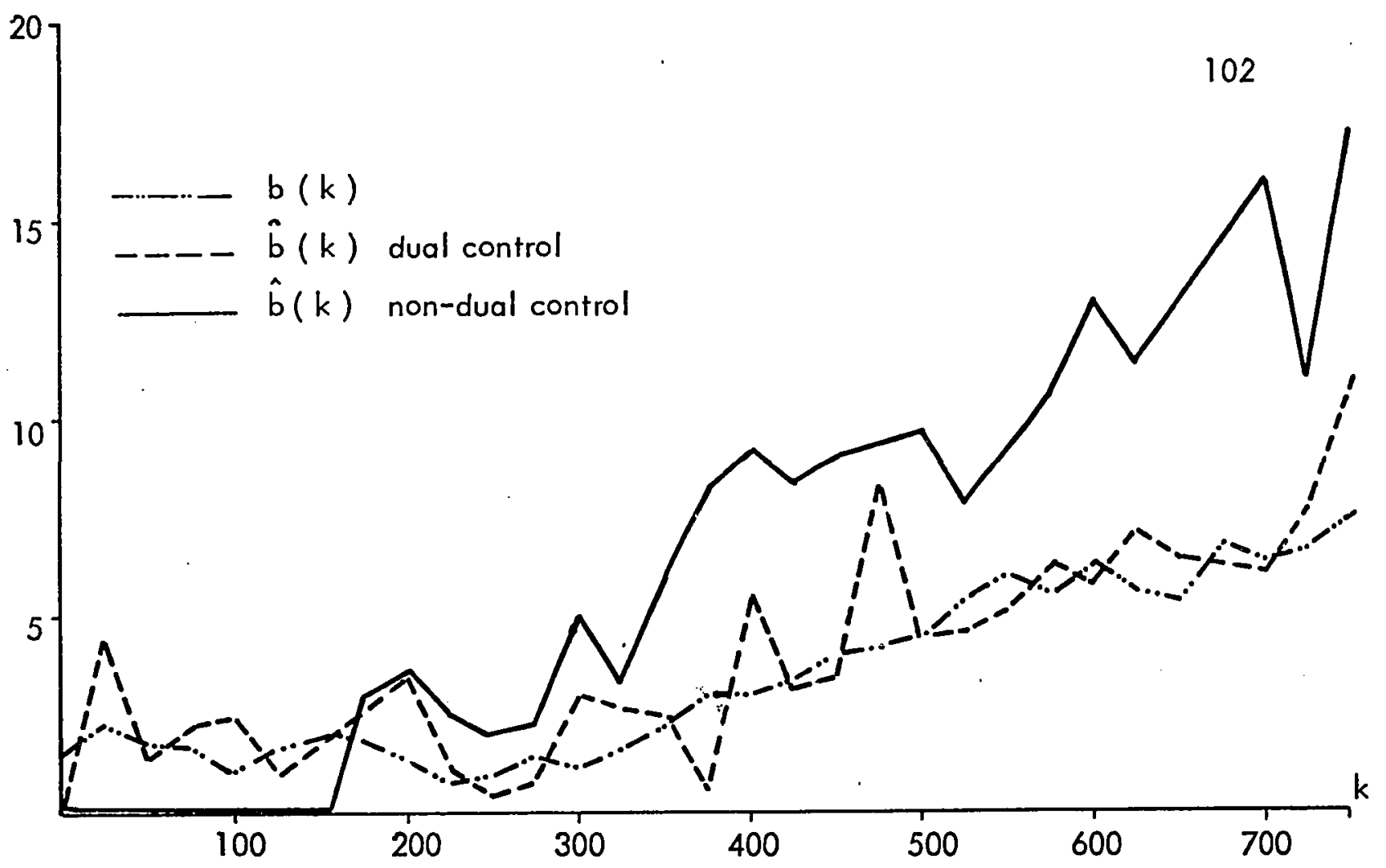

FIGURE 4.12 THE GAIN PARAMETER $b(k)$ AND ITS ESTIMATES, EXAMPLE 4.

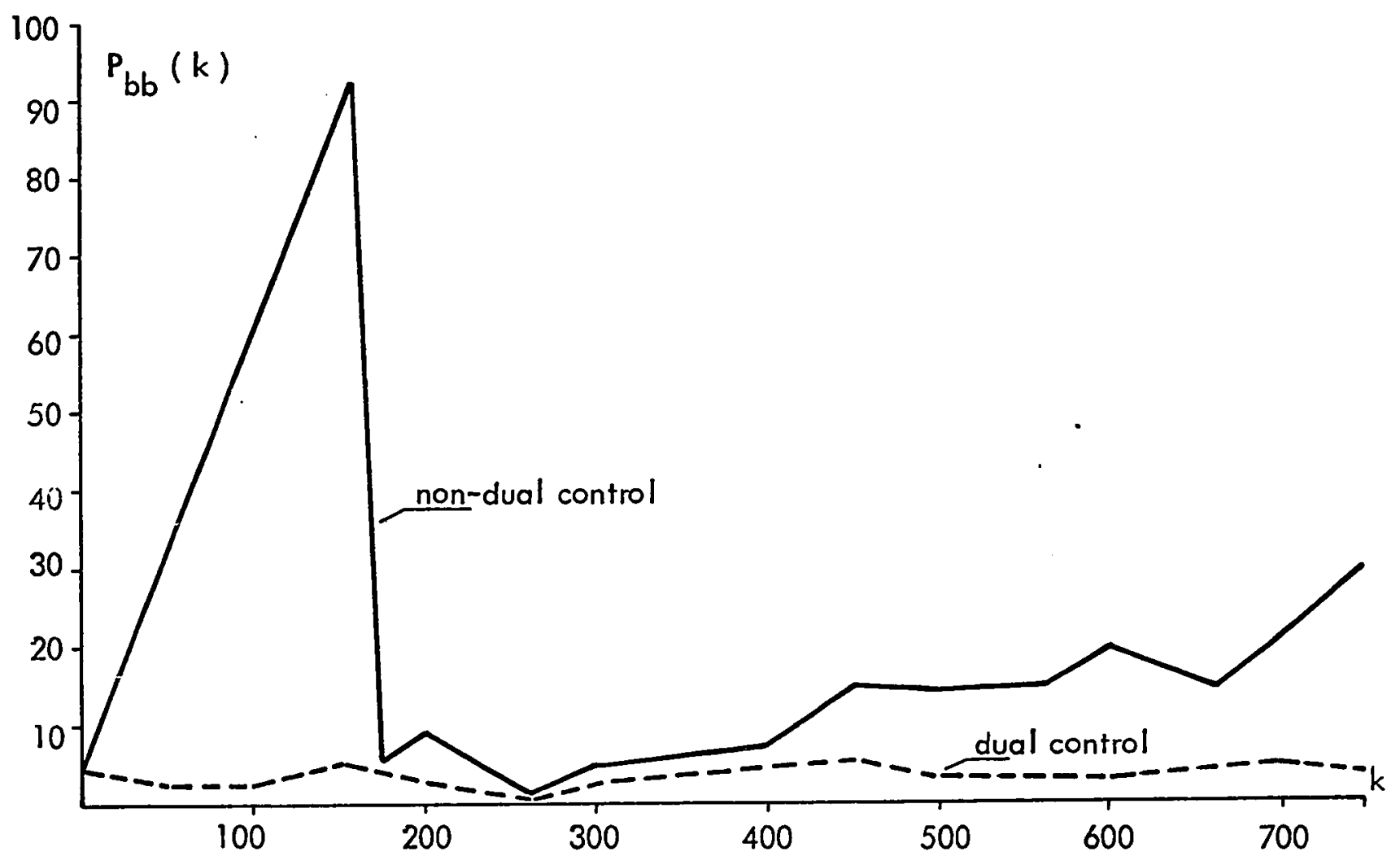

FIGURE 4.13 THE GAIN PARAMETER ERROR VARIANCE, EXAMPLE 4. 
large with a sharp increase in the loss-function as a result.

In Figures 4.12 and 4.13 we see that the error variance of the gain estimate increases constantly while in the turn-off mode, and then decreases sharply at around stage 160, when the turn-off phenomenon terminates, and the identifier starts tracking the gain $b(k)$ once more.

In this example the identification of $b(k)$ as well as of $a(k)$ is constantly poor using the non-dual controller, as compared to the dual controller. This is quite expected since the information about the noise statistics is in error and thus the identification part of the control policy becomes more important.

\subsubsection{Example 5 Repeated Turn-off Phenomena}

Observing the results of Examples 2 and 4 , it may be suspected that the turn-off phenomenon is confined to the initial transient period of the control action. This seems to be the case, since Figures 4.6 and 4.12 indicate the early occurrence of the turn-off (zero gain estimates). To refute this impression we continue in the present example the run used in Example 2, up to the stage 2250. Thus, the results of control simulations are identical to those of Example 2 up to stage 750 , and the curves corresponding to stages 750 to 2250 are given here in Figures $4.14-4.17$. Figure 4.16 shows that using the non-dual control policy, the turn-off phenomenon occurs at stage 1450 approximately, after a long period of good identification. In the same figure we see also that the dual controller ends the turn-off after some time, when the error variance increases to a certain level, 


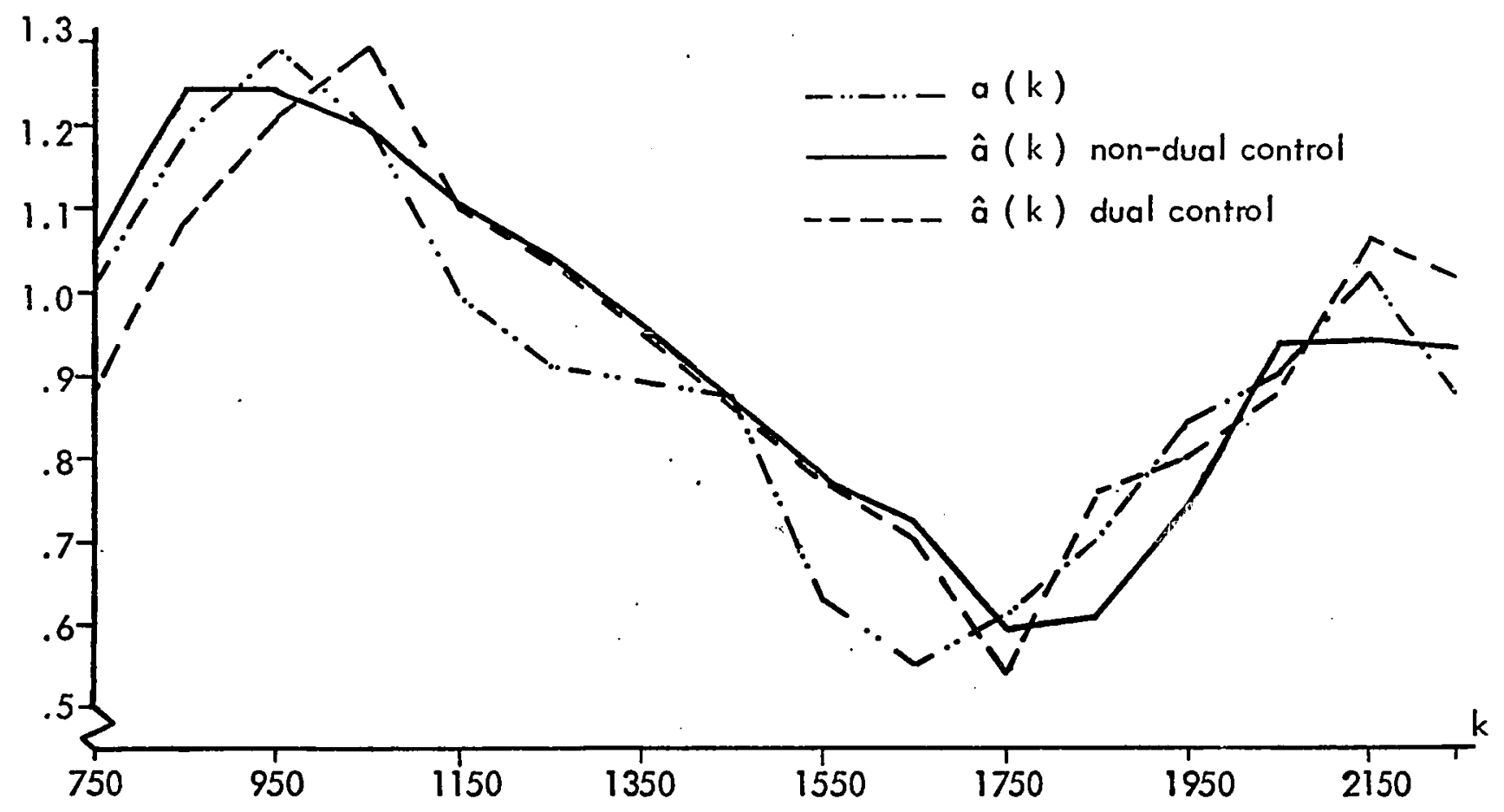

FIGURE 4.14 THE a $(k)$ PARAMETER AND ITS ESTIMATES, EXAMPLE 5.

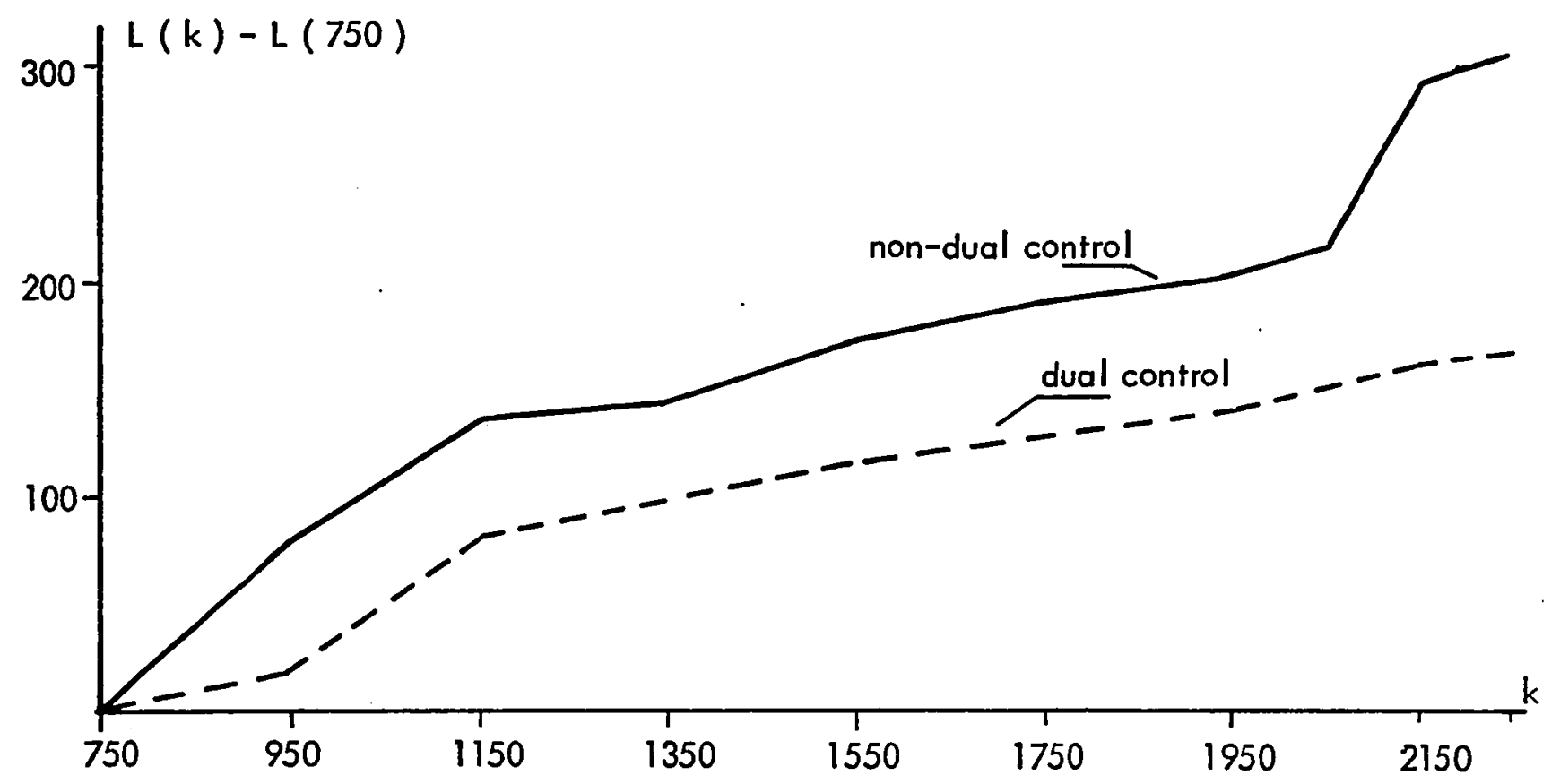

FIGURE 4.15 ACCUMULATED LOSS FUNCTIONS, EXAMPLE 5. 


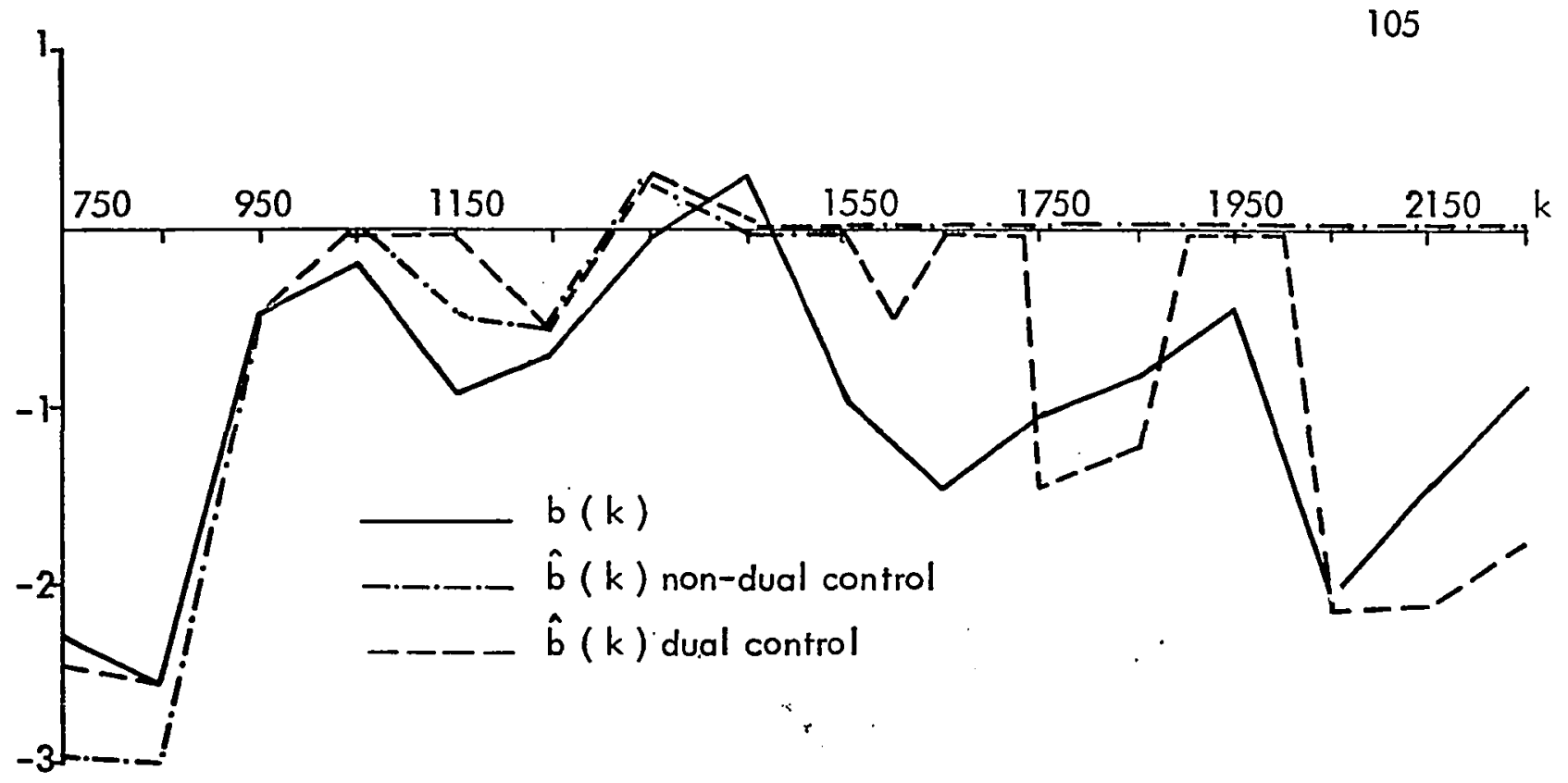

FIGURE 4.16 THE GAIN $b(k)$ AND ITS ESTIMATES, EXAMPLE 5.

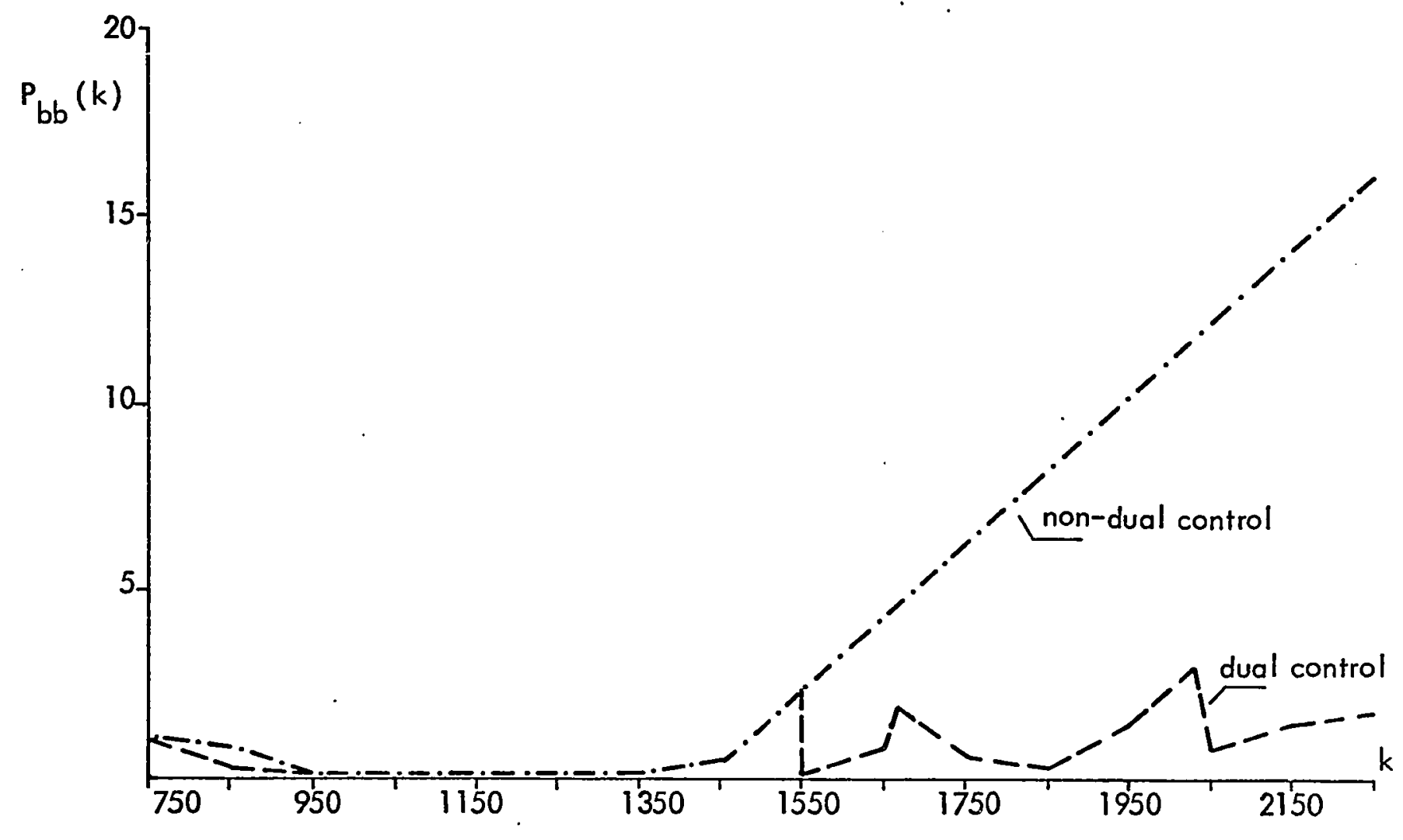

FIGURE 4.17 THE GAIN. $b(k)$ ERROR VARIANCE, EXAMPLE 5. 
as can be seen in Figure 4.17. The estimate $\hat{b}(k+1 \mid k)$ repeatedly becomes almost zero, and the control turned-off. But the dual controller repeatedly terminates this turn-off situations. The loss function is normalized to zero at $k=750$ in Figure 4.15. The steep rise in loss with non-dual control around stage 2100 , corresponds to the combination of turn-off, and parameter $a(k)$ approximately of value 1 .

\subsubsection{Example 6 Two Forms Of Dual Controller}

We now wish to compare the performance of a simple dual controller where the trace constraint is simplest as in (4.23)

$$
\bar{\rho}(k+1)=\rho_{c}
$$

where $\rho_{c}$ is just a constant and an improved dual controller where the trace constraint is given by equation $(4.30)$

$$
\bar{\rho}(k+1)=\left\{\begin{array}{lc}
\rho_{c} & \text { if } P_{b b}(k+1 \mid k) \leq P_{b b}^{M} \\
\frac{a_{b}}{P_{a d}(k+1 \mid k)} & \text { otherwise }
\end{array}\right.
$$

and $\rho_{c}$ is of the form described in Figure 4.1.

The simulation parameters are

$$
Q=\operatorname{diag}\left[\begin{array}{ll}
0.0002 & 0.025
\end{array}\right] ; R=0.04
$$




$$
\begin{aligned}
& x(0) \sim N\left[\gamma_{0}, \Gamma_{0}\right] \\
& \gamma_{0}=\left[\begin{array}{lll}
0.7 & 1.5
\end{array}\right]^{\top} ; \Gamma_{0}=\operatorname{diag}\left[\begin{array}{ll}
0.01 & 0.1
\end{array}\right] \\
& \hat{x}(1 \mid 0)=\left[\begin{array}{ll}
0.1 & 0.1
\end{array}\right]^{\top} \\
& P(1 \mid 0)=\operatorname{diag}\left[\begin{array}{ll}
0.05 & 4.0
\end{array}\right]
\end{aligned}
$$

The accumulated loss $L(k)=\sum^{N} y^{2} \cdot(k)$ and the squared output signal $y^{2}(k)$ $i=1 \quad$ "

were averaged over 50 runs. Figure 4.18 shows the three average lass curves for the non-dual, simple dual, and improved dual control policies. The averaged squared output $\overline{y^{2}}(k)$ was averaged again over each 10 consecutive stages, such that,

$$
\overline{\overline{y^{2}}}(i)=0.1 \sum_{10(i-1)+1}^{10 i} \overline{y^{2}}(k) \quad i=1,2, \ldots, 40
$$

and plotted for the three control cases in Figure 4.19.

The conclusion of this example is clear. Using the simple trace constraint, a constant, yields a large improvement in performance. The use of the improved trace constraint, which monitors the gain parameter error variance $P_{b b}(k)$, results in additional gain in performance.

The dual controller uses the parameters

$$
p_{c}=20, p_{b b}^{M}=2, \quad a_{b}=1.2
$$




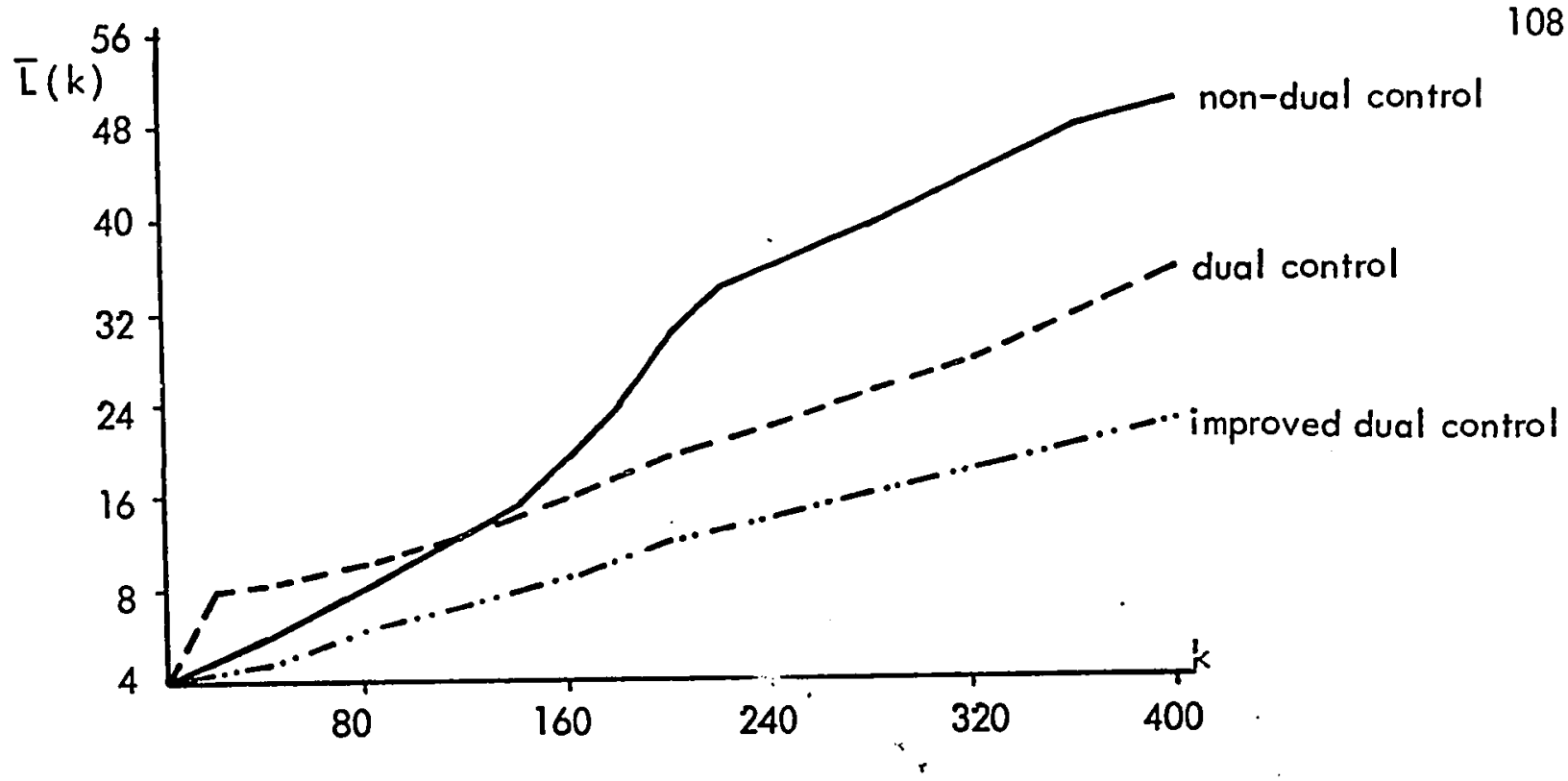

FIGURE 4.18 AVERAGE ACCUMULATED LOSS FUNCTIONS, EXAMPLE 6.

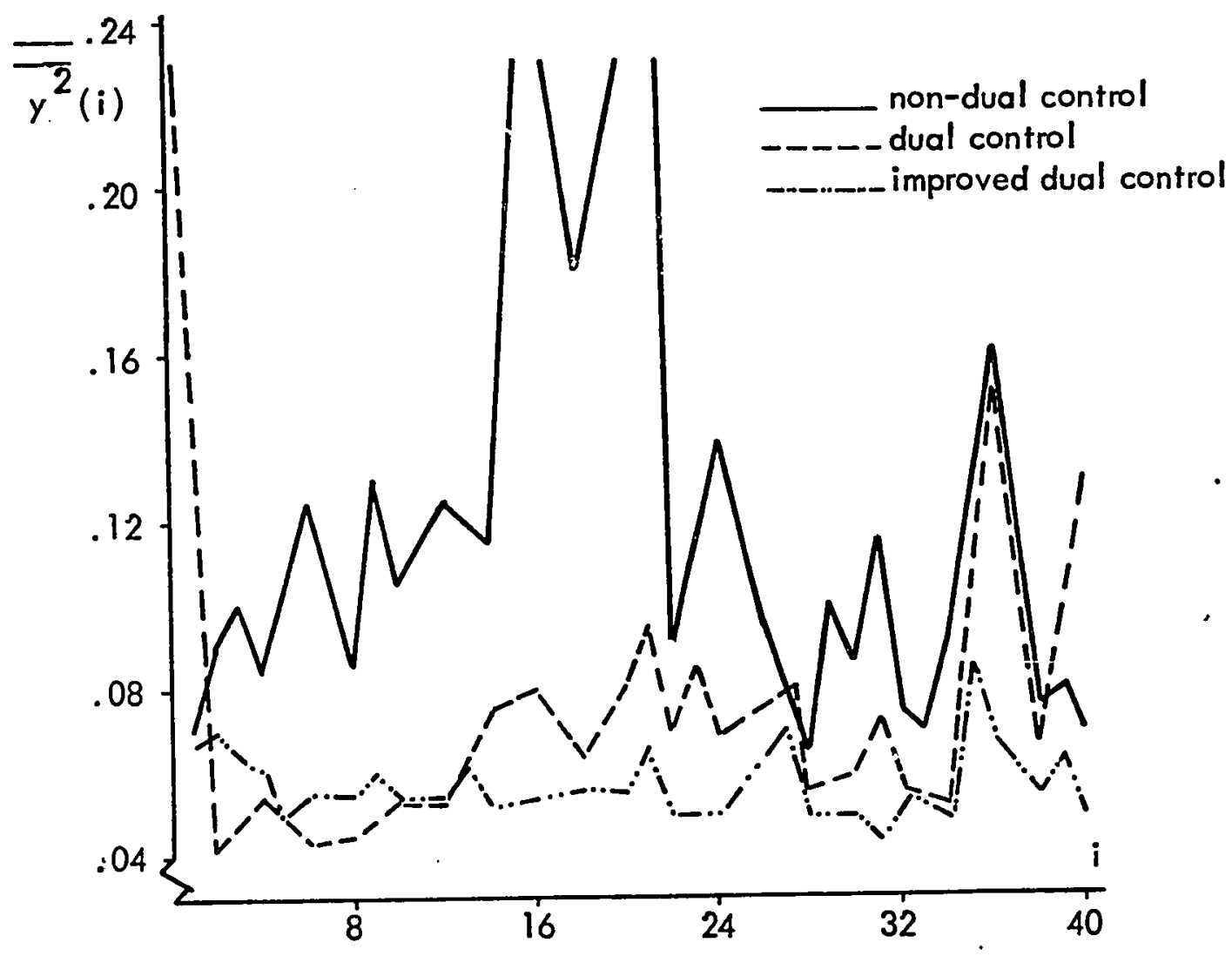

FIGURE 4.19 10-STAGE AVERAGES OF AVERAGE-SQUARED-OUTPUT, EXAMPLE 6. $\quad i=1 k$. 


\subsubsection{Example 7 Steady State Results}

We investiate nov: the adaptive control policies over a long period of time. The plant parameters are simulated here as stationary processes

$$
\begin{aligned}
& a_{1}(k+1)=0.95 a_{1}(k)+\omega_{a}(k) \\
& b_{1}(k+1)=0.95 b_{1}(k)+\omega_{b}(k)
\end{aligned}
$$

with additive constant components

$$
\begin{aligned}
& a(k)=a_{0}+a_{1}(k) \\
& b(k)=b_{0}+b_{1}(k)
\end{aligned}
$$

and have a relatively large driving noise intensity,

$$
Q=\operatorname{diag}\left[\begin{array}{ll}
0.002 & 0.4
\end{array}\right] ; R=0.04
$$

The controller design however, uses the assumed wiener process given by equation (4.4),

$$
\begin{aligned}
& a(k+1)=a(k)+\omega_{a}(k) \\
& b(k+1)=b(k)+\omega_{b}(k)
\end{aligned}
$$

The other parameters of this simulation are

$$
\begin{aligned}
& a_{1}(0) \sim N[0.3,0.1] ; b_{1}(0) \sim N[1 ., 4 .] \\
& a_{0}=0.6 \quad ; b_{0}=2.0
\end{aligned}
$$




$$
\begin{aligned}
& \hat{a}(1 \mid 0)=0.1 ; \quad \hat{b}(1 \mid 0)=0.1 \\
& P(1 \mid 0)=\operatorname{diag}[0.14]
\end{aligned}
$$

The loss curves for the non-dual, dual and perturbation controllers are shown in Figure 4.20, for a sum of 5000 stages.

The performances of the dual and perturbation controllers are equally better than the non-dual controllerras is seen from the difference of the curves slopes.

The parameters here are generated by a stationary process in order to limit their variance at steady state, since the variance of the non-stationary (random walk) model process, grows indefinitely with time.

\subsubsection{Example 8 Steady State Without Exact Knowledge of Noise Covariance}

In this example we compare the performance of the three controllers for the same system as in Example 7, but with the following differences. Here the assumed $Q_{a s}$ is greater than the actual covariance matrix $Q$

$$
Q_{\mathrm{as}}=9 \cdot Q
$$

where

$$
Q=\operatorname{diag}\left[\begin{array}{ll}
0.002 & 0.4
\end{array}\right]
$$

as in the previous example. Also the assumed observation noise variance $R_{\text {as }}$ ' is 


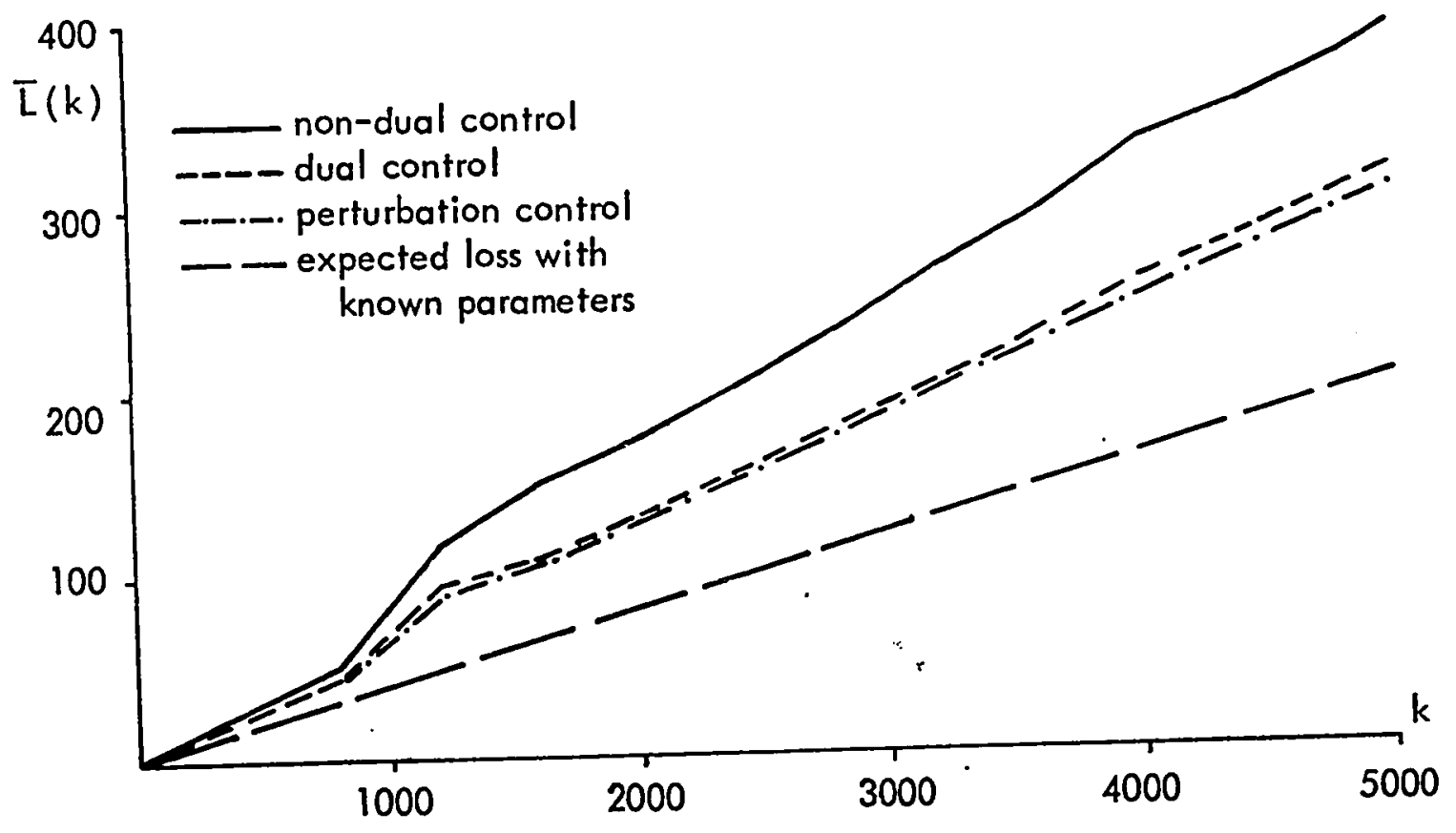

FIGURE 4.20 ACCUMULATED LOSS FUNCTIONS, EXAMPLE 7.

8000

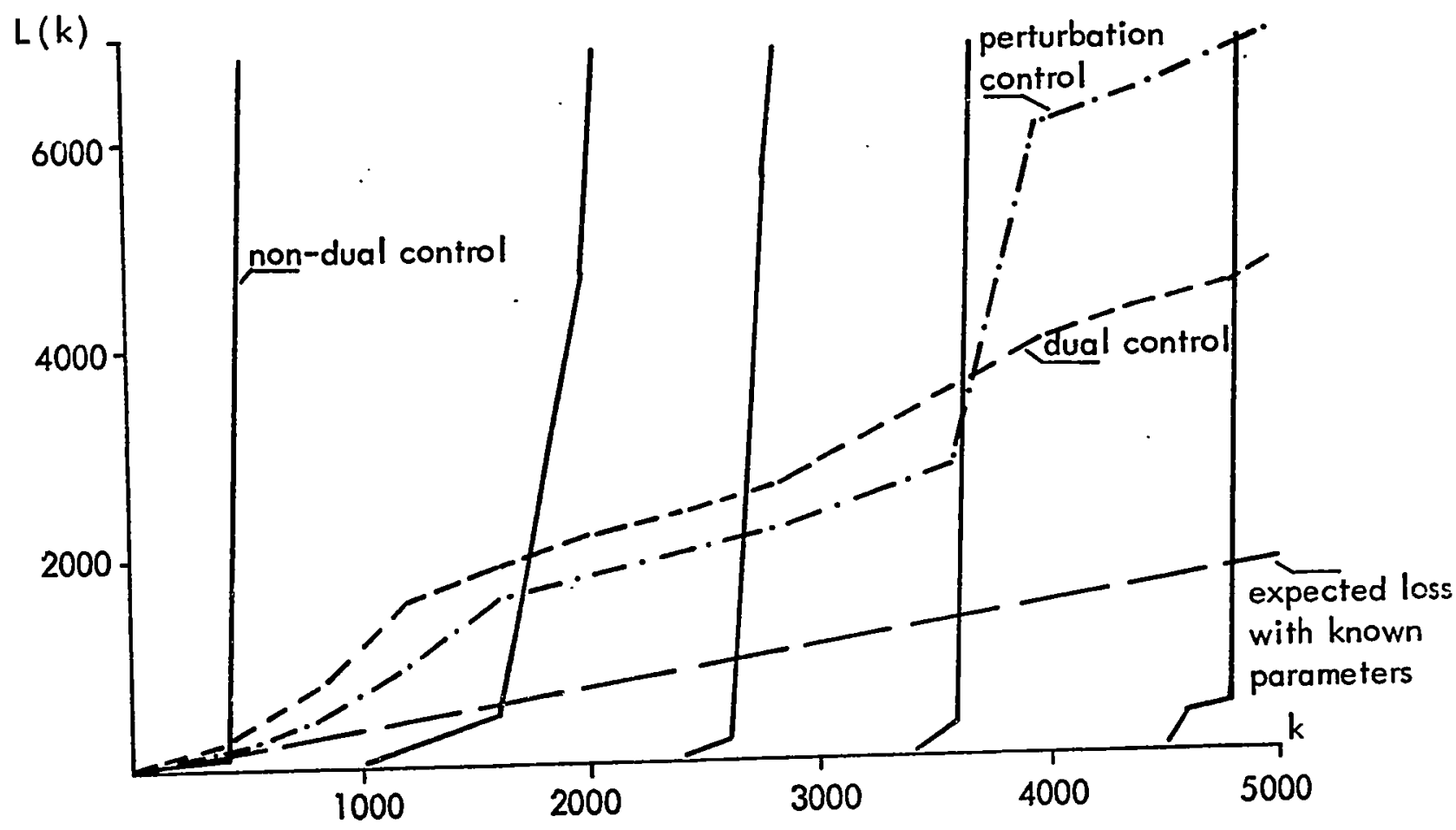

FIGURE 4.21 ACCUMULATED LOSS FUNCTION, EXAMPLE 8. 
smaller than the actual one $R$

$$
R_{a s}=\frac{1}{9} R
$$

where

$$
R=0.36
$$

The assumed noise covariances are used in the control law, with the results shown in Figure 4.21. The identification fails in tracking the parameters, and the burst phenomenon occurs once and again, under the non-dual control law. In order to draw the accumulated loss curve for the non-dual control, which reached a value in the order of $10^{21}$, the accumulated loss $L(k)=\sum_{i=1}^{k} y^{2}(k)$ is reset to zero at the end of each burst. It is significant that the parameter a $(k)$ is strictly less than one and even

$$
a(k) \leq 0.9, \forall k
$$

so that the system is stable along the whole control period. Also the turn-off phenomenon does not occur in this example.

Figure 4.21 demonstrates the great advantage of the dual control law, which by improving the parameters identification, prevents the repeated occurrence of the burst phenomena, thus maintaining satisfactory control.

\subsubsection{Example 9 Deterministic Unknown Parameters}

The present example was used by Wieslander and Wittenmark [4] 
The parameter $a(k)$ and $b(k)$ are deterministic time functions, $a(k)$ is a constant and $b(k)$ is as shown in Figure 4.22. The noise parameter used by the filter are

$$
Q=\operatorname{diag}\left[10^{-7} 2 \cdot 10^{-3}\right] \quad R=0.09
$$

and the initial parameters guess is

$$
\hat{x}(1 \mid 0)=\left[\begin{array}{ll}
0.1 & 0.1
\end{array}\right] .
$$

with initial covariance matrix

$$
P(1 \mid 0)=\left[\begin{array}{ll}
4.0 & 4.0
\end{array}\right]
$$

Figure 4.22 shows that using the non-dual control law the turn-off phenomenon occurs when the gain $b(k)$ decreases from its constant value of 0.5 This turn-off situation remains in this example to the end of the simulation period, with almost zero values for control and $\hat{b}(k)$. Using the dual control technique results in good estimation of $b(k)$ and better control. Figure 4.23 describes the loss curves under non-dual, dual and perturbation control laws, and for no control $u(k)=0$. The turn-off in this case render the system with virtually no control. The identification of the $a(k)$ constant parameter is good, using the dual or non-dual control, and is not shown. The accumulated loss curves of the perturbation and dual controller are virtually identical in this example.

The dual controller parameters are 


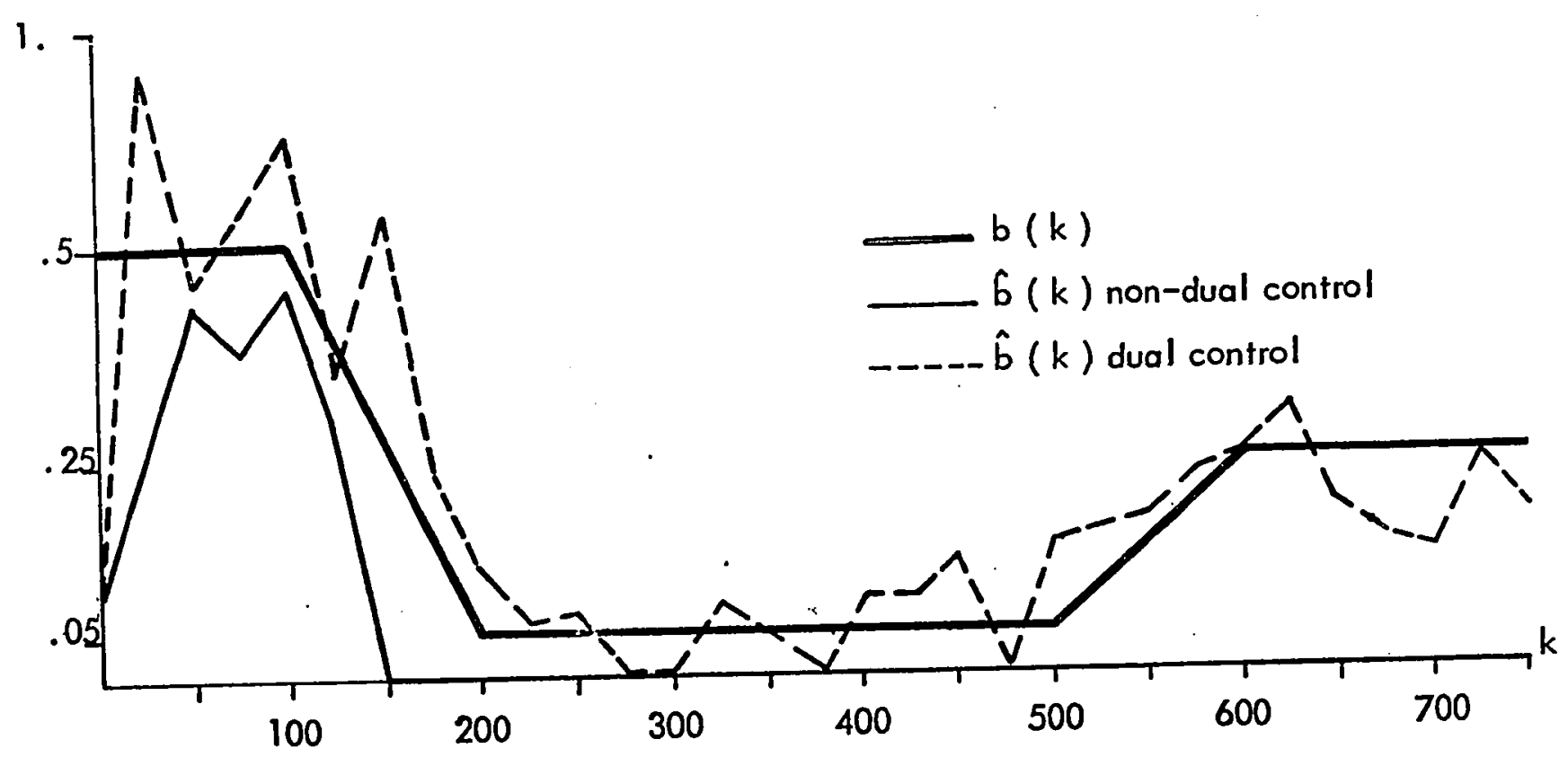

FIGURE 4.22 THE GAIN $b(k)$ AND ITS ESTIMATES, EXAMPLE 9.

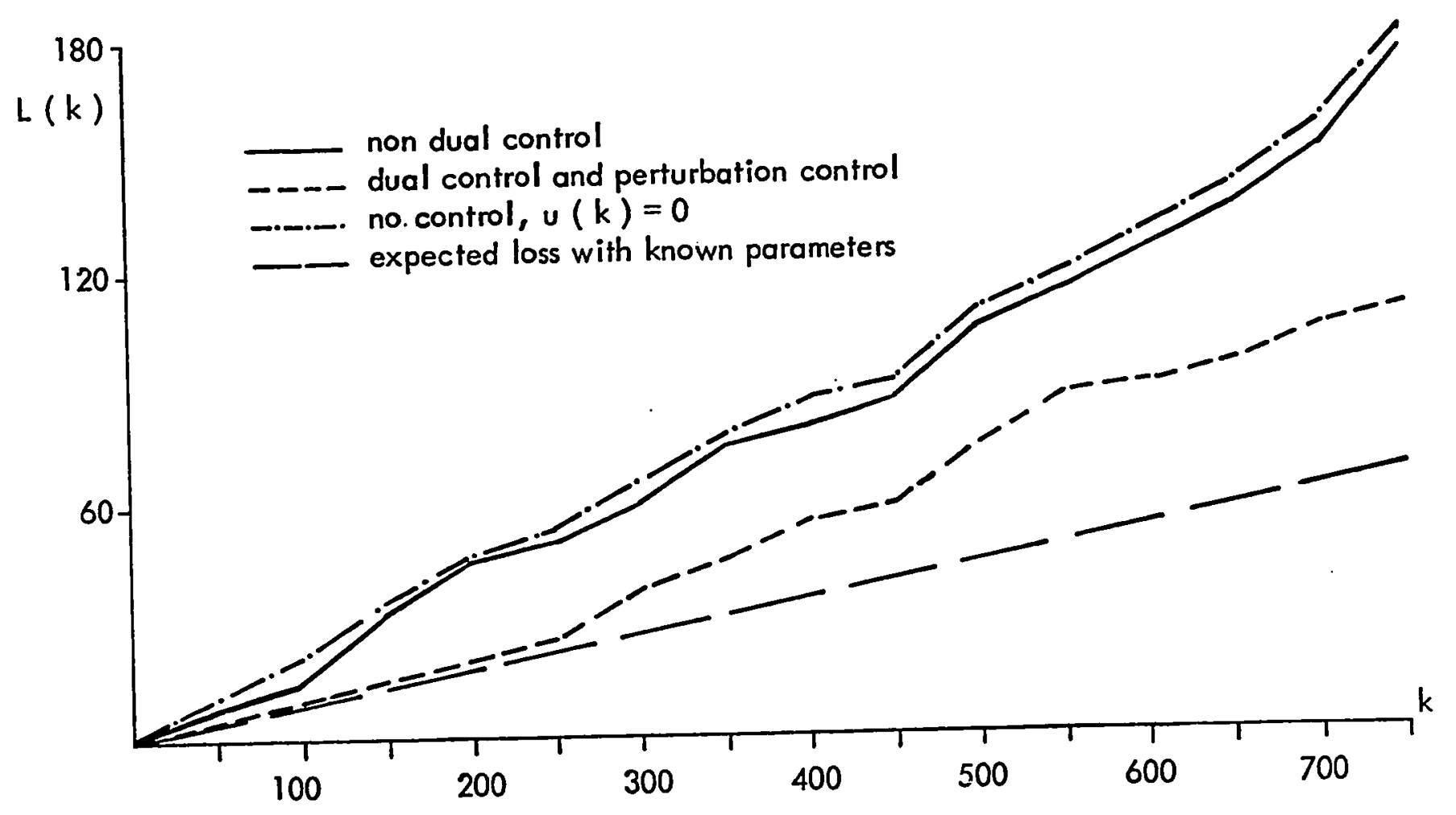

FIGURE 4.23 ACCUMULATED LOSS FUNCTIONS, EXAMPLE 9. 


$$
\rho_{c}=50 ., \mathrm{P}_{\mathrm{bb}}^{M}=4.1, \mathrm{u}^{\mathrm{M}}=0.2 \quad \text { (Equation (4.31)) }
$$

The perturbation controller uses a square wave with amplitude $\delta=0.15$.

\subsubsection{Example 10 Non-Gaussian Parameter Process, Average Performance}

In this example (and in the next one), we depart from the Gaussian process, which we use everywhere else in generating the system random parameters in agreement with our model assumptions.

Consider the event process such that

$$
\text { Prob (event at stage } k \text { ) }=\gamma
$$

with the events being independent, i.e.,

$$
\text { Prob (event at } k \text { event at } i)=\operatorname{Prob}(\text { event at } k), \nabla i \text {. }
$$

This is essential!y $\approx$ discrete Poisson process.

Assume the event to be a discrete impulse, the magnitude of which is Gaussian distributed, with zero mean and variance $\sigma^{2}$. The magnitudes of impulses at different times are independent. This process is white and has a variance of $\gamma \sigma^{2}$.

Let $\omega(k)$ be that process, and let

$$
\begin{aligned}
& a(k+1)=a(k)+\omega_{a}(k) \\
& b(k+1)=b(k)+\omega_{b}(k)
\end{aligned}
$$


where

$$
\begin{aligned}
& \omega(k)=\left[\omega_{a}(k) \quad \omega_{b}(k)\right]^{\top} \\
& \operatorname{cov}\left[\omega(k) ; \omega(i)=\operatorname{diag}\left[\gamma \sigma_{a}^{2} \quad \gamma \sigma_{b}^{2}\right] u_{o}(k \cdot i)\right.
\end{aligned}
$$

Since our controller depends only on second order statistics, the Kalman filter that is used, is the optimal linear filter for this process.

The system to be controlled is simulated using the above process for the parameters. Fifty runs are simulated, and the average accumulated loss functions are computed and shown in Figure 4.24, for the dual, non-dual and perturbation controllers.

The non-dual controller experiences many turn-offs, which are prevented by the dual and perturbation controllers.

The parameters noise process is simulated with

$$
\sigma_{a}^{2}=0.001 ; \quad \sigma_{b}^{2}=0.1
$$

and the event probability is

$$
\gamma=0.05
$$

The actual covariance of the process is thus

$$
Q=\operatorname{diag}\left[\begin{array}{ll}
0.001 & 0.1
\end{array}\right] \cdot 0.05
$$

but the filter uses the assumed covariance 


$$
Q_{a s}=\operatorname{diag}\left[\begin{array}{ll}
0.01 & 0.1
\end{array}\right]
$$

The observation noise is $v(k) \sim N[0, R], R=0.04$.

\subsubsection{Example 11 Non-Gaussian Parameter Process, Single Run Results}

Here we give some results of one run of the 50 , used in the previous example. The dual and non-dual controllers are compared in Figure 4.25, which describes their respective accumulated loss functions. Figures 4.26 and 4.27 show the $b(k)$ and $a(k)$ parameters respectively, as well as their respective estimates under the two control laws.

Figure 4.26 shows the turn-off phenomenon occuring while using the non-dual controller at stage $220, \hat{b}(k)$ is virtually equal to zero. The identification of the gain parameter is much better under the dual policy.

The identification of $a(k)$ is good with both controllers, as is seen in Figure 4.27. The noise intensity in the generation of $a(k)$ was taken relatively small, as otherwise the system becomes unstable with the non-dual controller, because of the frequent turn-off phenomena.

\subsection{Second Order System Simulation}

In this section we apply the dual control policy to a system of second order with three random parameters. The model representation in this case is 


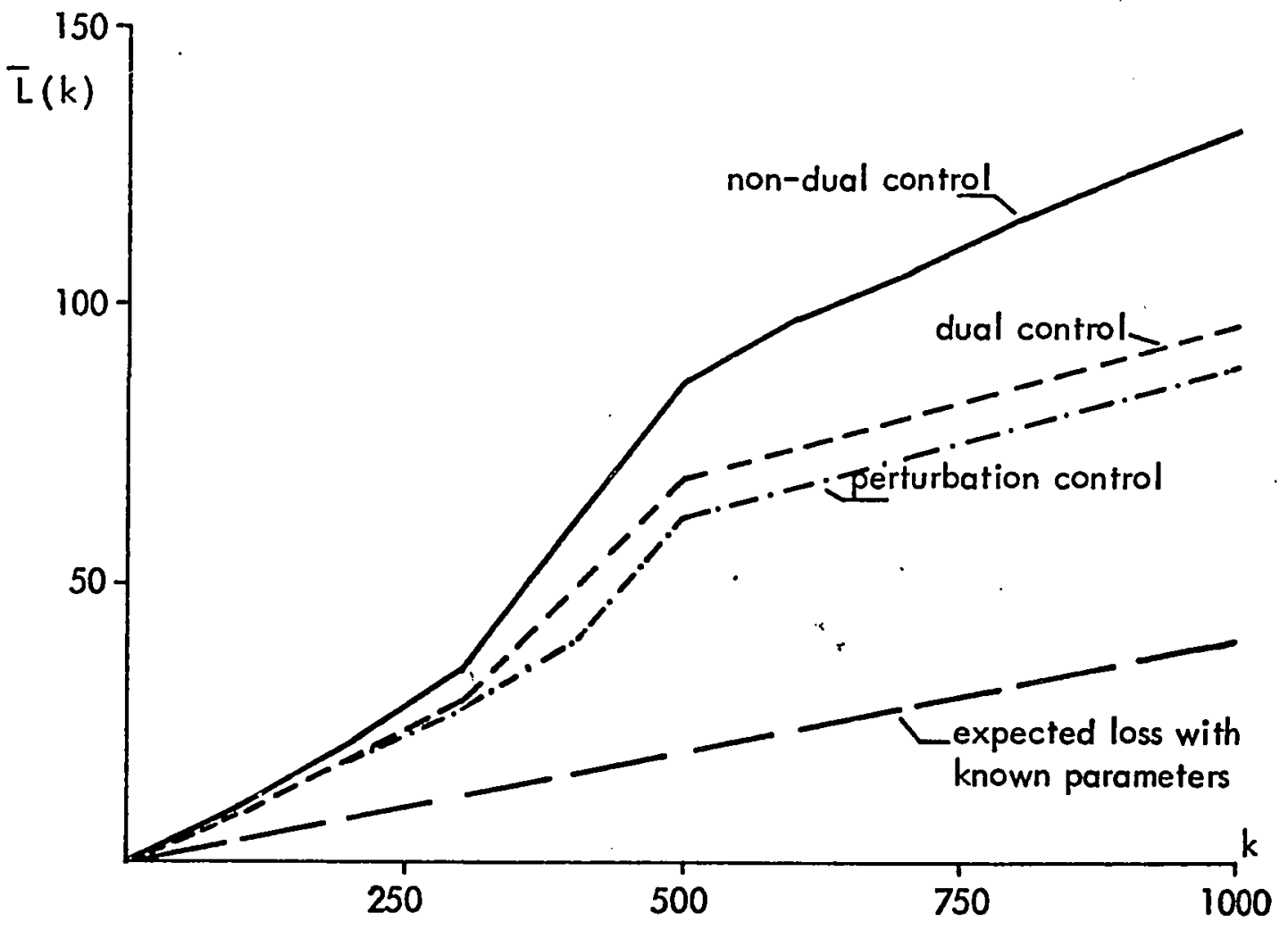

FIGURE 4.24 AVERAGE ACCUMULATED LOSS FUNCTIONS, EXAMPLE 10.

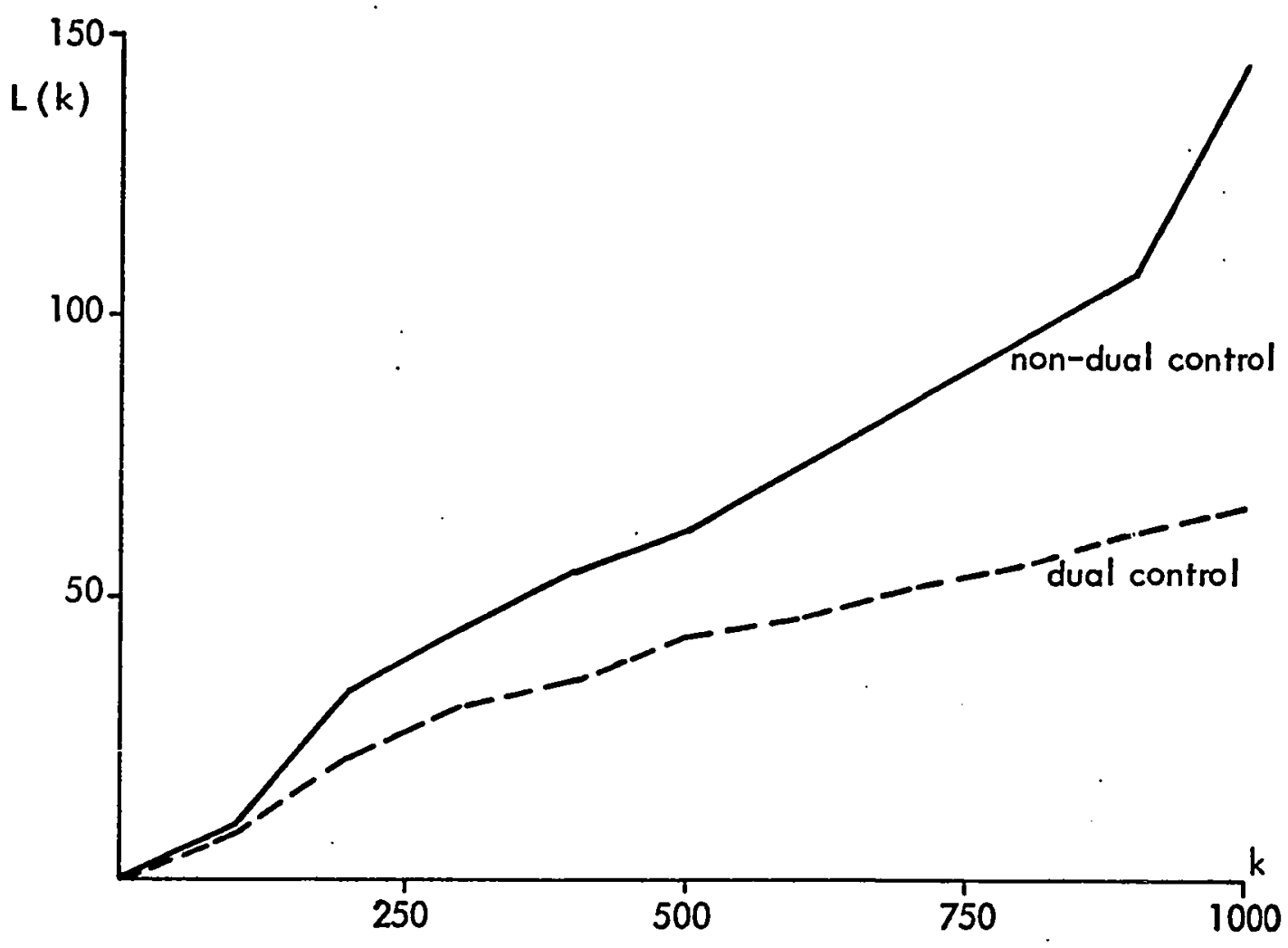

FIGURE 4.25 ACCUMULATED LOSS-FUNCTIONS, SINGLE RUN, EXAMPLE 11. 


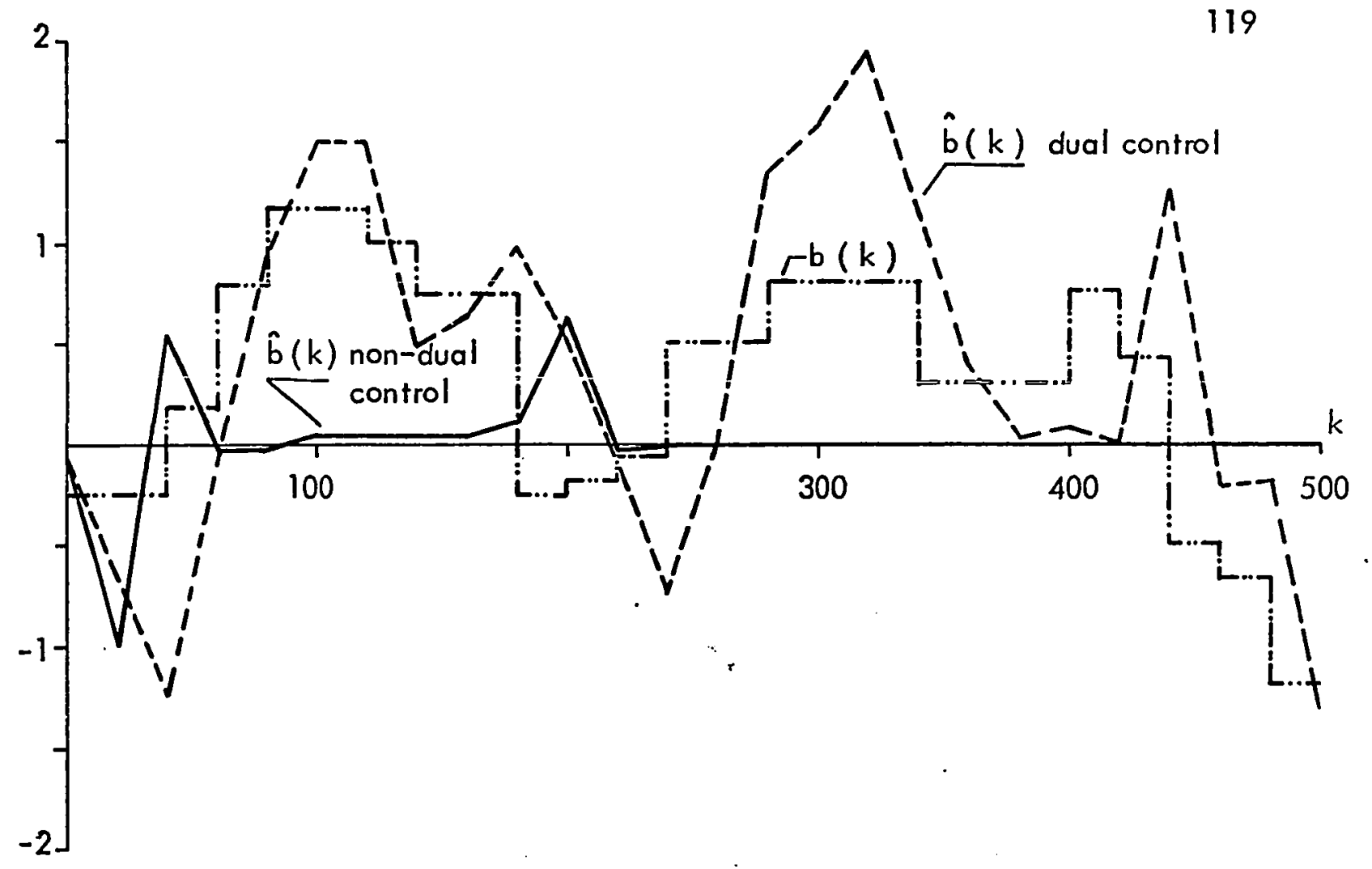

FIGURE 4.26 THE $b(k)$ PARAMETER AND ITS ESTIMATES, EXAMPLE 11.

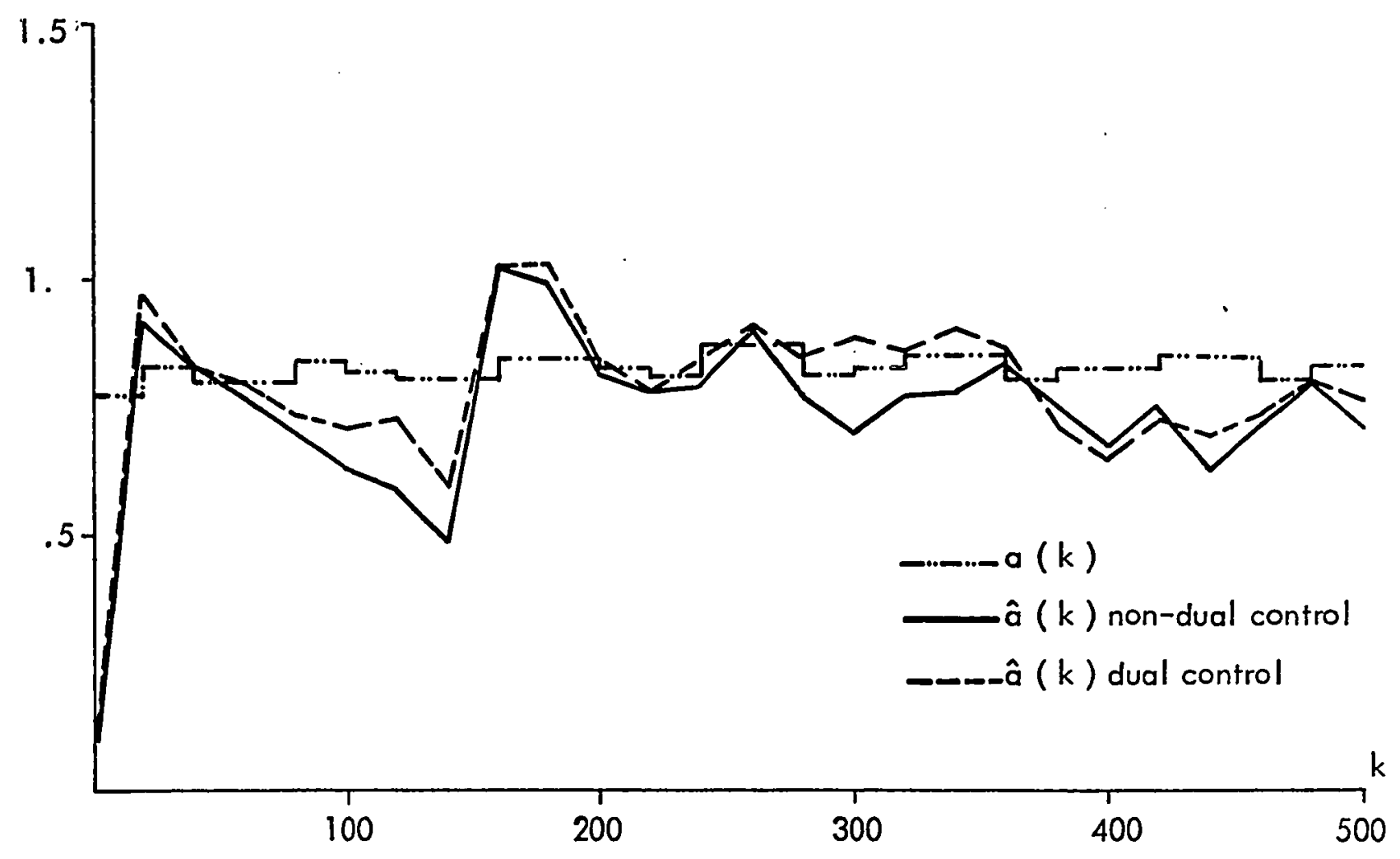

FIGURE 4.27 THE a $(k)$ PARAMETER AND ITS ESTIMATES, EXAMPLE 11. 


$$
y(k)=a_{1}(k) y(k-1)+a_{2}(k) y(k-2)+b(k) u(k-1)+v(k)
$$

which can be written in the form of (3.5), (3.7)

$$
\begin{aligned}
& y(k)=\varphi(k-1) x(k)+v(k) \\
& x(k+1)=x(k)+\omega(k)
\end{aligned}
$$

where

$$
\begin{aligned}
& \varphi(k-1)=[y(k-1) y(k-2) u(k-1)] \\
& x(k)=\left[\begin{array}{lll}
a_{2}(k) & a_{2}(k) b(k)
\end{array}\right]^{\top} \\
& \omega(k)=\left[\begin{array}{ll}
\omega_{a_{1}}(k) \omega_{a_{2}}(k) \omega_{b}(k) & ]^{\top} \sim N[0, Q]
\end{array}\right.
\end{aligned}
$$

and

$$
\begin{aligned}
& Q=\operatorname{diag}\left[\begin{array}{lll}
q_{a_{1}} & q_{a_{2}} & q_{b}
\end{array}\right] \\
& v(k) \sim N[0, R]
\end{aligned}
$$

The control Equation (3.22) is in the case

$$
u^{0}(k)=-\frac{\hat{b}(k+1 \mid k) \hat{a}_{c}^{\top}(k+1 \mid k)+P_{3}^{\top}(k+1 \mid k)}{\hat{b}^{2}(k+1 \mid k)+P_{b b}(k+1 \mid k)} Y(k)
$$

where 


$$
\begin{aligned}
& \hat{a}_{c}^{\top}(k+1 \mid k)=\left[\begin{array}{ll}
\hat{a}_{1}(k+1 \mid k) & \hat{a}_{2}(k+1 \mid k)
\end{array}\right] \\
& P_{3}^{\top}(k+1 \mid k)=\left[\begin{array}{lll}
P_{a_{1} b}(k+1 \mid k) & P_{a_{2} b}(k+1 \mid k)
\end{array}\right] \\
& Y(k)=[y(k) \quad y(k-1)]^{\top}
\end{aligned}
$$

The dual, non-dual and perturbation controllers are applied to this second order system, and the respective performances are compared. The controllers and filter used, are described in Section 4.2.

Next we give two examples. The first is concerned a comparison of average performances of the different controllers, and the second one investigates the identification and control results of a single run.

\subsubsection{Example 1 Comparison of Average Performance}

The average accumulated loss functions are computed in this example using a 50 runs system simulation, with random noise samples, and random initial conditions. Each run is simulated with the dual, non-dual and perturbation controllers, and the resulting average loss curves are shown in Figure 4.28.

The simulation parameters are as follows:

$$
\begin{aligned}
& Q=\operatorname{diag}\left[\begin{array}{lll}
2.10^{-5} & 2.10^{-5} & 6.10^{-3}
\end{array}\right] \\
& R=0.04 \\
& x(1) \sim N\left[\gamma_{0}, \Gamma_{0}\right] \\
& \gamma_{0}=\left[\begin{array}{lll}
0.3 & 0.3 & 1 .
\end{array}\right]^{\top}
\end{aligned}
$$




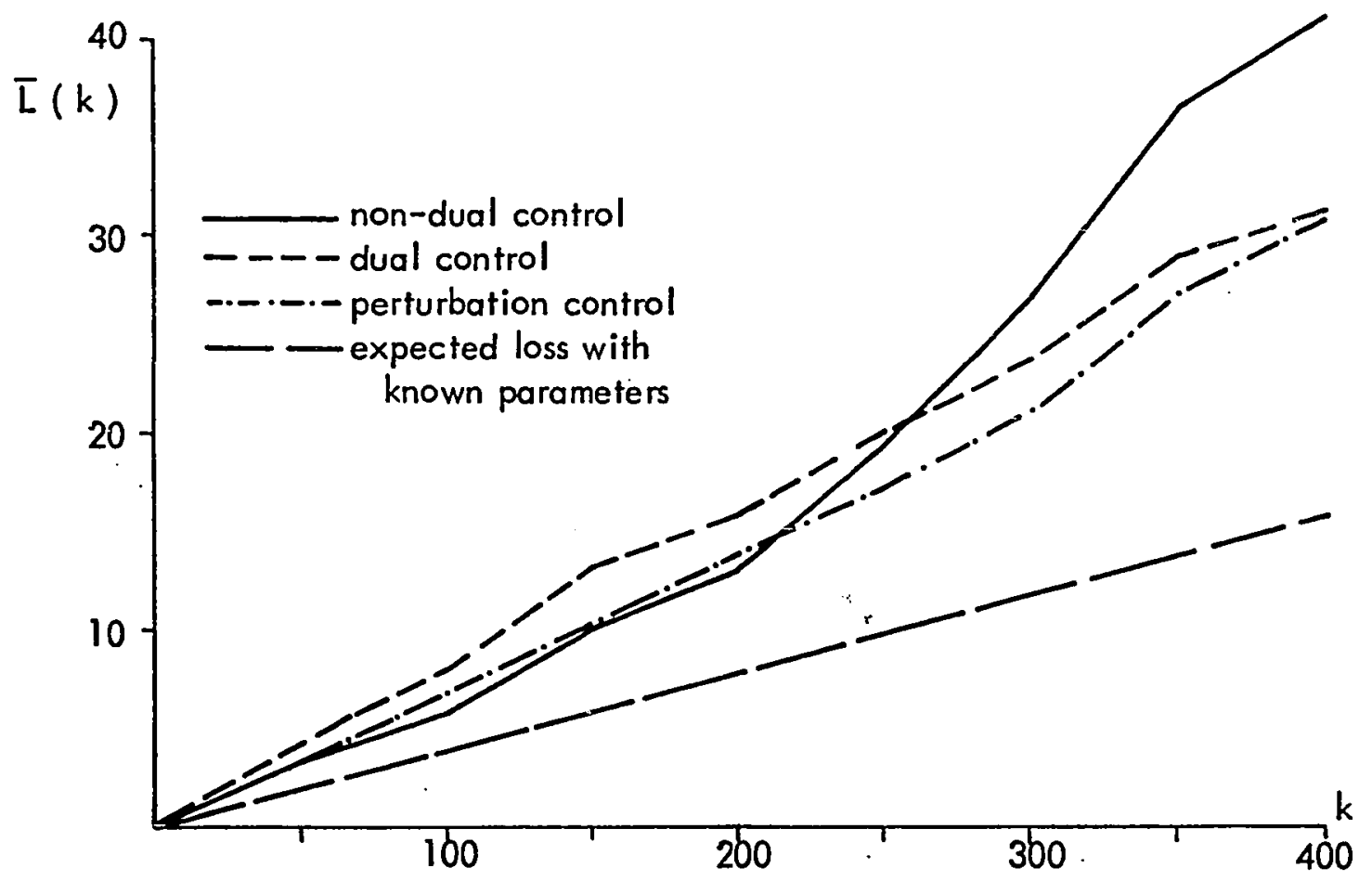

FIGURE 4.28 AVERAGE ACCUMULATED LOSS FUNCTIONS, EXAMPLE 1.

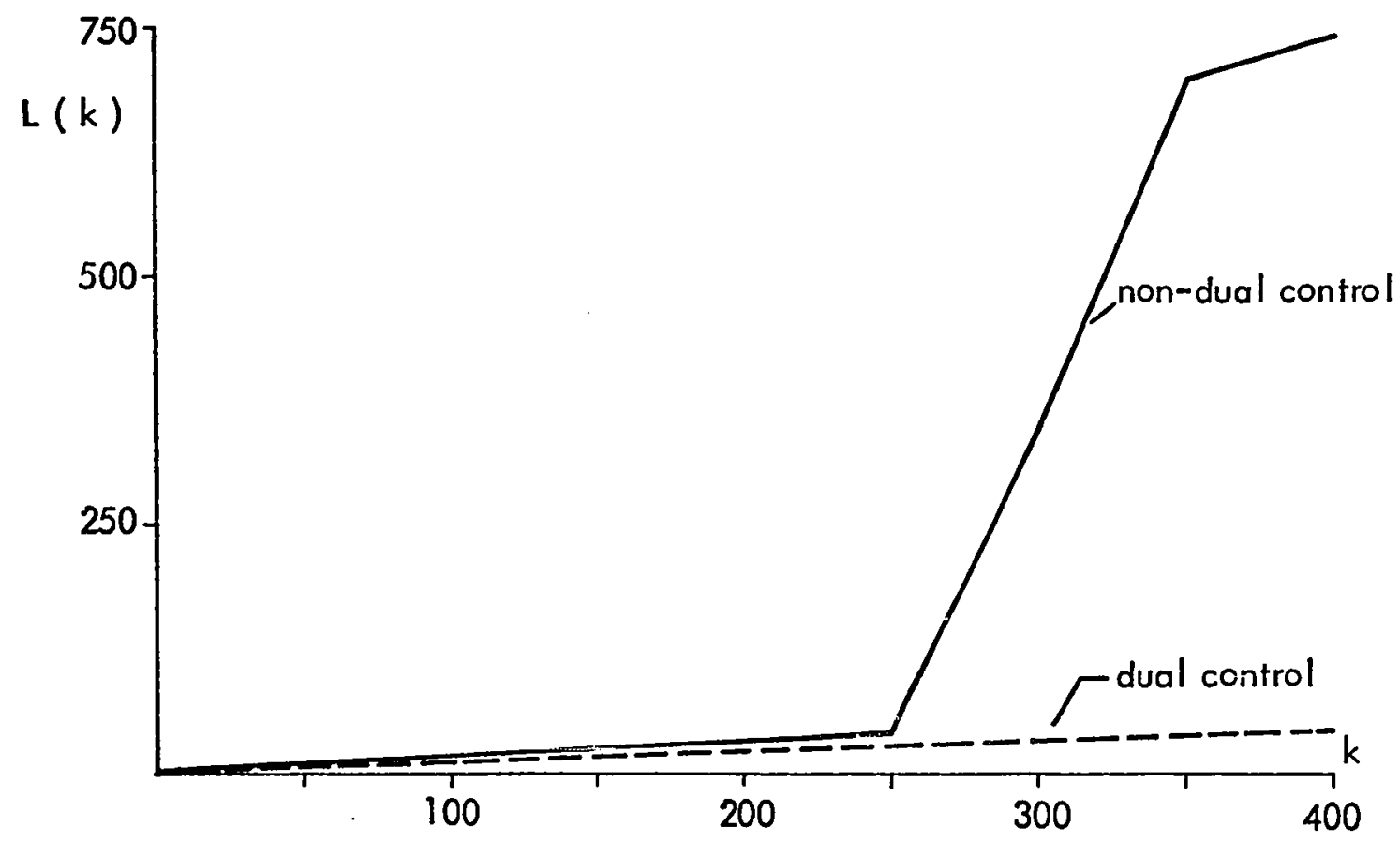

FIGURE 4.29 ACCUMULATED LOSS FUNCTIONS, EXAMPLE 2. 


$$
\begin{aligned}
& \Gamma_{0}=\operatorname{diag}\left[\begin{array}{lll}
0.1 & 0.1 & 4 .
\end{array}\right] \\
& \hat{x}(1 \mid 0)=\left[\begin{array}{lll}
0.1 & 0.1 & 0.1
\end{array}\right]^{\top} \\
& P(1 \mid 0)=\left[\begin{array}{lll}
4 . & 4 . & 4 .
\end{array}\right]
\end{aligned}
$$

The dual controller uses, in this simulation, a trace constraint

of a simple form

$$
\rho(k+1)=\rho_{C}
$$

where $\rho_{c}$ is piecewise constant as follows:

$$
\begin{array}{ll}
\rho_{c}=0 . & k \leq 15 \\
\rho_{c}=40 . & 15<k<100 \\
\rho_{c}=250 . & 100 \leq k<300 \\
\rho_{c}=450 . & , \quad 300 \leq k
\end{array}
$$

The perturbation controller uses a square wave perturbation signal, of amplitude $\delta=0.01$.

Figure 4.28 demonstrates the advantage of the dual control policy over the non-dual one. In this case the perturbation controller performance, is almost equal and somewhat better than that of the dual one.

\subsubsection{Example 2 Single Run Results}

We sho: in this example some of the identification results, of one of 


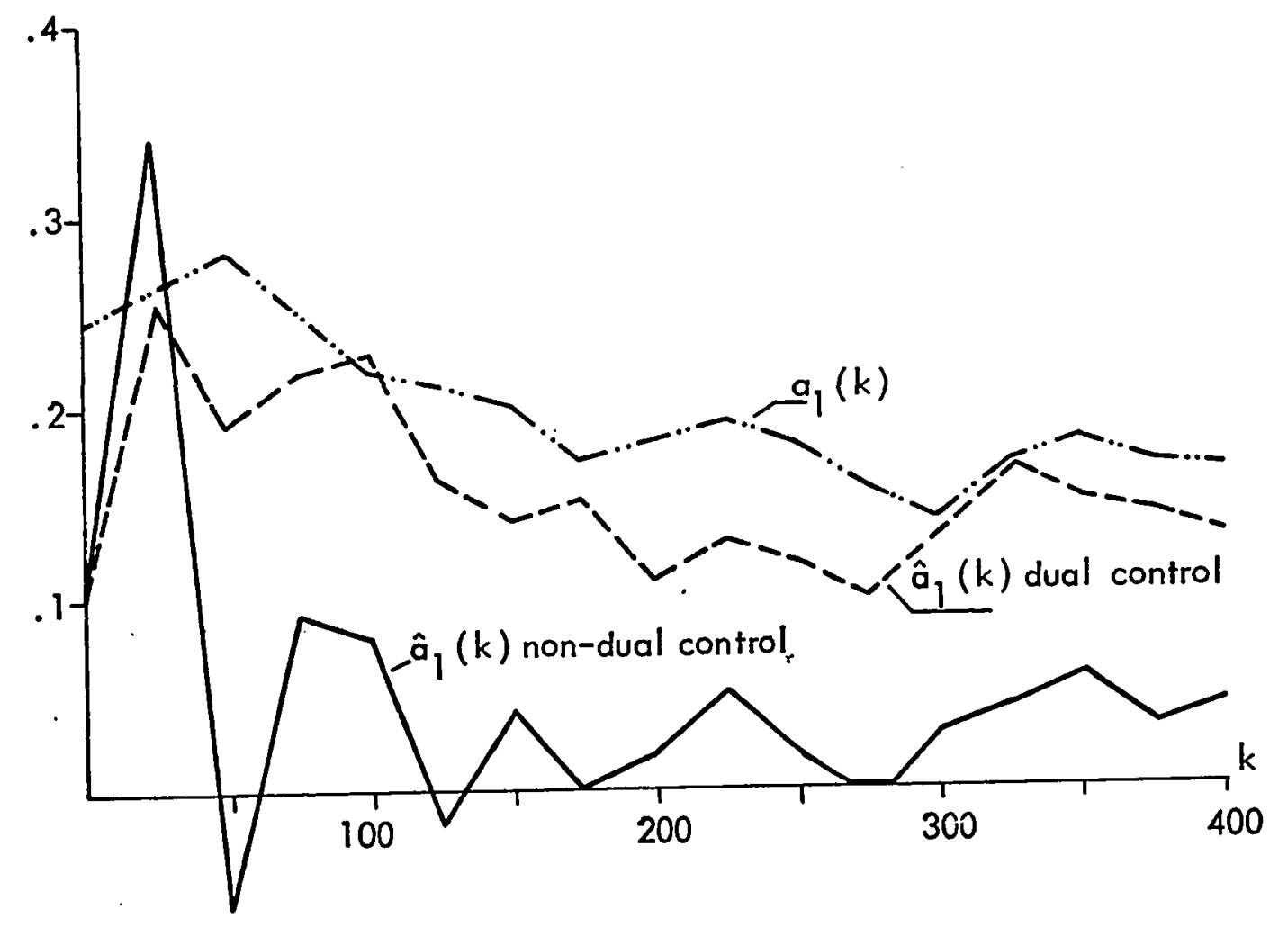

FIGURE 4.30 THE $a_{1}(k)$ PARAMETER AND ITS ESTIMATES, EXAMPLE 2.

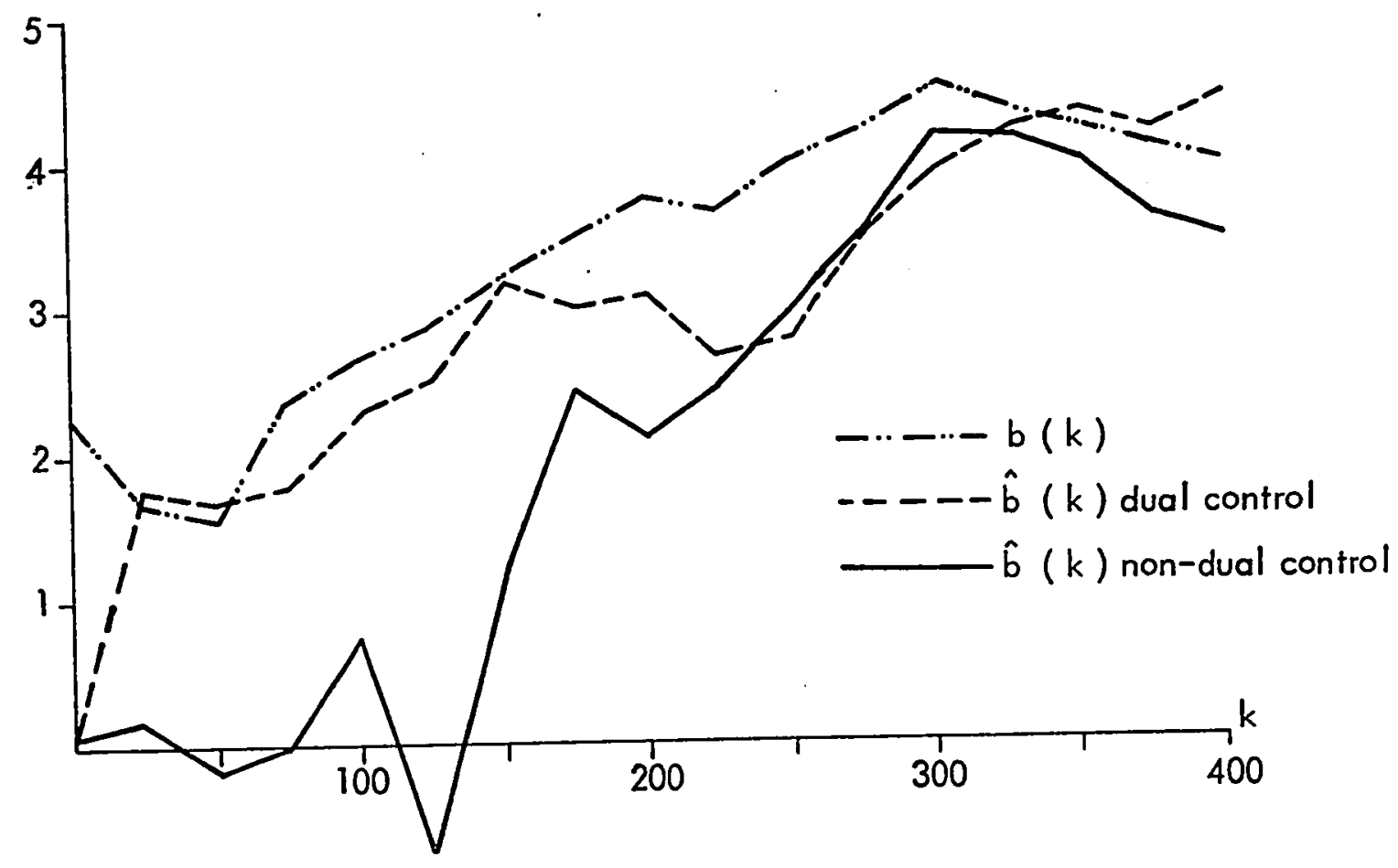

FIGURE 4.31 THE GAIN PARAMETER $b(k)$ AND ITS ESTIMATES, EXAMPLE 2. 
the fifty runs that were used in the previous example. In this run the dual and nondual controller perform equally well up to about stage 250 , as can be seen from their respective loss curves in Figure 4.29. This is true in spite of the fact that the identification of the parameters $a_{1}(k)$ and $b(k)$, is quite poor under the nondual policy. However the non-dual controller is adaptive, in the sense that it depends not only on the parameter estimates but also on the estimation error variance. This dependence or the estimation error serves. well up to stage 250 , but is not capable in preventing a deterioration in control, in the interval $[250,350]$, which is then followed by a period of good control due to better identification. Figures 4.30 and 4.31 describe the parameters $a_{1}(k), b(k)$ and their estimates $\hat{a}_{1}(k)$ and $\hat{b}(k)$. The superiority of the dual controller is clearly demonstrated. The estimate of the parameter $a_{2}(k)$ is not shown as its identification is good with both controllers. 


\section{CHAPTER V}

\section{ADAPTIVE CONTROL WITH ADAPTIVE FILTERING}

\subsection{Introduction}

In a recent paper [9] P.R. Bélanger derives an algorithm for the estimation of noise covariance matriçes for linear, discrete, time-varying stochastic processes. We apply in this chapter his results to construct an adaptive filter. The noise variances are unknown and must be estimated. By using Bélanger's algorithm and treating the covariance matrices as time varying parameters we update them through real-time identification and use the updates in the filter, thus obtaining adaptive filtering. This adaptive filter together with the dual adaptive control policy yields an overall adaptive scheme for stochastic systems with uncertain noise parameters.

\subsection{Development Of The Adaptive Filter}

We assume that the noise variances $R$ and $Q$ are not known. The Kalman filter used by the adaptive control policy is thus not optimal. Applying the method of Bélanger [9], we estimate on line, at each stage, the noise variances, and use the updated values in the filter equations, thus obtaining an adaptive filter that uses progressively better noise variances estimates.

To derive the adaptive filter we use notations similar to those used by Bélanger. The system given in equations (3.5) and (3.7) is now written as, 


$$
\begin{aligned}
& x(k)=A(k-1) x(k-1)+\omega(k-1) \\
& y(k)=H(k) x(k)+v(k)
\end{aligned}
$$

where the matrix $A(k-1)$ is included for generality, and

$$
H(k) \triangleq \varphi(k-1)
$$

$x(k)$ and $\varphi(k-1)$ are as in Equations. (3.4) and (3.6) respectively, and

$$
\begin{aligned}
& \omega(k) \sim N[0, Q] \\
& v(k) \sim N[0, R]
\end{aligned}
$$

where $R$ and $Q$ are constant unknown scelar and matrix respectively.

$$
\text { Assume that } R \text { and } Q \text { can be represented in the form of a }
$$

linear combination,

$$
\begin{aligned}
& R=\sum_{i=1}^{N} a_{i} R_{i} \\
& Q=\sum_{i=1}^{N} a_{i} Q_{i}
\end{aligned}
$$

where $R_{i}$ and $Q_{i}$ are known. The problem is to find $\hat{\alpha}$ - the best estimate of the vector $\alpha$

$$
a=\left[\alpha_{1} \alpha_{2} \ldots a_{N}\right]^{\top}
$$


Assume a filter of the form

$$
\begin{aligned}
& \hat{x}(k \mid k-1)=A(k-1) \hat{x}(k-1 \mid k-2)+K(k-1) \nu(k-1) \\
& \nu(k)=y(k)-H(k) \hat{x}(k \mid k-1)
\end{aligned}
$$

where $\nu(k)$ is the innovation sequence $[10]$, and where the gain sequence $K(k)$ is to be chosen such that, the filter is uniformly asymptotically stable. We choose the gain as

$K(k)=A(k) P(k \mid k-1) H^{\top}(k)\left[H(k) P(k \mid k-1) H^{\top}(k)+\hat{R}(k)\right]^{-1}$

where we use for the unknown covariance $R$, its estimate at stage $k$, such that

$$
\hat{R}(k)=\sum_{i=1}^{N} \hat{a}_{i}(k) R_{i}
$$

$\hat{\alpha}_{i}(k)$ being the estimate of $\alpha_{i}$ at stage $k$. The covariance matrix sequence satisfies

$P(k \mid k-1)=\Phi(k-1) P(k-1 \mid k-2) \Phi^{\top}(k-1)+\hat{Q}(k)+K(k) \hat{R}(k) K^{\top}(k)$

where again

$$
\hat{Q}(k)=\sum_{i-1}^{N} \hat{a}_{i}(k) Q_{i}
$$

is the estimate of $Q$ at stage $k$, and 


$$
\Phi(k)=A(k)-K(k) H(k)
$$

If $R$ and $Q$ were known, the gain sequence $K(k)$, using these covariances, will be optimal and the innovation sequence $\nu(k)$ will be white. We use the Kalman gain (5.8) with the estimates $\hat{R}(k)$ and $\hat{Q}(k)$. Thus the gain is not optimal but the resulting filter is uniformly asymptotically stable $[2]$.

Under this stability condition, Bélanger derives the following result for the lagged products [ 9 ]

$$
E\left[\nu(k) v^{\top}(k-1)\right]=\sum_{i=1}^{N} f_{i}(k, 1) \alpha_{i}
$$

where the $f_{i}(k, 1)$ are given recursively by

$$
\begin{aligned}
& f_{i}(k, 0)=H(k) M_{i}(k, 0) \\
& f_{i}(k, 1)=H(k) M_{i}(k, 1) ; 1>0
\end{aligned}
$$

and the vectors $M_{i}(k, 1)$ by

$$
\begin{aligned}
& M_{i}(k, 1)=\Phi(k-1) M_{i}(k-1,1-1) ; 1>1 \\
& M_{i}(k, 1)=\Phi(k-1) M_{i}(k-1,0)-K(k-1) R_{i} \\
& M_{i}(k, 0)=S_{i}(k) H^{\top}(k) \\
& M_{i}(k, 1)=0 ; k<1
\end{aligned}
$$


The $S_{i}(k)$ scalars satisfy

$$
\begin{aligned}
& S_{i}(k+1)=\Phi(k) S_{i}(k) \Phi^{\top}(k)+K(k) R_{i} K^{\top}(k)+Q_{i} \\
& S_{i}(0)=0
\end{aligned}
$$

Next, it is desired to obtain the estimate $\hat{\alpha}(k)$. We proceed

by defining the scalar

$$
\sigma(k, 1)=\nu(k) \nu(k-1)
$$

and the row vector

$$
\left.F(k, 1)=\left[f_{1}(k, 1) f_{2}(k, 1)\right] \ldots f_{N}(k, 1)\right]
$$

Equation (5.13) may now be written as

$$
E[\sigma(k, 1)]=F(k, 1) \alpha
$$

and by defining a noise variable $\eta(k, 1)$, we may write

$$
\sigma(k, 1)=F(k, 1) \alpha+\eta(k, 1)
$$

where, clearly

$$
E[\eta(k, 1)]=0
$$

We now agree to measure the lagged products $\sigma(k, 1)$ in the order 


$$
\sigma(1,0), \sigma(1,1), \ldots, \sigma(1, L), \sigma(2,0), \ldots, \sigma(2, L), \ldots
$$

and use an integer $t$ to count these observations

$$
t=(k-1)(L+1)+1+1
$$

Equation (5.25) becomes

$$
\sigma(t)=F(t) \alpha+\eta(t)
$$

To estimate $a$, the following optimization problem is posed:

Find the vector $\hat{a}(t)$ that minimizes

$$
\begin{aligned}
J(t) & =\sum_{i=1}^{t}[\sigma(i)-F(i) a]^{\top} R(i)[\sigma(i)-F(i) \alpha] \\
& +\left(\alpha-\alpha_{0}\right)^{T} \Gamma_{0}^{-1}\left(\alpha-\alpha_{0}\right)
\end{aligned}
$$

where $R(i), i=1,2, \ldots, t$ and $\Gamma_{0}$ are positive definite matrices.

This optimization problem is amenable to the recursive solution

$$
\begin{aligned}
& \hat{\alpha}(t)=\hat{\alpha}(t-1)+\Gamma(t) F^{\top}(t) R_{0}^{-1}(t)[\sigma(t)-F(t) \hat{\alpha}(t-1)] \\
& \Gamma(t)=\Gamma(t-1)-\Gamma(t-1) F^{\top}(t)\left[F(t) \Gamma(t-1) F^{\top}(t)+\Omega(t)\right]^{-1} F(t) \Gamma(t-1)
\end{aligned}
$$

with initial conditions

$$
\hat{\alpha}(0)=\alpha_{0}, \quad \Gamma(0)=\Gamma_{0}
$$


If the following conditions hold
(1) $E[a]=x_{0}$
(2) $\left.E\left[\alpha-\alpha_{0}\right)\left(\alpha-\alpha_{0}\right)^{\top}\right]=\Gamma_{0}$
(3) $\eta(t)$ is white
(4) $R(t)=E[\eta(t) \eta(t)]$

Then $\hat{\alpha}(t)$ is the minimum-variance estimate of $a$, and $\Gamma(t)$ is the error covariance matrix. Under these conditions Equations (5.30) (5.31) represent the Kalman filter for the system

$$
\begin{aligned}
& \alpha(t)=\alpha(t-1) \\
& \sigma(t)=F(t) \alpha(t)+\eta(t)
\end{aligned}
$$

Thus we use the filter $(5.30)-(5.31)$, with $\alpha_{0}$ as the best prior estimate of $\alpha$, and a guess of the initial $\Gamma_{0} \cdot R(t)$ is generated from the Equations (see [9]),

$$
\begin{aligned}
& R(t)= \begin{cases}w(k) w(k-1) ; & 1 \neq 0 \\
2 w(k) w(k) ; & 1=0\end{cases} \\
& w(k)=F(k, 0) \hat{\alpha}(k)
\end{aligned}
$$

\subsection{Dual Control With Adaptive Filtering}

In this section we apply the adaptive filter to the dual controller 
that was developed in Chapter 3. The system is given by Equations $(3.5),(3.7)$

$$
\begin{aligned}
& x(k+1)=x(k)+\omega(k) \\
& y(k)=\varphi(k-1) x(k)+u(k)
\end{aligned}
$$

At Stage $k$

The dual controller is given by (3.49) - (3.52)

$$
\begin{aligned}
& u^{*}(k)= \begin{cases}\bar{u}(k) \text { sgn } u^{0}(k) \text { if } & \left|u^{0}(k)\right|<\bar{u}(k) \\
u^{0}(k) & \text { otherwise }\end{cases} \\
& u^{\circ}(k)=-\left[\hat{b}_{1}{ }^{2}(k+1 \mid k)\right]^{-1}\left[\hat{b}_{1}(k+1 \mid k) \hat{a}_{c}(k+1 \mid k)\right. \\
& \left.+P_{n+i}(k+1 \mid)\right]^{\top} Y(k) \\
& \bar{v}(k)= \begin{cases}\sqrt{\mu}(k) & \text { if } \mu(k)>0 \\
0 & \text { otherwise }\end{cases} \\
& \mu(k)=R\left[\bar{p}(k+1)-\operatorname{tr} P^{-1}(k+1 \mid k)\right]-Y^{\top}(k) Y(k)
\end{aligned}
$$

$\alpha_{c}(k)$ and $Y(k)$ are given by (3.12) and (3.13) respectively.

The estimate and error variances are given by the adaptive filter 


$$
\begin{aligned}
& \hat{x}(k+1 \mid k)=\hat{x}(k \mid k-1)+K(k) \nu(k) \begin{aligned}
\nu(k)= & y(k)-\varphi(k-1) \hat{x}(k \mid k-1) \\
K(k)= & P(k \mid k-1) \varphi(k-1)\left[\varphi(k-1) P(k \mid k-1) \varphi^{\top}(k-1)\right. \\
+ & \hat{R}(k)]^{-1}
\end{aligned} \\
& P(k+1 \mid k)=\Phi(k) P(k \mid k-1) \Phi^{\top}(k)+K(k) \hat{R}(k) K^{\top}(k)+\hat{Q}(k) \\
& \hat{Q}(k)=\sum_{i=1}^{N} \hat{a}_{i}(k) Q_{i} \cdot \\
& \hat{R}(k)=\sum_{i=1}^{N} \hat{a}_{i}(k) Q_{i} \\
& \hat{a}(k)=\left[\hat{a}_{1}(k) a_{2}(k) \ldots a_{N}(k)\right]^{\top} \\
& \Phi(k)=I-K(k) \varphi(k-1)
\end{aligned}
$$

with the relation

$$
t=(k-1)(L+1)+I+1 ; i=0,1,2, \ldots, L
$$

in mind, $\hat{a}(k)$ is updated according to

$$
\hat{a}(k)=\hat{a}\left[t_{k}=k(L+1)\right]
$$

$t_{k}$ is the value of $t$, where the $\hat{\alpha}$ is updated,

$$
t_{k}=(k-1)(L+1)+L+1=k(L+1)
$$


$\hat{\alpha}(t)$ is then estimated by the filter of $(5.30)$ and $(5.31)$.

Initially the filter is not updated and the lagged products are not measured, in accordance with a requirement of Bélanger's algorithm, by which, the norm of the state transition matrix of the Kalman error, must be negligibly small.

We start by fixing an initial period in which the parameters are identified by using some fixed $\alpha_{0}$, and thus the resulting $Q_{0}$ and $R_{0}$. At the end of this interval we start the adaptive filter by measuring $L+1$ innovations

$$
\nu(i)=y(i)-\varphi(i-1) \hat{x}(i \mid i-1) ; i=1,2, \ldots, L+1 .
$$

When $i=L+1$ we determine $k=1, t=1$ and start the dual-control-adaptivefiltering policy. At each stage $k$ the integer $t$ varies by $L+1$ units.

$$
\text { at } k: t=(k-1)(L+1)+1, \ldots,(k-1)(L+1)+L+1
$$

The update $\hat{a}(k)$ is done at the upper value of $t, t_{k}=(k-1)(L+1)+L+1=k(L+1)$

\subsection{System Simulation}

The system simulated is of first order with two random parameters as described in Chapter 4, Section 4.2. The observation equation is,

$$
\begin{aligned}
& y(k)=p(k-1) \times(k)+v(k) \\
& \varphi(k-1)=[y(k-1) \quad u(k-1)]
\end{aligned}
$$


The parameters are the random walk processes,

$$
\begin{aligned}
& x(k+1)=x(k)+\omega(k) \\
& x(k)=[a(k) b(k)]^{\top} \\
& \omega(k)=\left[\omega_{a}(k) \omega_{b}(k)\right]^{\top} \\
& \omega(k) \sim N[0, Q], v(k) \sim[0, R]
\end{aligned}
$$

The noise covariances $Q$ and $R$ are not known. Their actual values are

$$
\begin{aligned}
& Q=\operatorname{diag}\left[q_{a a} q_{b b}\right]=\operatorname{diag}\left[\begin{array}{ll}
0.0002 & 0.02
\end{array}\right] \\
& R=0.04
\end{aligned}
$$

The following matrices and scalars are assumed

$$
\begin{aligned}
& Q_{1}=\operatorname{diag}\left[\begin{array}{ll}
0.002 & 0
\end{array}\right] \\
& Q_{2}=\operatorname{diag}\left[\begin{array}{ll}
0 & 0.08
\end{array}\right] \\
& Q_{3}=\operatorname{diag}\left[\begin{array}{ll}
0 & 0
\end{array}\right] \\
& R_{1}=0, R_{2}=0, R_{3}=0.16
\end{aligned}
$$

The filter uses the estimated covariances

$$
\begin{aligned}
& \hat{Q}(k)=\sum_{i=1}^{3} \hat{a}_{i}(k) Q_{i} \\
& \hat{R}(k)=\sum_{i=1}^{3} \hat{a}_{i}(k) R_{i}
\end{aligned}
$$




\subsubsection{Example 1 Comparison Of Average Performance}

Four modes of control are simulated and compared as to the performance of the control policies.

The first case is that of non-dual control as given by Equation (5.38), and non-adaptive filtering. The second case consists of the dual control strategy as given by Equations $(5.37)-(5.40)$, with non-adaptive filter. In the third case we use the non-dual controller but with an adaptive filter, and finally the fourth control case is that of dual control and adaptive filtering.

In the first and second cases, where a non-adaptive filter is used, it is assumed for the vector a

$$
a_{0}=[1.1 .1 .]
$$

a constant vector. Consequently the filter and controller use the assumed noise covariances $Q_{\text {as }}$

$$
\begin{aligned}
& Q_{a s}=\operatorname{diag}\left[\begin{array}{ll}
0.002 & 0.08
\end{array}\right] \\
& R_{a s}=0.16
\end{aligned}
$$

while the actual covariances are those given by $(5.57)$ and $(5.58)$.

The value of $a_{0}$ in (5.65) is used as the initial guess of $\hat{a}(t)$ in the adaptive filter, $\hat{\alpha}(0)=\alpha_{0}$, and the noise covariances are tracked and updated in the third and fourth control cases according to (5.63) and (5.64). The initial 
error covariance matrix in the filter for $a(t)$ is taken as $\Gamma(0)=1$, the unit matrix.

The four performance curves are averaged over 45 runs, which are simulated with randomly chosen noise samples.

The initial estimates of the system parameters are taken as
â. $(t)=0.1$
$\hat{b}(1)=0.1$

and the filter initial error covariance matrix is

$$
P(1 \mid 0)=\operatorname{diag}\left[\begin{array}{ll}
4.0 & 4.0
\end{array}\right]
$$

The actual initial values of the system parameters are taken randomly from the distribution

$$
\begin{aligned}
& a(1) \sim N[0.7,0.01] \\
& b(1) \sim N[1.5,0.1]
\end{aligned}
$$

The average accumulated loss functions

$$
\bar{L}(k)=\overline{\sum_{i=1}^{K} y^{2}(i)}
$$

are given in Figure 5.1. The performance of the non-dual controller with adaptive filtering is approximately the same as that of the dual controller with non-adaptive filtering. The superior identification of the first, is partly cancelled by the nonduality of its control law, which leads to the occurence of turn-off and burst 


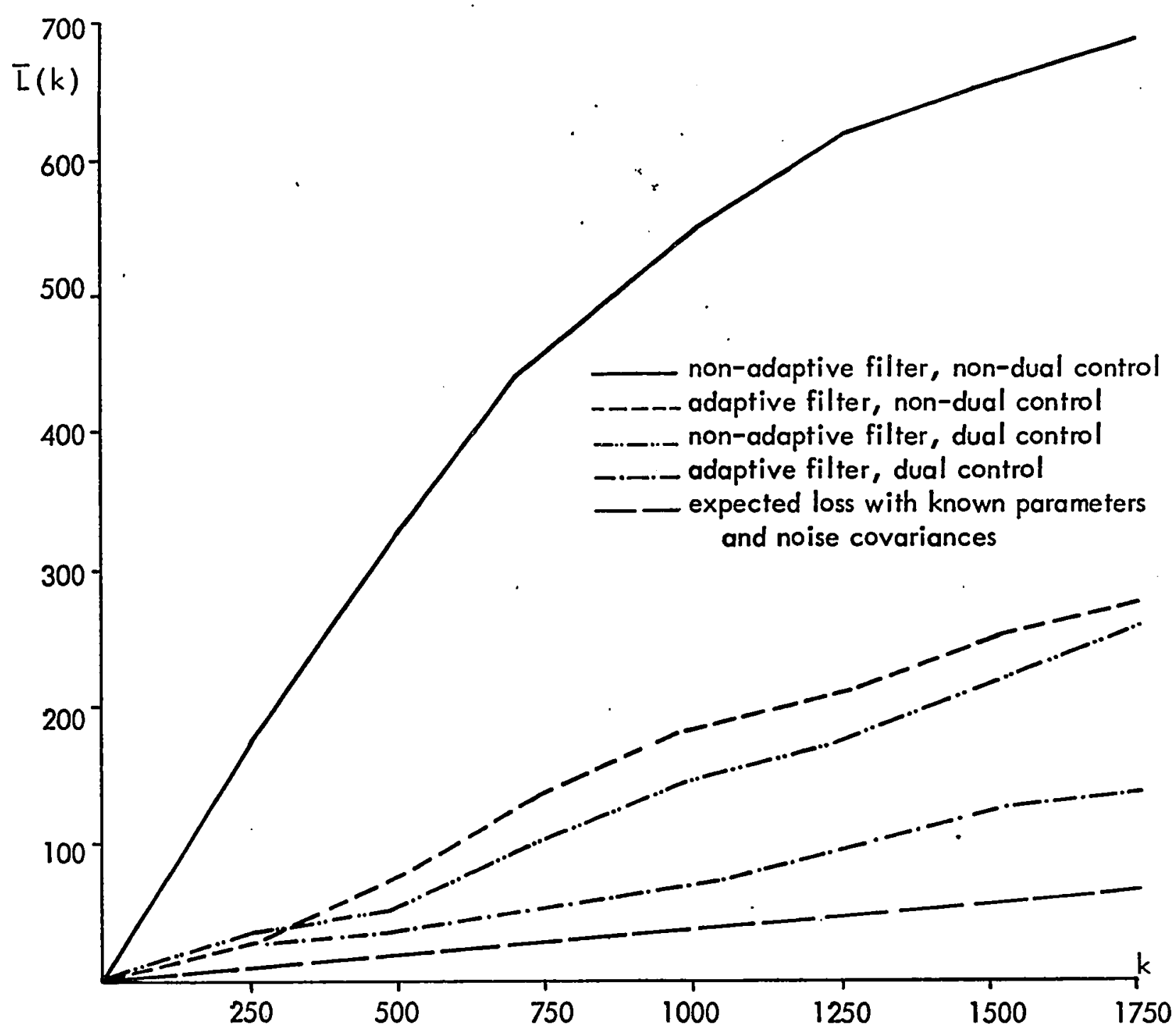

FIGURE 5.1 AVERAGE ACCUMULATED LOSS FUNCTIONS, EXAMPLE 1. 
phenomena. The advantage of the dual controller in the second case, is on the other hand offset by the inferior indentification capability of the filter which is using the wrong noise covariances.

The performance of the non-dual control with non-adaptive filter, is seen to be quite poor, while the combined dual controller and adaptive filter gives a very large and fundamental improvement.

The dual controller uses the trace constraint according to Equation (4.30) in Section 4.2, with the parameters,

$$
\rho_{c}=11 ; P_{b b}^{M}=2.5 ; a_{b}=1.2
$$

\subsubsection{Example 2 Single Run Results}

In this example we compare the performance of the four control policies, for a single run, one of those used in the previous example. In the four cases the same noise samples are used. The four control policies are:

(1) Non-dual control, non-adaptive filter.

(2) Dual control, non-adaptive filier.

(3) Non-dual control, adaptive filter.

(4) Dual control, adaptive filter.

The four accumulated loss curves are shown in Figure 5.2. In three of them there is a large jump of the loss function just after stage 900 . The cause of this jump is the burst phenomenon. The gain parameter $b(k)$ becomes small above 
stage 900 and changes its sign a number of times. Its estimation under the first 3 control strategies mentioned above, is quite poor, but the estimates are small in any case. The estimates $\hat{b}(k)$ just prior to stage 900 , were quite good and the error variance $P_{i b b}(k)$ was small. The result is that the controller (5.49) produces erroneously large signals and the burst phenomenon is on. After a relatively short period ( $50-150$ stages in our case) the filters catch on and the burst ends.

In the two cases of dual confrol we obtain the best results, especially in the fourth control policy, of dual control and adaptive filtering. The burst in this case appears very mildly, and is ended shortly due to better tracking of the parameters, resulting from the control duality coupled with the filter adaptivity.

We now proceed to observed the performance of the adaptive filter in the case of dual control with adaptive filtering. Figure 5.4 shows the estimated covariance function $\hat{q}_{\mathrm{aa}}(k)$, and Figure 5.5 describes the other covariance estimates $\hat{q}_{b b}(k)$ and $\hat{R}(k)$, as well as their actual values.

It is seen that the observation noise covariance $R$ is estimated very efficiently, as $\hat{R}(k)$ converges very fast after about 400 stages - to its final value. This final value is about 0.0355 as compared to the actual value of $R-0.04$. As was pointed out in Chapter 3 the dual adaptive control policy, is invariant under the observation noise intensity, as long as $R$ is known. The adaptive filter thus efficiently identifies this parameter.

The identification of the gain-parameter noise covariance $q_{b b}(k)$, is done less efficiently, as it is seen in Figure 5.5. The convergence of $\hat{q}_{b b}(k)$ to its 


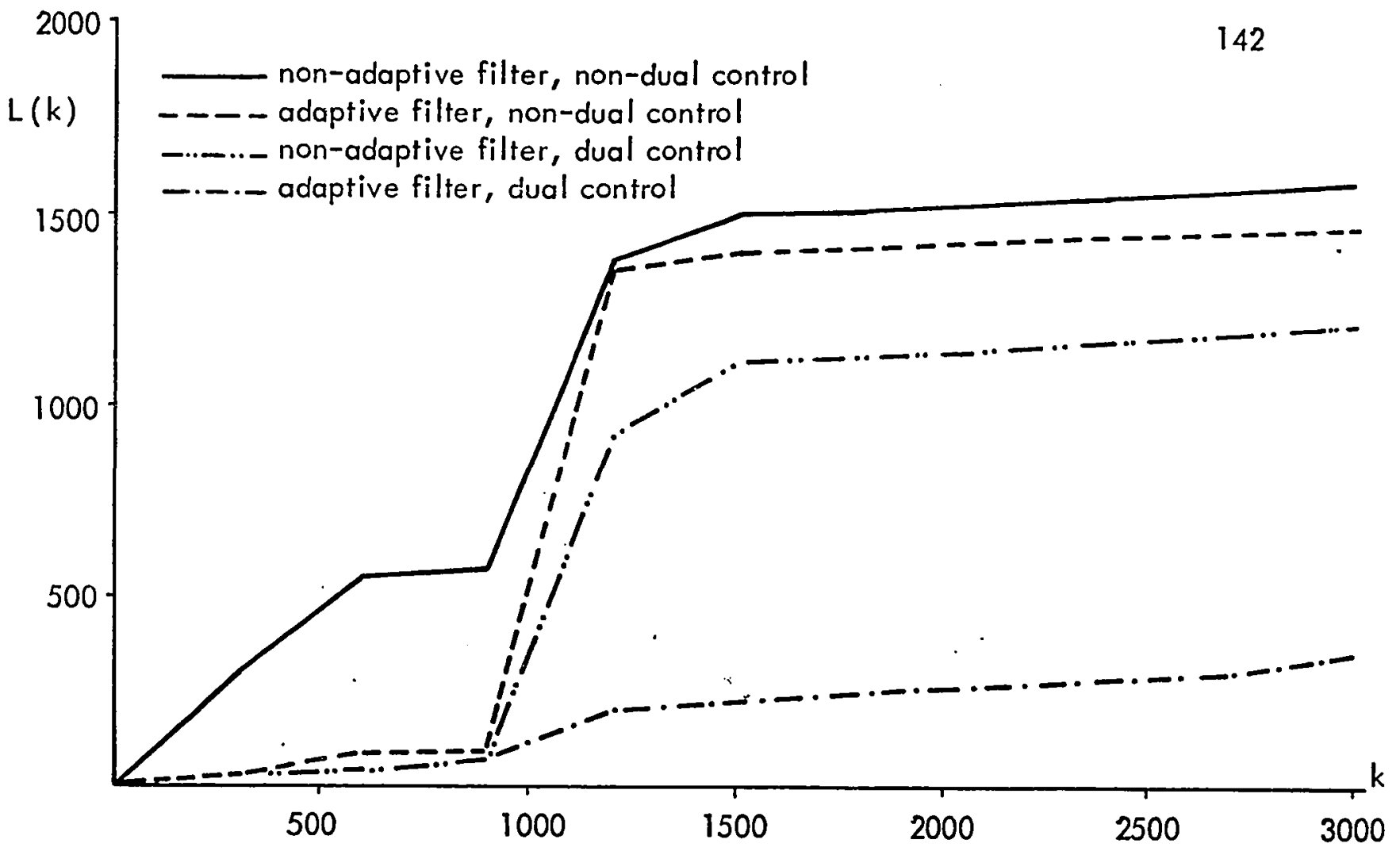

FIGURE 5.2 ACCUMULATED LOSS FUNCTIONS, EXAMPLE 2.

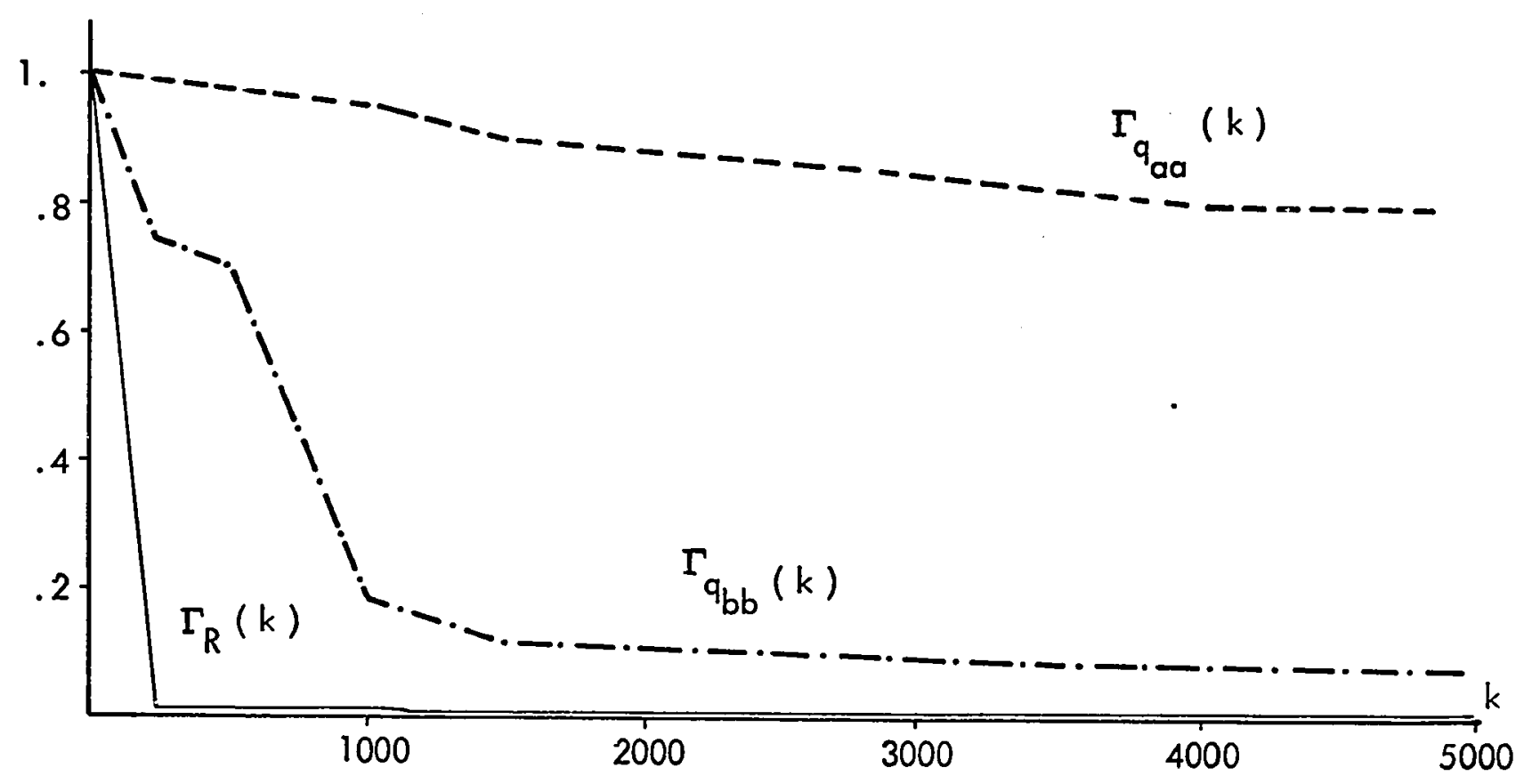

FIGURE 5.3 THE ERROR VARIANCES OF THE COVARIANCE ESTIMATES. ADAPTIVE FILTER, DUAL CONTROL, EXAMPLE 2. 


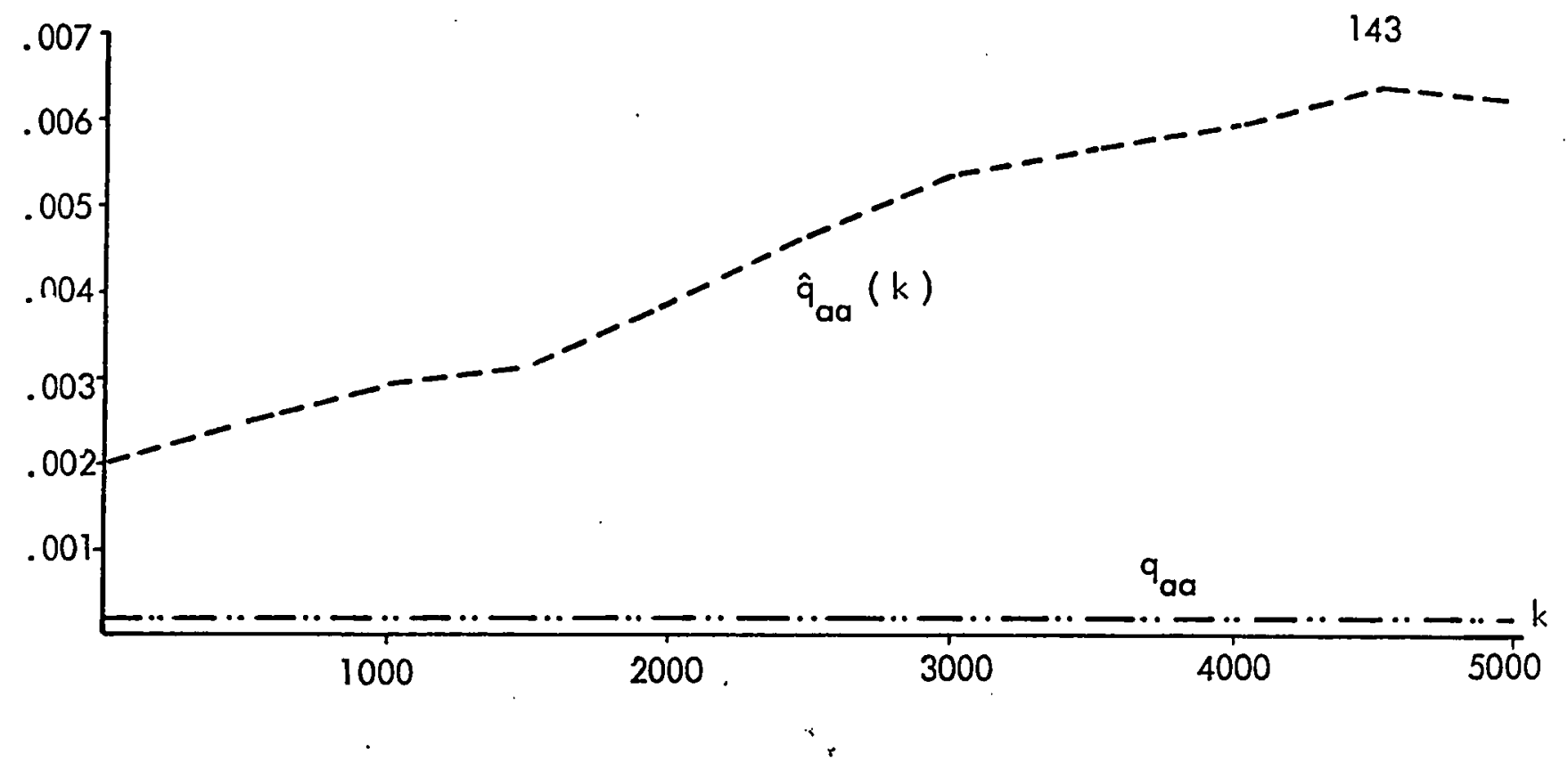

FIGURE 5.4 THE $\hat{q}_{a a}(k)$ ESTIMATE. ADAPTIVE FILTER, DUAL CONTROL, EXAMPLE 2.

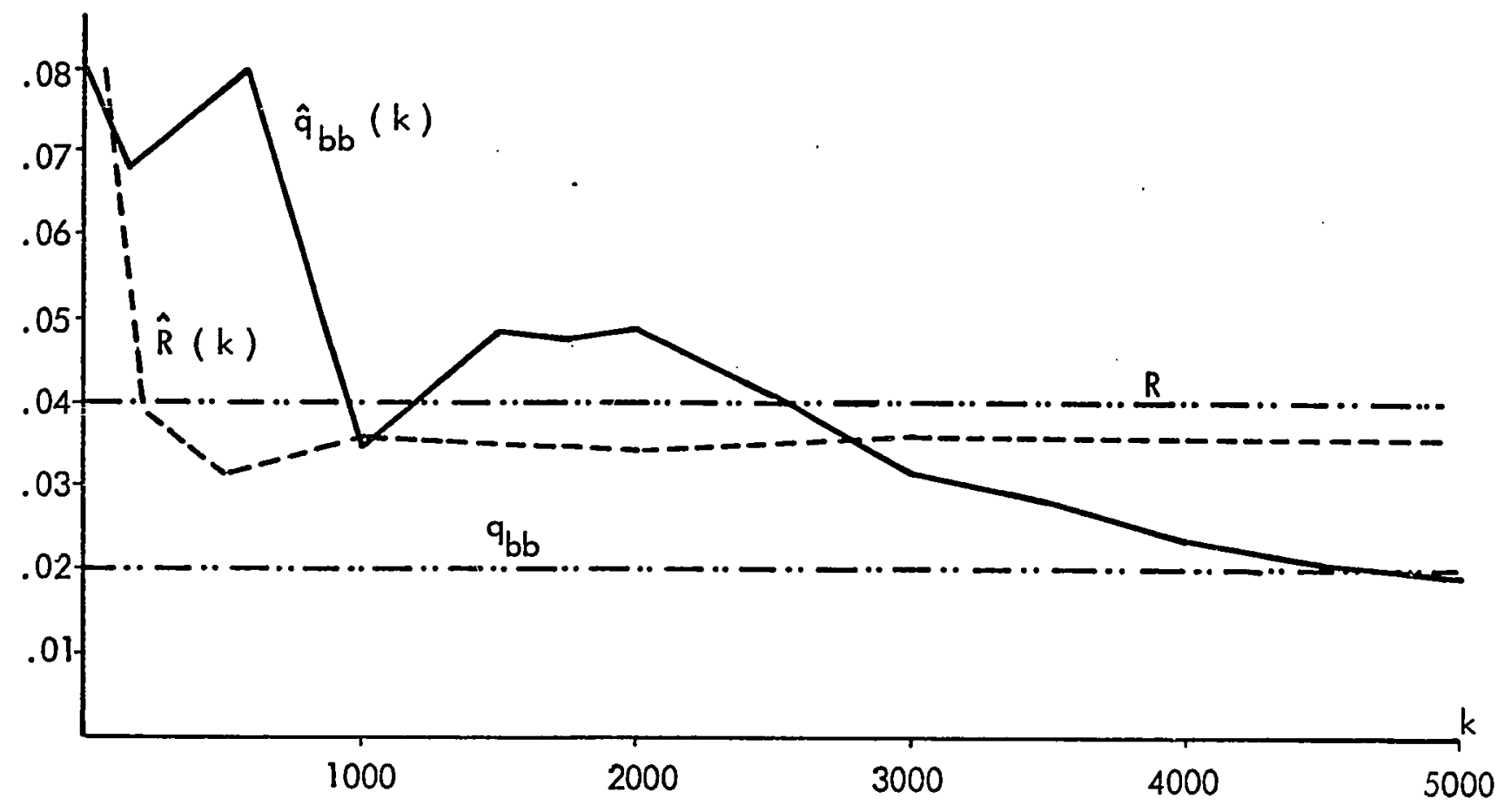

FIGURE 5.5 THE $\hat{R}(k)$ and $\hat{q}_{b b}(k)$ ESTIMATES. ADAPTIVE FILTER, DUAL CONTROL, EXAMPLE 2. 
actual value is slower than that of $\hat{R}(k)$. But the covariance filter produces a very bad estimate of the $a(k)$ parameter noise covariance. As is seen in Figure 5.5, $\hat{\mathrm{q}}_{\mathrm{bb}}(k)$, diverges from its true value. It must be noted however, that the estimate $\hat{a}(k)$ plays the least important role in the adaptive controller, and $i ;$ also assumed to vary slowly in comparison to $b(k)$. Hence its identification is quite good, despite the poor knowledge of $q_{a a}$. Even if the error in estimation $a(k)$ is large, it affects only little the controller, since the control law is independent of the error variance $p_{a a}(k)$, as can be seen from '.le control Equation (4.11).

$$
u^{0}(k)=-\frac{\hat{b}(k+i \mid k) \hat{a}(k+1 \mid k)+P_{a b}(k+1 \mid k)}{\hat{b}^{2}(k+1 \mid k)+P_{b b}(k+1 \mid k)}
$$

In Figure 5.3, one can see the error variances of the noise covariance parameter estimates $\Gamma(k)$. It is seen that $\Gamma_{R}(k)$ - the error variance of $\hat{R}(k)$ decreases fast and stays very small reflecting the good $\hat{R}(k)$ estimate. $P_{q_{b b}}(k)$ decreases also as the estimate $\hat{q}_{b b}(k)$ improves, and the variance $\Gamma_{q_{a d}}(k)$ decreases only very slowly reflecting a low confidence in $\hat{q}_{b b}(k)$. However, the fact that it decreases, points out that the estimate $\hat{q}_{a a}(k)$ will probably improve in the future or at least will not diverge further. 


\section{CHAPTER VI}

\section{CONCLUSION}

This thesis considers three main topics which are related to each other: optimal input design for identification of multivariable, steady state nonlinear systems; dual adaptive control of linear, stochastic, dynamic systems with random parameters; implementation of dual adaptive controller with adaptive filtering.

In the following we present a list of the thesis main contributions in an abbreviated form, which to the best of the author's knowledge are original. Other material in the thesis body is considered as by-products of the main contributions.

\subsection{Thesis Contributions}

1. Optimal input synthesis for steady-state systems by formulation of an optimal control problem in matrix form.

2. Development of conjugate gradients algorithm for the solution of the Two-Point-Boundary-Value-Problem with matrix equations.

3. Alternate simplified solution to the dynamic minimization problem by sequential function minimizations.

4. A technique for obtaining a dual adaptive control law, by using a measure of the system information matrix.

5. A comparison by simulations of the dual, nondual and perturbation controllers. 
6. Application of Bélanger's noise covariance estimator to the implementation of the dual adaptive control law with adaptive filtering.

\subsection{Suggestions for Further Research}

1. A further theoretical and practical investigation of the dual controller trace constraint" $p(k)$ will result in better implementation of the dual control policy.

2. The application of the theory to stochastic multivariable systems is an open field for research in the problem of adaptive dual real time controllers.

\subsection{Final Observations}

The optimal input which is designed in Chapter 2, allows best identification with relatively small number of system disturbances from its steady-state.

The dual adaptive controller is seen, by system simulations, to perform about as well as the perturbation controller when the noise statistics are known, but seems to perform better when the noise statistics are not completely known.

With incomplete knowledge of the noise covariance matrices, the dual adaptive controller in conjunction with adaptive filtering is seen to perform efficiently. 


\section{REFERENCES}

1. K.J. Aström, "Introduction to Siochastic Control Theory", Academic Press, New York, 1970.

2. A.H. Jazwinski, "Stochastic Processes and Filtering Theory", Academic Press, New York, 1970.

3. K.J. Aström and B. Wittenmark, "Problems of Identification and Control", J. Math. Anal. Appl. 10, 90-113 (1971).

4. J. Wieslander and B. Wittenmark, "An Approach to Adaptive Control Using Real Time Identification", Automatica, Vol. 7, pp. 211-217, Pergamon Press, 1971.

5. B. Wittenmark, "On Adaptive Control of Low Order Systems", Report 6918, Lund Institute of Technology, Division of Automatic Control, August 1969.

6. R.K. Mehra, "On Optimal and Suboptimal Linear Smoothing", Proc. of the National Electronics Conference, Vol. XXIV, 1968.

7. R. Nishimura, "Error Bounds of Continuous Kalman Filters and the Application to Orbit Determination Problems", IEEE Trans. Automatic Control, Vol. AC-12, No. 3, June 1967.

8. R.J. Fitzgerald, "Error Divergence in Optimal Filtering Problems", Proc. second IFAC Symp. on Automatic Control in Space, Vienne, Austria, Sept. 1967.

9. P.R. Bélanger, "Estimation of Noise Covariance Matrices for a Linear Time-Varying Stochastic Process", Preprints of the IFAC 5th world congress, Paris, France, June 1972.

10. T. Kailath, "An Innovations Approach to Least-Squares Estimation, Part 1: Linear Filtering in Additive White Noise", IEEE Trans on Automatic Control, Vol. AC-13, No. 6, December 1968. 
11. R.E. Kalman, "A New Approach to Linear Filtering and Prediction Problems", Trans. ASME, Ser.D: J. Basic Eng. 82, 35-45 (1960).

12. R.E. Kalman and R.S. Bucy, "New Results in Linear-Filtering and Prediction Theory", Trans. ASME, Ser. D: J. Basic Eng. 83, 95-108, (1961).

13. H.W. Sorenson, "Kalman Filtering Techniques", Advances in Control Systems, C.T. Leondes ed., Vol. 3, 1966.

14. H.W. Sorenson, "Controlability and Observability of Linear, Stochastic, Time-Discrete Control Systems", Advances in Control Systems, C.T. Leondes, ed., Vol. 6, 1968.

15. P.G. Kaminski, A.E. Bryson Jr. and S.F. Schmidt, "Discrete Square Root Filtering: A Survey of Current Techniques", IEEE Trans. on Automatic Control, Vol. AC-16, No. 6, December 1971.

16. A.A. Feldbaum, "Theory of Dual Control, I, II, III, IV. Automation and Remote Control No. 9, 11, 1961; No. 1, 2, 1962.

17. A.A. Feldbaum, "Optimal Control Problems", Academic Press, New York, 1965.

18. H. Halkin, "A Maximum Principle of the Pontryagin Type for Systems Described by Nonlinear Difference Equations", J. SIAM Control, Vol. 4, No. 1, 1966.

19. J.M. Holtzman, "On the Maximum Principle for Nonlinecir Discrete-Time Systems", IEEE Trans on Automatic Control, Vol. AC-11, 1966.

20. J.M. Holtzman, "Convexity and the Maximum Principle for Discrete Systems", IEEE Trans. on Automatic Control, Vol. AC-11, January 1966.

21. M. Canon, C.Cullum and E. Polak, "Constrained Minimization Problems in Finite Dimensional Spaces", J. SIAM Control, Vol. 4, No. 3, 1966. 
22. O. Gruel and L. Lapidus, "The Maximum Principle and Discrete Systems", I \& EC Fundamentals, Vol. 7, November 1968.

23. M. Athans, "The Matrix Minimum Principle", Information and Control 11, 592-606 (1967).

24. M. Athans and F.C. Schweppe, "On Optimal Waveforms Design Via Control Theoretic Concepts", Information and Control 10, 335-337, (1967).

25. L.S. Lasdon, S.K. Mitter and A.D. Warren, "The Conjugate Gradient Method for Optimal Control Problems", IEEE Trans. on Automatic Control, Vol. AC-i2, Aprii 1967.

26. J.F. Sinnott Jr, and D.G. Luenberger, "Solution of Optimal Control Problems by the Method of Conjugate Gradients", Preprints JACC, 1967.

27. B. Pagurek and C.M. Woodside, "The Conjuge ie Gradient Method for Optimal Control Problems with Bounded Control Variables", Automatica, Vol. 4, pp. 337-349, 1968.

28. R.K. Mehra and A.E. Bryson Jr., "Conjugate Gradient Methods With an Application to V/STOLFlight-Path Optimization", J. Aircraft, Vol. 6, No. 2, 1969.

29. R. Fletcher and M. Reeves, "Function Minimization by Conjugate Gradients", Compuier Journal, July 1964.

30. J.D. Pearson, "Variable Metric Methods of Minimization", Computer Journal, May 1969.

31. L. Meier, J. Peschon and R.M. Dressler, "Optimal Control of Measurement Subsystems", Preprints JACC, 1967.

32. G.R. Tait, "The Array-Matrix Concept - A New Approach to Multivariate Analysis", Ph.D. Thesis, Department o: Electrical Engineering, McGill University, March 1971. 
33. R.E. Kalman, "Contributions to the Theory of Optimal Control", Bol. Soc. Mat. Mexicana 5, 102-119 (1960).

34. R.E. Kalman, "On the General Theory of Control Systems", Proc. IFAC Congress Ist 1, 481-491 (1961).

35. N.E. Nahi, "Estimation Theory and Applications", Wiley, New York, 1969.

36. N.E. Nahi and D.E. Wallis Jr., "Optimal Inputs for Parameter Estimation in Dynamic Systems with White Observation Noise", Proc. JACC, 1969.

37. F.C. Schweppe, "On the Accuracy and Resolution of Radar Signals, "IEEE Trans. on Aerospace and Electronic Systems", Vol. AES-1, No. 3, 1965.

38. S. Kullback, "Information Theory and Statistics", Wiley, New York, 1959.

39. $\quad$ S.S. Wilks, "Mathematical Statistics" , Wiley, New York, 1962.

40. H. Cramer, "Mathematical Methods of Statistics", Princeton University Press, Princeton, N.J., 1946.

41. M. Athans and P.L. Falb, "Optimal Control", McGraw-Hill, 1966.

42. A.P. Sage, "Optimum Systems Control", Prentice-Hall, Englewood Cliffs, N.J., 1968.

43. A.P. Sage and J.L. Melsa, "Estimation Theory with Applications to Communications and Control", McGraw-Hill, New York, 1971.

44. A.E. Bryson Jr., and Y.C. Ho, "Applied Optimal Control", Ginn and Comp., Waltham, Mass., 1969.

45. M. Aoki and R.M. Staley, "On Input Signal Synthesis in Parameter Identification", Automatica 6, 431-440 (1969).

46. M. Aoki and R.M. Staley, "Some Computational Considerations in Input Signal Synthesis Problems", Computing Methods in Optimization Problems-Vol. 2 (Eds. Zadeh, Neustadt, Balakrishnen). Academic Press, New York, 1969. 
47. V.S. Levadi, "Design of Input Signals for Parameter Estimation", IEEE Trans. Aut. Control AC-11, 205-211, 1966.

48. D.R. Vander Stoep, "Trajectory Shaping for Minimization of State Variable Errors", IEEE Trans. Aut. Control., June 1968.

49. W.J. Murphy, "Optimal Stochastic Control of Discrete Linear Systems With Unknown Gain", IEEE Trans. Aut. Control, August 1968.

50. K.J. Aström and P. Eyhhoff, "System Identification - A Survey", Automatica 7, 123-162 (1971).

51. J.B. Farison, R.E. Graham and R.C. Shelton, "Identification and Control of Linear Discrete Systems", IEEE Trans. Aut. Control 12 (1967).

52. D.D. Sworder, "A Study of the Relationship Between Identification and Optimization in Adaptive Control Problems", J. Franklin Inst. 281, 198-213 (1966).

53. A.D. Sage and G.W. Husa, "Adaptive Filtering With Unknown Prior Statistics", Preprints JACC, 1969.

54. R.K. Mehra, "On the Identification of Variances and Adaptive Kalman Filtering", IEEE Trans. Aut. Control. Vol. AC-15, No. 2 April 1970.

55. P.D. Abramson, Jr., "Simultaneous Estimation of the State and Noise Statistics in Linear Dynamic Systems, NASA Technical Report, NASA TR R - 332, March 1970.

56. A.H. Jazwinski, "Adaptive Filtering", Automatica Vol. 5, 475-485 (1969).

57. W.M. Wonham, "Optimal Stochastic Control", Automatica, Vol. 5, 113-118 (1969). 


\section{APPENDIX A}

We show here that the convexity $[18]$ or directional convexity [19] assumptions, needed for the application of the discrete minimum principle, do not hold for the discrete optimal control problem of (2.31) - (2.32).

Regarding the dynamic constraint Equation (2.29) which is rewritten as

$$
X(k+1)-X(k)=F_{k}\left(X_{r}(k), u(k)\right)
$$

with

$$
u(\dot{k}) \epsilon \Omega=\{u(k):|u(k)| \leq \rho\},
$$

the convexity property states that the sets

$$
V(k, u)=\left\{F_{k}(X(k), u(k): u(k) \in \Omega\}\right.
$$

are convex for any $X(k)$ and $k=i, \ldots, N$.

We recall that the set $V(k, u)$ is convex if every pair of elements $x, y \in V(k, u)$ implies

$$
\alpha x+(1-\alpha) y \in V(k, u), \quad 0<a<1 \text {. }
$$

The next example shows that the convexity assumption does not hold in our case. Consider the measurement system

$$
y(k)=u^{2}(k) x_{1}(k)+u(k) x_{2}(k)+v(k)
$$


where $y(k)$ and $u(k)$ are scalars. Thus

$$
\begin{aligned}
& y(k)=C(u(k)) x(k)+v(k) \\
& C(u(k))=\left[\begin{array}{ll}
u^{2}(k) & u(k)
\end{array}\right] ; x(k)=\left[x_{1}(k) x_{2}(k)\right]^{\top}
\end{aligned}
$$

To evaluate $F_{k}(X(k), u(k))$ we take $X(k)=I, Q(k)=0$, and obtain from Equation (2.29)

$$
F(u)=-\frac{1}{u^{4}+u^{2}+R}\left[\begin{array}{cc}
u^{4} & u^{3} \\
u^{3} & u^{2}
\end{array}\right]
$$

where we use $u$ and $R$ for $u(k)$ and $R(k)$, and $k$ is fixed. We show that the set

$$
V(u)=\{F(u):|u| \leq \rho\}
$$

is not convex.

$$
\text { Take } \begin{aligned}
\alpha=\frac{1}{2}, u_{1} & =\eta, u_{2}=-\eta \text { such that }|\eta| \leq \rho \text { and } \eta \neq 0 \text {. Then } \\
& \alpha \mathrm{F}\left(u_{1}\right)+(1-\alpha) F\left(u_{2}\right) \\
& =-\frac{1}{2} \frac{1}{\eta^{4}+\eta^{2}+R}\left[\begin{array}{cc}
\eta^{4} & \eta^{3} \\
\eta^{3} & \eta^{2}
\end{array}\right]-\frac{1}{2} \frac{1}{\eta^{4}+\eta^{2}+R}\left[\begin{array}{cc}
\eta^{4} & -\eta^{3} \\
-\eta^{3} & \eta^{2}
\end{array}\right] \\
& =-\frac{1}{\eta^{4}+\eta^{2}+R}\left[\begin{array}{cc}
\eta^{4} & 0 \\
0 & \eta^{2}
\end{array}\right] \& V(u)
\end{aligned}
$$


Since no $u$ exists such that $u^{2}=0$ and $u^{3}=0$. The set $V(u)$ is thus not convex. 


\section{APPENDIX B}

Theorem 2.1 is extended here to the case where the parameters are modeled by stochastic processes. The system model is

$$
\begin{aligned}
& x(k+1)=x(k)+w(k) \\
& y(k)=C(k) x(k)+v(k) ; C(k)=C(u(k)) \\
& w(k) \sim N[0, Q(k)] \\
& v(k) \sim N[0, R(k)]
\end{aligned}
$$

Theorem 2.1 applies to the parameter model (B. I) as well. The proof is exactly the same as that of Theorem 2.1, but the notation is more cumbersome here. The covariance matrix Equation (2.29) is

$$
X(k+1)=X(k)+X(k) C^{\top}(k)\left[C(k) X(k) C^{\top}(k)+R(k)\right]^{-1} C(k) X(k)+Q(k)
$$

which, after applying lemma 2.1 becomes

$$
X(k+1)=\left[X^{-1}(k)+C^{\top}(k) R^{-1}(k) C(k)\right]^{-1}+Q(k)
$$

and recursively we may write (using $R$ and $Q$ for $R(k)$ and $Q(k)$, for simplicity),

$$
\begin{aligned}
X(N+1)= & {\left[\left[\ldots\left[X^{-1}(1)+C^{\top}(1) R C(1)\right]^{-1}+Q\right]^{-1}+C^{\top}(2) R^{-1} C(2)\right]^{-1} } \\
& \left.\left.\left.+Q]^{-1}+\ldots+Q\right]^{-1}+C^{\top}(i) R^{-1} C(i)\right]^{-1}+Q\right]^{-1}+\ldots \\
& \left.\left.\left.\left.+C^{\top}(i+1) R^{-1} C(i+1)\right]^{-1}+Q\right]^{-1}+\ldots+C^{\top}(N) R^{-1} C(N)\right]^{-1}+Q\right]^{-1}
\end{aligned}
$$


where $C(i)=C(u(i)), i=1, \ldots, N$.

The dynamic $\mathrm{N}$-stage optimization problem is

$$
\min _{u(1), \ldots, u(N)} \operatorname{tr} X(n+1)
$$

where $\operatorname{tr} X(n+1)$ is again a symmetric function of all the stage vectors $u(i)$ and by the same proof of theorem 2.1 it is seen that (B.8) can be done sequentially by $N$ single stage minimizations. 